\title{
Copyright
}

by

Felicia Angélica Durán

2010 
The Dissertation Committee for Felicia Angélica Durán Certifies that this is the approved version of the following dissertation:

\section{Probabilistic Basis and Assessment Methodology for Effectiveness of Protecting Nuclear Materials}

Committee:

Sheldon Landsberger, Co-Supervisor

Gregory D. Wyss, Co-Supervisor

Steven Biegalski

Erich Schneider

Randall Charbeneau 


\title{
Probabilistic Basis and Assessment Methodology for Effectiveness of Protecting Nuclear Materials
}

by

Felicia Angélica Durán, B.S.M.S.E.; M.S.

\author{
Dissertation \\ Presented to the Faculty of the Graduate School of \\ The University of Texas at Austin \\ in Partial Fulfillment \\ of the Requirements \\ for the Degree of \\ Doctor of Philosophy
}

The University of Texas at Austin

December 2010 


\section{Dedication}

With deep love and gratitude, I dedicate this endeavor to my family who love me, teach me, encourage me, believe in me and inspire me - especially to Mary Jane and Amelia Jane, and to honor the memories of Cruzita, José Dolores, Sarah, David, and Rudolfo 


\section{Acknowledgements}

In this endeavor, I am thankful to have had the opportunity to complete the majority my $\mathrm{PhD}$ studies through the Sandia National Laboratories* (Sandia) Doctoral Studies Program (DSP). Once upon a time, when I was developing a love of school, my father told me that if I studied hard and kept getting good grades, I could get paid for going to school. A few decades later in life, it actually did happen, and for most of the time I've been working on my $\mathrm{PhD}$, I have had the tremendous privilege of having as my "job" an activity that I have always most enjoyed - going to school. My participation in the DSP was supported by my managers and the University Programs staff at Sandia. My thanks go especially to Robert Waters and Mark Allen for their support at the start, to Carla Ulibarrí for her support at the finish, and to Bernadette Montaño, Irene Allen, and Charline Wells for their support in University Programs.

I am most appreciative of my advisors, Greg Wyss and Professor Sheldon Landsberger. To Greg, who is also a long-time colleague and a good friend, I give my deepest, heartfelt thanks for his constant, patient, impressively knowledgeable, and at times exuberant guidance and support. I consider it my great fortune to work with him and am grateful for all that I have learned from him. I would like to thank Professor

\footnotetext{
* Sandia National Laboratories is a multi program laboratory operated by Sandia Corporation, a wholly owned subsidiary of Lockheed Martin Corporation, for the U.S. Department of Energy's National Nuclear Security Administration under Contract DE-AC04-94-AL850000.
} 
Sheldon Landsberger for his experience and abilities as a university educator and his leadership of the distance education program in Nuclear and Radiation Engineering at The University of Texas at Austin. I would also like to thank the members of my $\mathrm{PhD}$ committee, Professors Steven Biegalski, Erich Schneider, and Robert Charbeneau, for their thoughtful review of this technical work.

I owe a special thanks to my current manager, Carla Ulibarrí who has been unfailing in her support and encouragement of my efforts to finish this work and of Greg's efforts as my research advisor. I wish to acknowledge her technical expertise and skilled leadership as manager of the Security Systems Analysis Department at Sandia. Most importantly, I appreciate her attentive ear to both problems and successes and her caring and firm words of wisdom when I need them.

This research was supported by Sandia in part by the Laboratory Directed Research and Development Program, the DSP, and Tuition Assistance. My colleagues at Sandia are a tremendous source of technical and scientific knowledge. I want to especially acknowledge Pamela Dawson for sharing her work on insider analysis, material control and accountability (MC\&A), and material assurance indicators and for her support at the outset of this work; John Darby for the much-needed instruction on probabilistic convolution; and Ben Cipiti for his collaboration on possible application of these methods for advanced fuel cycle facilities. Manuel Trujillo, Michael Benson, José Rodriguez, and Bruce Berry provided technical expertise and collaboration in the areas of MC\&A and analysis of insider theft. I appreciate the opportunity to work with Greg Conrad, Bruce Held, David Duggan, and Steve Conrad on other research related to the insider threat. Also at Sandia, I have to thank three wonderful women who, in the course of my research, I have dubbed the "Library Ladies." Betsy Krause, Sandy Halliday, and Kathleen Lee of the Building 823 Library provided an abundance of assistance to me, 
doing more than a few literature searches, locating copious and of course vital references, and taking care of my numerous renewals. They were never wary when they saw me coming; in fact, they always seemed pleased to see me and ready to go forth and seek out the latest critical reference. I admire their talents as professional librarians. I also thank Diane Ross, a most accomplished technical editor, for her review of the final draft of this dissertation, and Gregg Flores and Joy Lynn Giron, co-workers who provide tremendous support and friendship. Brad Key from QinetiQ, North America, provided instruction on and support for two software programs: Analytic System and Software for Evaluating Safeguards and Security (ASSESS) and Adversary Time-Line Analysis System (ATLAS). Brad is always willing to discuss and answer questions about any aspect of the insider problem and is most knowledgeable about ATLAS and ASSESS. I also wish to acknowledge the interest and support of Jeff Jay, Scott Boeke, and Tom Williams at the Savannah River Site, and Richard Donovan and Debarah Holmer at the U.S. Department of Energy to consider this work for possible operational and policy applications.

So many friends and colleagues have provided a constant source of encouragement and support during the years that I have been working toward this accomplishment. Vince Dandini and Beth Richards pursued their PhD degrees midcareer as I did, and I appreciate their examples and the many conversations we shared about the challenges of this type of endeavor. I know they truly understand the experience I've had in this endeavor. I appreciate the opportunities I've had to work with and learn from Allen Camp, John Kelly, Tim Wheeler, Frank Wyant, Donnie Whitehead, and Jeff LaChance. My friends in World Senior Racquetball, Paula Sperling, Gary Mazaroff, Luke St. Onge, Ralph and Edwina Waddington, Joe and Carol Gellman, Jane and Jerry Bentley, knew me long before I started this work and have always encouraged 
and supported me. Suzette Driggers, Doctor of Oriental Medicine and treasured friend, provided immeasurable skill and care to restore and sustain my health and wellness throughout this journey. Gina Stack provided expertise in a legal matter that had to be dealt with in the months when I was trying to finish this work. Her guidance allowed me to minimize the distraction this matter had on finishing this work, and I am happy to now have her as a valued friend. Warren and Anne Von Worley and Ed and Phoebe Tate are wonderful, caring, and encouraging neighbors who on a regular basis inquired on the progress of my work.

My most important acknowledgements are to my family to whom I have dedicated this most significant effort of my career to date. My parents taught me, among so many of the things I think I know now, the importance and value of education. My mother, a wonderful woman who has always supported her children and now her grandchildren in all their endeavors, is also the best example of a loving and caring daughter, sister, mother, grandmother, aunt, and friend. My 14-year-old daughter, by far the most amazing and joyful part of my life, is growing into an accomplished young woman, in spite of the too many times over the last few years when I had to say, "Not right now, sweetheart, I have to work on my PhD." She's smart, strong, caring, athletic, and funny, and I love her more than words can say. My daughter's paternal grandparents are a constant source of love, encouragement and support for both me and my daughter. My siblings are also among my best friends. I honor the memories of my grandparents and uncles and treasure the experiences I've shared with them and my parents, siblings, and many aunts, uncles, cousins, nephews and nieces who have been my most important teachers and have fostered my love of learning. I hope I will always live up to their regard of me and my abilities. 
Finally, I wish to acknowledge the most important thing I have learned in completing this work - that this type of endeavor seemingly undertaken by an individual is in reality supported by a cast of many. In the final months of completing this work, it is with a sense of amazement and deep gratitude that I look back on this experience, so very thankful for the love and support of family and friends, for the people I've met, worked with and learned from along the way, for the challenges I had to face, for the talent and abilities I was able to bring with me and the new ones I was able to develop. I am simply grateful that I am the person who had the opportunity to do this work and to share the experience with all those I've acknowledged here. 


\title{
Probabilistic Basis and Assessment Methodology for Effectiveness of Protecting Nuclear Materials
}

\author{
Publication No. \\ Felicia Angélica Durán, $\mathrm{PhD}$ \\ The University of Texas at Austin, 2010 \\ Supervisors: Sheldon Landsberger and Gregory D. Wyss
}

\begin{abstract}
Safeguards and security (S\&S) systems for nuclear facilities include material control and accounting (MC\&A) and a physical protection system (PPS) to protect nuclear materials from theft, sabotage and other malevolent human acts. The PPS for a facility is evaluated using probabilistic analysis of adversary paths on the basis of detection, delay, and response timelines to determine timely detection. The path analysis methodology focuses on systematic, quantitative evaluation of the physical protection component for potential external threats, and often calculates the probability that the PPS is effective $\left(\mathrm{P}_{\mathrm{E}}\right)$ in defeating an adversary who uses that attack path. By monitoring and tracking critical materials, MC\&A activities provide additional protection against inside adversaries, but have been difficult to characterize in ways that are compatible with the existing path analysis methods that are used to systematically evaluate the effectiveness of a site's protection system. This research describes and demonstrates a new method to incorporate MC\&A protection elements explicitly within the existing probabilistic path analysis methodology. MC\&A activities, from monitoring to inventory measurements,
\end{abstract}


provide many, often recurring opportunities to determine the status of critical items, including detection of missing materials. Human reliability analysis methods are applied to determine human error probabilities to characterize the detection capabilities of MC\&A activities. An object-based state machine paradigm was developed to characterize the path elements of an insider theft scenario as a race against MC\&A activities that can move a facility from a normal state to a heightened alert state having additional detection opportunities. This paradigm is coupled with nuclear power plant probabilistic risk assessment techniques to incorporate the evaluation of MC\&A activities in the existing path analysis methodology. Event sequence diagrams describe insider paths through the PPS and also incorporate MC\&A activities as path elements. This work establishes a probabilistic basis for incorporating MC\&A activities explicitly within the existing path analysis methodology to extend it to address insider threats. The analysis results for this new method provide an integrated effectiveness measure for a safeguards and security system that addresses threats from both outside and inside adversaries. 


\section{Table of Contents}

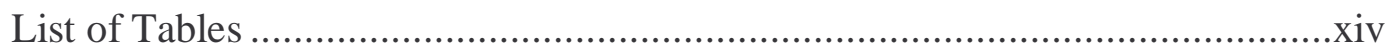

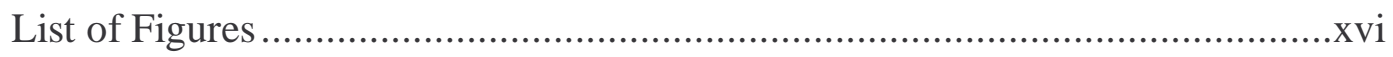

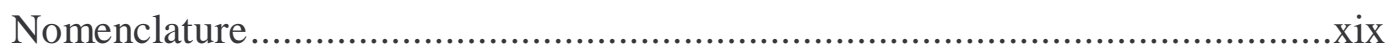

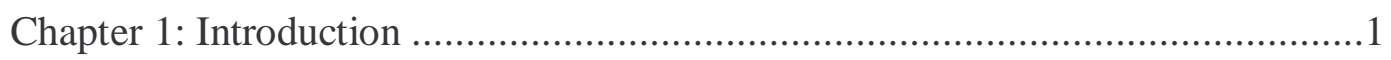

1.1 Material Control and Accounting Protection System ............................2

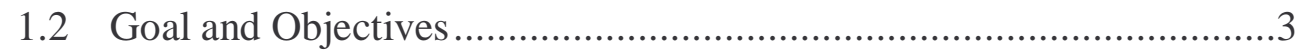

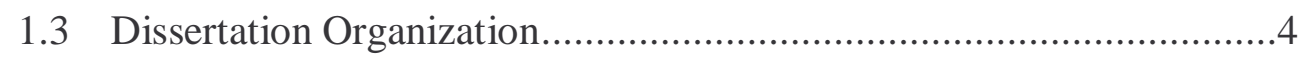

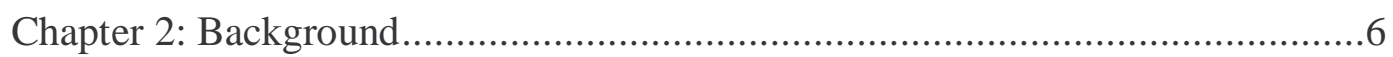

2.1 Safeguards and Security System Assessment Methodology .................6

2.2 Design and Evaluation of A Physical Protection System ....................10

2.3 Insider Studies and Evaluation of Insider Threats...............................16

2.4 Risk Assessment Tools .................................................................22

Chapter 3: Methods for Extended Path Analysis - One Daily MC\&A

Activity in One Physical Protection Layer ...................................................27

3.1 Object-Based Paradigm for Insider Theft .......................................28

3.2 Incorporating an Assessment of MC\&A Activities...............................31

3.3 Insider Theft and MC\&A Detection Timelines....................................33

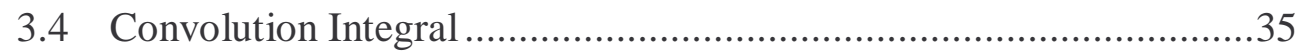

3.5 Human Reliability Models for MC\&A Activities ...............................36

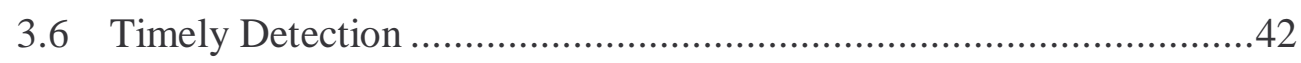

3.7 Summary of Methods Development for a Single Physical Protection Layer .................................................................................

Chapter 4: Methods for Extended Path Analysis - Daily and Combined

MC\&A Detection and Multiple Physical Protection Layers .........................53

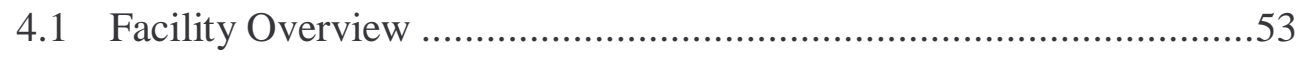

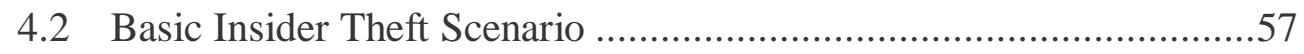

4.3 Scenarios for One Daily MC\&A Detection Activity and Varying

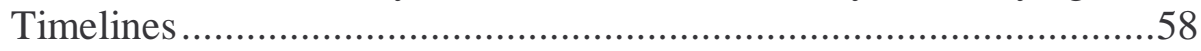


4.4 Summary of Methods Development for Daily and Combined MC\&A Detection and Multiple Physical Protection Layers...............94

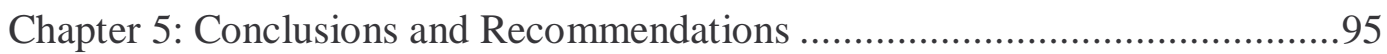

Appendix A: Combining Probability Distributions, Analytic, or Continuous

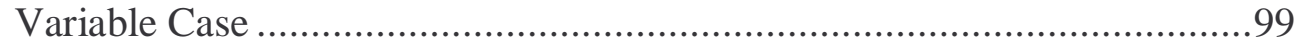

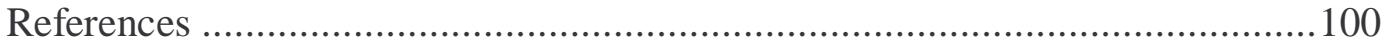

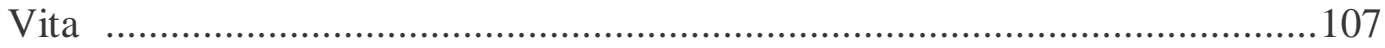




\section{List of Tables}

Table 1. Characterization of MC\&A activities as different types of NPP checking operations with estimated probabilities (HEPs) that a checker will fail to detect an error (columns 2 and 3 from [64, Table 19-1]).

Table 2. Frequencies of key administrative MC\&A activities (representative)

Table 3. Calculation of timely detection over a 30-day scenario for a uniform insider theft timeline and one MC\&A activity performed once a day based on an initial probability of detection of 0.02 , for a moderate level of dependence 46

Table 4. 30-day cumulative probability of MC\&A detection for five dependence levels for low (0.20), medium (0.50), and high (0.99) initial probability of detect

Table 5. Five scenario timelines.

Table 6. Event 4 probability of timely MC\&A detection for the five scenario timelines for five dependence levels and low (0.02), medium (0.50), and high (0.99) initial probability of detection.......74

Table 7. Event 4 probability of timely MC\&A detection for the five scenario timelines for five dependence levels and low (0.02), medium (0.50), and high (0.99) initial probability of detection .......76

Table 8: $\quad$ End state summary results for Timeline 1 - 5-day MAA/30-day PA timeline. 77

Table 9: $\quad$ End state summary results for Timeline 2 - 30-day MAA/30day PA timeline

Table 10: $\quad$ End state summary results for Timeline 3 - 90-day MAA/30day PA timeline 78

Table 11: End state summary results for Timeline 4 - 5-day MAA/5-day fixed PA timeline 78

Table 12: End state summary results for Timeline 5 - 5-day MAA/30-day fixed PA timeline. 79

Table 13: Detection timeline for a notional set of six MC\&A activities 82

Table 14: Timely MC\&A detection in the MAA (Event 2) and the PA (Event 4) for a set of MC\&A activities and geometric distributions for theft timeline 88 
Table 15: Comparison of end state summary results for 5-day MAA/30day PA timeline and geometric distributions for theft timeline for a set of MC\&A activities

Table 16: Detection timeline for set of combined MC\&A activities with a malicious insider performing activities 1 and 4 .

Table 17: Detection timeline for set of combined MC\&A activities with a malicious insider performing activity 1 and mitigation of a malicious insider performing activity 4 ................................. 92

Table 18: Timely MC\&A detection in the MAA (Event 2) and the PA (Event 4) and end state summary for baseline set of MC\&A activities, malicious insider activity and insider mitigation............94 


\section{List of Figures}

Figure 1. The system assessment methodology used by the U.S. DOE for the design and evaluation of $S \& S$ protection systems [8].

Figure 2. Design and evaluation process outline for physical protection systems [17].

Figure 3. Representation of an example facility's physical areas and possible adversary paths to a target [17]

Figure 4. Basic adversary sequence diagram for a facility [17]......................13

Figure 5. Example adversary event timeline [32].......................................15

Figure 6. Categories of malevolent insiders. ...................................................18

Figure 7a. State transition diagram for Insider Theft Object..............................29

Figure 7b. State transition diagram for Facility Object......................................29

Figure 8. Traditional event tree model of insider theft through protection

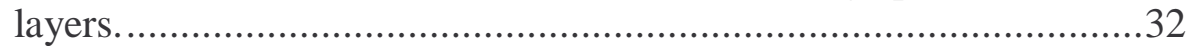

Figure 9. Insider theft modeled as an event sequence diagram (ESD) incorporating MC\&A.

Figure 10: Daily probability of detection over a 30-day period for one MC\&A activity performed once a day based on a BHEP of 0.98 , or an initial probability of detection of 0.02 , for five different levels of dependence.

Figure 11: Daily probability of detection over a 30-day period for one MC\&A activity performed once a day based on a BHEP of 0.50 , or an initial probability of detection of 0.50 , for five different levels of dependence.

Figure 12: Daily probability of detection over a 30-day period for one MC\&A activity performed once a day based on a BHEP of 0.01 , or an initial probability of detection of 0.99 , for the five different levels of dependence.

Figure 13: Daily probability of timely detection over a 30-day scenario for one MC\&A activity performed once a day based on a BHEP of 0.98 , or a 0.02 initial probability of detection, for five different levels of dependence.

Figure 14: Cumulative probability of timely detection over a 30-day scenario for one MC\&A activity performed once a day based on a BHEP of 0.98 , or a 0.02 initial probability of detection, for five different levels of dependence. 
Figure 15: Daily probability of timely detection over a 30-day scenario for one MC\&A activity performed once a day based on a BHEP of 0.50 , or a 0.50 initial probability of detection, for five different levels of dependence.

Figure 16: Cumulative probability of timely detection over a 30-day scenario for one MC\&A activity performed once a day based on a BHEP of 0.50 , or a 0.50 initial probability of detection, for five different levels of dependence.

Figure 17: Daily probability of timely detection over a 30-day scenario for one MC\&A activity performed once a day based on a BHEP of 0.01 , or a 0.99 initial probability of detection, for five different levels of dependence.

Figure 18: Cumulative probability of timely detection over a 30-day scenario for one MC\&A activity performed once a day based on a BHEP of 0.01 , or a 0.99 initial probability of detection, for five different levels of dependence.

Figure 19: Overview of Nuclear Manufacturing Facility layout [28]................54

Figure 20. ESD for tracking theft and detection.

Figure 21: Event 1 of the ESD - Detection of the insider taking the material by the PPS in the MAA

Figure 22: The ESD through Event 2 - Timely MC\&A detection in the MAA..60

Figure 23: The ESD through Event 3 - Detection of the insider moving the material by the PPS in the PA ...............................................62

Figure 24: Event tree for MC\&A events in the composite timeline. .................64

Figure 25: The ESD through Event 4 - Timely MC\&A detection in the PA......65

Figure 26: The ESD through Event 5 - Detection by the PPS of the insider moving the material out of the PA

Figure 27: Event sequence calculations for the 30-day MAA/30-day PA timeline scenarios for 0.02 initial probability of MC\&A detection and five levels of dependence.

Figure 28: Event sequence calculations for the 30-day MAA/30-day PA timeline scenarios for 0.50 initial probability of MC\&A detection and five levels of dependence.

Figure 29: Event sequence calculations for the 30-day MAA/30-day PA timeline scenarios for 0.99 initial probability of MC\&A detection and five levels of dependence.

Figure 30: Plot of material recovered + alert end state summary results for the three uniform composite timelines 
Figure 31: Plot of material recovered + alert end state summary results for the three 5-day MAA timelines and respective uniform and fixed PA timelines....

Figure 32: Daily probability of detection over a 35-day period for a set of MC\&A activities 85

Figure 33: Geometric distributions for theft timeline generated from LHS........87

Figure 34: Detection timelines for baseline set of MC\&A activities, malicious insider, and insider mitigation...................................93 


\section{Nomenclature}

\begin{tabular}{|c|c|}
\hline ASD & adversary sequence diagram \\
\hline ASSESS & Analytical System and Software for Evaluating Safeguards and Security \\
\hline ATLAS & Adversary Time-Line Analysis Software \\
\hline BHEP & baseline human error probability \\
\hline CDP & critical detection point \\
\hline DEPO & Design and Evaluation Process Outline \\
\hline DOE & U.S. Department of Energy \\
\hline DPA & Diversion Path Analysis \\
\hline EASI & Estimate of Adversary Sequence Interruption \\
\hline $\mathrm{ECP}$ & entry control point \\
\hline ESD & event sequence diagram \\
\hline HEP & human error probability \\
\hline HRA & human reliability analysis \\
\hline LHS & Latin Hypercube Sampling \\
\hline MAA & material access area \\
\hline MAI & material assurance indicator \\
\hline $\mathrm{MC}$ & material custodian \\
\hline MC\&A & material control and accounting \\
\hline $\mathrm{MCM}$ & material control manager \\
\hline MSET & MC\&A System Effectiveness Tool \\
\hline NMF & nuclear manufacturing facility \\
\hline NNSA & National Nuclear Security Agency \\
\hline NPP & nuclear power plant \\
\hline NRC & U.S. Nuclear Regulatory Commission \\
\hline PA & protected area \\
\hline PPS & physical protection system \\
\hline PRA & probabilistic risk assessment \\
\hline RFT & response force time \\
\hline S\&S & safeguards \& security \\
\hline
\end{tabular}




\section{Chapter 1: Introduction}

Safeguards and security (S\&S) systems for nuclear facilities are required to protect nuclear materials from theft, sabotage, and other malevolent human acts. Generally, a site's S\&S system is comprised of four overlapping components: physical protection, material control and accounting (MC\&A), personnel security and information security. The physical protection system (PPS) for a facility is evaluated using probabilistic analysis of adversary paths on the basis of detection, delay, and response timelines to determine timely detection. The path analysis methodology focuses on a systematic, quantitative evaluation of the physical protection component of the system for potential external threats, and often calculates the probability that the PPS is effective in defeating an adversary who uses that attack path (probability of effectiveness, $P_{E}$ ). This effectiveness measure is the degree to which the PPS can protect a broad spectrum of targets against a wide range of potential threats. Other qualitative approaches have been used for MC\&A, personnel security, and information security components of the S\&S protection system [1-4].

Insider adversaries represent formidable threats to the protection of critical assets, including information and materials. This threat takes many forms ranging from petty theft and fraud to theft of critical assets to espionage and terrorism. Depending on their positions, insiders can be very capable security threats because they have knowledge of

operations and the opportunity to access target materials. For facilities that have security systems in place to protect critical assets, these individuals have access "inside" the protective measures. They can take advantage of opportunities that arise to circumvent system elements or to exploit system vulnerabilities and access a target directly without being detected. The detection and delay timelines are not as relevant because insiders 
can choose the most opportune times and optimum strategies, often using protracted or discontinuous attacks. One strategy for addressing the insider threat would be to optimize the control and accountability of materials, and to more fully account for MC\&A elements in the evaluation of the effectiveness of the S\&S protection system.

\subsection{Material Control AND AcCounting Protection System}

S\&S requirements for MC\&A primarily address control and accountability functions including access control, surveillance, material transfers, measurements, and physical inventories. MC\&A operations that track and account for critical assets at nuclear facilities provide a key protection approach for defeating insider adversaries. MC\&A functions such as personnel access control and automated surveillance overlap with PPS functions and are addressed by current path analysis methods. Some MC\&A protections are already incorporated, although perhaps not explicitly identified as such, in the current approach to evaluating the effectiveness of a PPS. For example, procedures and authorizations for material transfers are addressed within PPS elements that provide access between protection layers, such as a personnel or vehicle portal. Other operational activities, such as measurements and physical inventories, have been difficult to characterize in ways that are compatible with the path analysis methods that are currently used to systematically evaluate the effectiveness of a site's protection system. "At the very least, the effectiveness of certain elements has not been rigorously quantified; worse, those elements are sometimes ignored, or simply assumed to be effective" [5]. One approach for addressing this gap uses deterministic Material Assurance Indicators (MAIs) as a metric to evaluate MC\&A activities that are involved in protecting nuclear materials $[6,7]$. Initial testing successfully demonstrated that the MAI algorithm is useful for evaluating characteristics of MC\&A system capability, but it is not truly probabilistic. Thus, the MAI algorithm is not compatible with probabilistic path analysis methods. 
Early in the development of the MAI algorithm, it became apparent that MC\&A activities at an item level could be considered a type of sensor system, with both alarm and assessment capabilities that are necessary for detection. The MAI also provides an approach for evaluating an MC\&A system capability to provide detection of an insider attempting theft of nuclear material [7]. In addition, MC\&A activities, from monitoring to inventory measurements, include a variety of methods for providing information about the attributes and location of target materials and for defining security elements useful against insider threats. These activities can also serve to discourage insiders from engaging in malevolent activity and provide many, often recurring opportunities to determine the status of critical items.

Given this characterization of MC\&A activities and the formulation of the existing path analysis methodology, it is reasonable to investigate probabilistic risk assessment (PRA) methods that may be applicable to the development of a probabilistic approach to characterize MC\&A activities and to evaluate the MC\&A component to provide an overall effectiveness measure of the $S \& S$ protection system to address threats from both insider and outsider adversaries.

\subsection{GOAL AND OBJECTIVES}

The goal of this research is to develop a probabilistic basis and a new method to incorporate MC\&A protection elements explicitly within the existing probabilistic path analysis methodology that is used to systematically evaluate the effectiveness of a site's protection system. To accomplish this, three problem areas need to be addressed:

- "Detection" capabilities of MC\&A protections and quantitative probabilities of detection - individually, in combination, and as a function of time;

- Competing delay and detection timelines for insider theft versus facility detection; and 
- Scenario development to integrate the evaluation of PPS and MC\&A protections within physical protection layers.

The objectives of this work include applying PRA techniques to develop approaches to address these problems. Human reliability analysis (HRA) techniques are investigated for characterizing and providing quantitative measures for MC\&A elements in a manner compatible with probabilistic analyses. An object-based state machine paradigm is developed to characterize insider theft as a race against detection by facility MC\&A activities. This paradigm is coupled with nuclear power plant PRA techniques to characterize detection and delay timelines for MC\&A protection elements and provides the framework for applying convolution mathematics to calculate timely detection. Event sequence diagrams (ESDs) are applied to develop evaluation scenarios for insider paths through the PPS and also incorporate MC\&A activities as path elements. The objectives also include demonstrating the new method with an analysis for several hypothetical theft scenarios.

The development of such a probabilistic approach will enable security analysts to explicitly evaluate the effectiveness of MC\&A protections against insider threats similar to the evaluation of outsider threats performed under the existing probabilistic path analysis methodology. Along with the $P_{E}$ for the PPS, the overall result is an integrated effectiveness measure of a protection system that addresses threats from both outsider and insider adversaries.

\subsection{DISSERTATION ORGANIZATION}

This section provides an introduction, overview of an MC\&A protection system, and the goals and objectives of this research. The remainder of this dissertation is organized as follows: 
- Chapter 2 presents background material on physical protection, MC\&A, the path analysis methodology, characterization and evaluation of the insider threat, and risk analysis tools.

- Chapter 3 presents the details of the extended methodology for one MC\&A daily activity in one physical protection layer.

- Chapter 4 presents the analyses used to demonstrate this new methodology for multiple physical protection layers.

- Chapter 5 presents conclusions and recommendations for additional work. 


\section{Chapter 2: Background}

The background for this research covers several topics, including:

- $\quad$ S\&S system assessment methodology,

- Physical protection,

- MC\&A,

- Design and evaluation of a PPS,

- Insider studies, and

- Risk assessment tools.

Each of these topics is discussed in the following sections.

\subsection{SAFEGUARDS AND SECURITY SYSTEM ASSESSMENT METHODOLOGY}

Design and evaluation of S\&S protection systems generally follows a tailored systems engineering process. The system assessment methodology, shown in Figure 1, has evolved as a framework for assessing S\&S systems to protect nuclear assets within the U.S. Department of Energy (DOE) over 30 years [8]. This framework has been adopted in some form by many organizations both in the U.S. and around the world for nuclear and other critical infrastructure facilities [9-15].

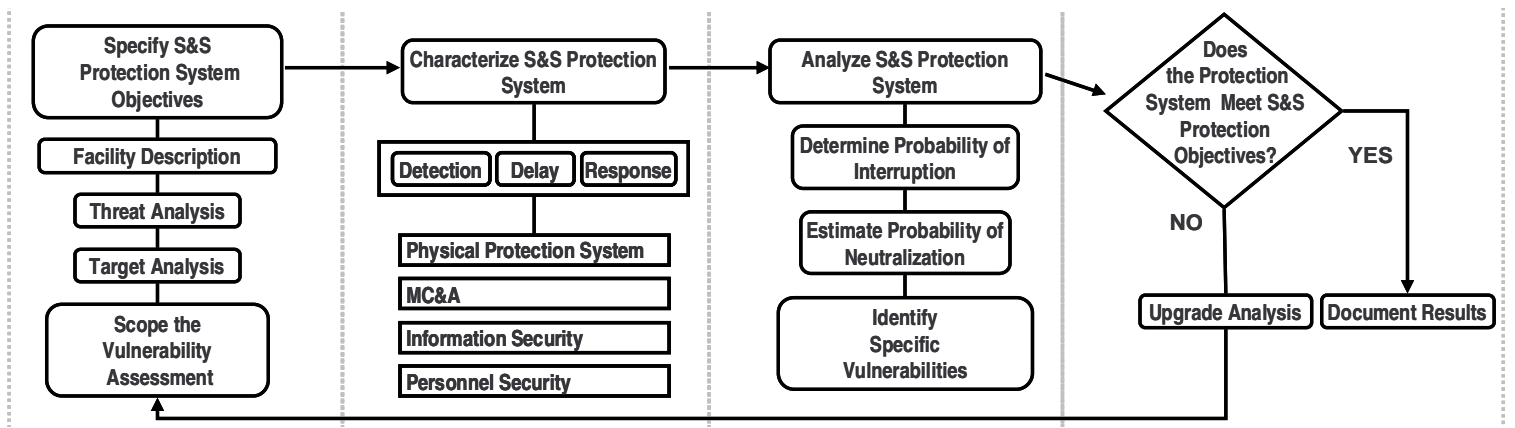

Figure 1. The system assessment methodology used by the U.S. DOE for the design and evaluation of S\&S protection systems [8]. 
Generally, a site's S\&S system is comprised of four overlapping components: physical protection, MC\&A, personnel security and information security. The three integrated functions of all S\&S protection systems, including the physical protection and MC\&A components, are detection, delay, and response. Detection is determining that an unauthorized action has occurred or is occurring. Detection includes sensing the action, generating an alarm signal, communicating the alarm, and assessing that unauthorized actions has occurred. Assessment is when a person determines the cause of an alarm and judges it to be valid or invalid (a false or nuisance alarm). Delay is the slowing down of the adversary's progress toward the objective (theft or sabotage). Characterization of delay establishes the time required by the adversary to bypass or defeat each delay protection element. Common physical delay elements include fences, wall, doors, locks, safes, and active and passive barriers. In combination, delay and detection elements provide layers of protection that extend from a target to the exterior of the site. Response primarily consists of the actions taken by the protective force to prevent adversary success. In this physical response situation, it is important to characterize the response force time (RFT), which is the time elapsed from detection to the response team arriving at the adversary's location with sufficient capabilities to interrupt the adversary's tasks and ultimately neutralize the attack.

\subsubsection{Physical Protection}

For the U.S. DOE S\&S program, physical protection is defined as:

"PHYSICAL PROTECTION. The application of physical or technical methods designed to protect personnel; prevent or detect unauthorized access to facilities, material, and documents; protect against espionage, sabotage, damage, and theft; respond to any such acts should they occur." [16] 
Garcia $[17,18]$ discusses the design and evaluation of a PPS in detail. The purpose of a PPS is to protect important assets from theft, sabotage, or other malevolent attacks. The design of a PPS considers how to combine physical delay elements with sensors, procedures, communication devices, and security personnel to best achieve the overall detection, delay and response functions to meet a protection objective. Evaluation of a PPS design or an existing PPS includes characterizing physical protection elements and their detection, delay, and response functions and determining the PPS effectiveness, usually through a probabilistic path analysis.

\subsubsection{Nuclear Material Control and Accountability}

For the U.S. DOE S\&S program, MC\&A, nuclear materials accountability, and nuclear materials control are defined as follows:

MATERIAL CONTROL AND ACCOUNTING (MC\&A). "Those parts of the safeguards program designed to provide information on, control of, and assurance of the presence of nuclear materials, including those systems necessary to establish and track nuclear material inventories, control access to and detect loss or diversion of nuclear material, and ensure the integrity of those systems and measures." [16]

"NUCLEAR MATERIALS ACCOUNTABILITY. The part of the Material Control and Accountability program encompassing the procedures and systems to:

1. perform nuclear material measurements,

2. verify the locations and quantities of nuclear material through physical inventories,

3. maintain records and provide reports, 
4. perform data analyses to account for nuclear materials and to detect losses, and

5. investigate and resolve apparent losses of nuclear material." [16]

"NUCLEAR MATERIALS CONTROL. The part of the safeguards program encompassing management and process controls to:

1. assign and exercise responsibility for nuclear materials;

2. maintain vigilance over the materials;

3. govern movement, location, and use of the materials;

4. monitor inventory and process status;

5. detect unauthorized activities for all nuclear materials; and

6. help to investigate and resolve apparent losses of nuclear materials." [16]

Over the years, various technologies and methods have been developed and applied to enhance nuclear material control [19-31]. These technologies range from software and systems for continuous monitoring and inventory verification to personnel tracking to monitoring weight and radiation attributes to real-time process monitoring. These technologies are evaluated through testing and demonstration exercises that do not generally address the overall system effectiveness of the MC\&A component of an S\&S protection system. MC\&A procedures and technologies, from monitoring to inventory measurements, include a variety of methods that provide information about the attributes and location of target materials.

More recently, the U.S. DOE's National Nuclear Security Agency (NNSA) has been working to implement the Safeguards First Principles Initiative (SFPI) as a principle-based, risk-management standard for MC\&A programs [20-22]. The SFPI focuses on the effectiveness of the plan and procedures that are developed to implement the requirements of an MC\&A program at an individual NNSA site. The Comprehensive 
Analysis of Safeguards Strategies (COMPASS) model is an MC\&A system effectiveness evaluation tool that has been developed as part of the SFPI initiative to compile site input about nine MC\&A program elements and their respective sub-elements and their ratings for effectiveness weighted by a contribution factor, then provides an overall weighted average that reflects the overall health of the MC\&A program [20]. The COMPASS effectiveness ratings are based on performance data and assessments of the MC\&A program elements and are reviewed by an evaluation board. The effectiveness ratings of the 10-point scale are determined by objective criteria and reflect qualitative ratings of high (8-10), medium (5-7) and low (1-4). The contribution factors are applied as an indication of the relative importance of an element and are determined from a 0-4 point scale, where a factor of 4 is assigned to an element that "provides loss detection or accounts for material" [22]. The SFPI efforts focus on evaluation of the overall programmatic effectiveness of an MC\&A program, the requirements of which include the detection and deterrence of theft and diversion of material [21]. While the SFPI evaluation addresses detection of theft as part of the programmatic requirements, the effectiveness ratings do not reflect the determination of a probability of detection that material is missing or do not specifically address insider theft scenarios or integration with PPS elements.

\subsection{Design ANd Evaluation OF A Physical Protection System}

Garcia [17, 18] provides a comprehensive discussion of methods and their application for designing a PPS and evaluating its effectiveness. Figure 1 illustrates the overall systems engineering process for an S\&S system. The parallel process flow for the PPS component is the Design and Evaluation Process Outline (DEPO) shown in Figure 2. The effectiveness of a site's protection systems is systematically evaluated using qualitative and/or quantitative techniques and is often calculated as the probability of PPS 
effectiveness $\left(P_{E}\right)$, which is a measure of the degree to which the system can protect a broad spectrum of targets against a wide range of potential threats. The DEPO methodology focuses on a systematic quantitative evaluation of the physical protection component of the S\&S system for attack by potential outsider adversaries, whereas other qualitative approaches have been used for MC\&A, personnel security and information security protection systems.

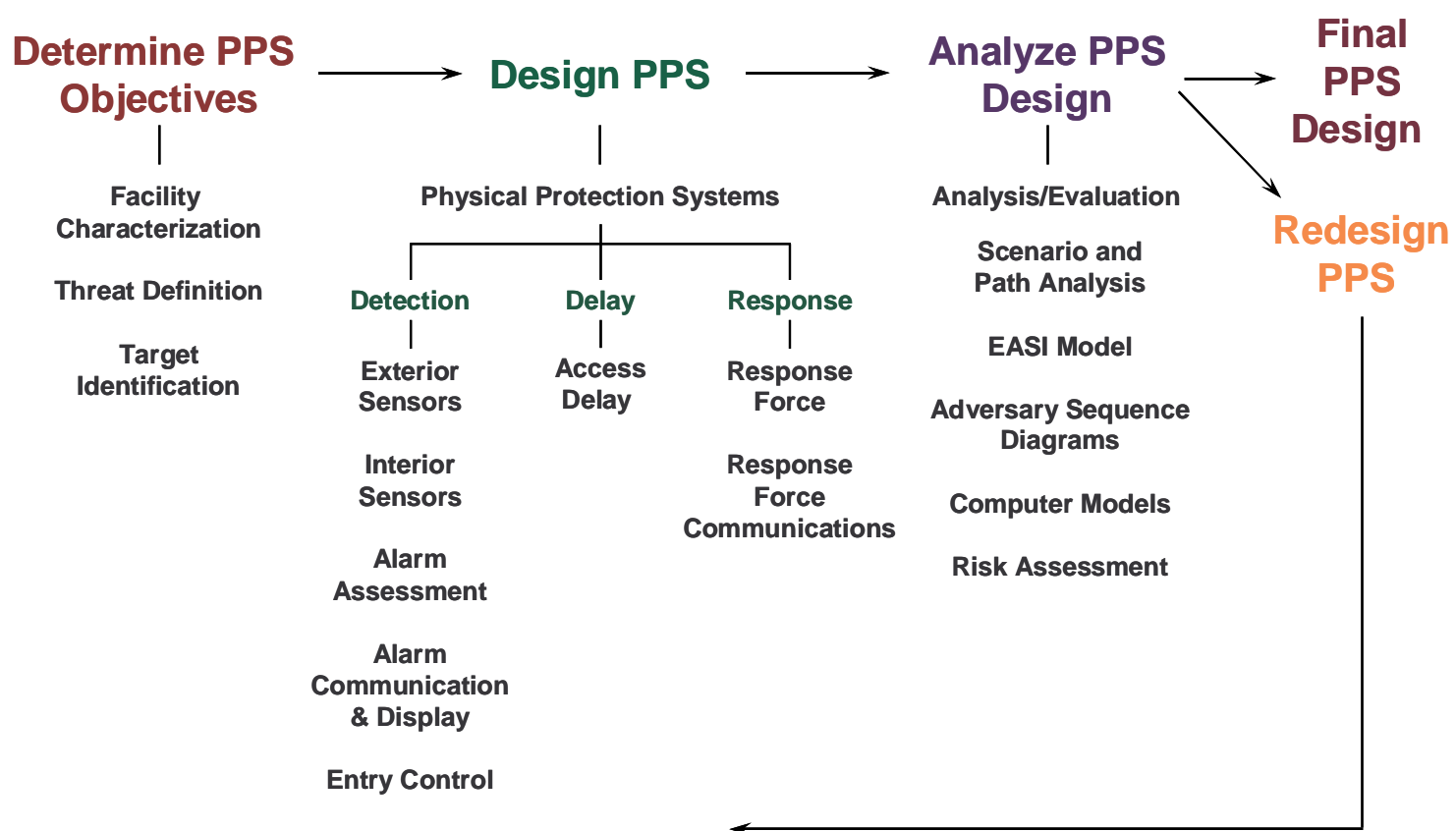

Figure 2. Design and evaluation process outline for physical protection systems [17].

The goal of DEPO is to systematically evaluate the effectiveness of a site's S\&S PPS using objective performance criteria. In this context, an effective PPS consists of protection elements that provide

- timely and accurate detection and assessment of undesired acts,

- timely communication of this information to a response component,

- mechanisms that delay adversaries long enough for the response component to intervene, and 
- a response component capable of preventing adversaries from completing undesired acts.

The overall effectiveness of the system depends upon the performance of each of the components individually, as well as the interaction and performance of the system as a whole. If the site does not meet the protection objectives, a vulnerability assessment [18, 32] identifies specific PPS weaknesses that could potentially be exploited by malevolent threats. PPS upgrades are then implemented to achieve a system effectiveness that meets the protection objectives.

The remainder of this section focuses on the quantitative analysis methods to evaluate system effectiveness for the PPS component. To determine the effectiveness of a PPS, path analysis is performed to evaluate adversary paths and the associated detection, delay and response timelines. The facility is characterized in terms of physical areas, protection layers, protection elements, path elements, path segments, and target locations. Each protection layer contains delay and detection protection elements that define the path elements and path segments of possible adversary paths. Figure 3 illustrates the physical areas of a facility and includes an example of adversary paths. Figure 4 illustrates the physical areas and protection elements as an adversary sequence diagram (ASD). $\quad P_{I}$ is the probability of interruption of the adversary's progress. Path analysis determines $P_{I}$ as a quantitative measure of timely detection on an adversary path. "Timely detection is the principle that system effectiveness is measured by the cumulative probability of detection at the point where there is still enough time remaining for the response force to interrupt the adversary" [17]. This point in the timeline is defined as the critical detection point (CDP). 


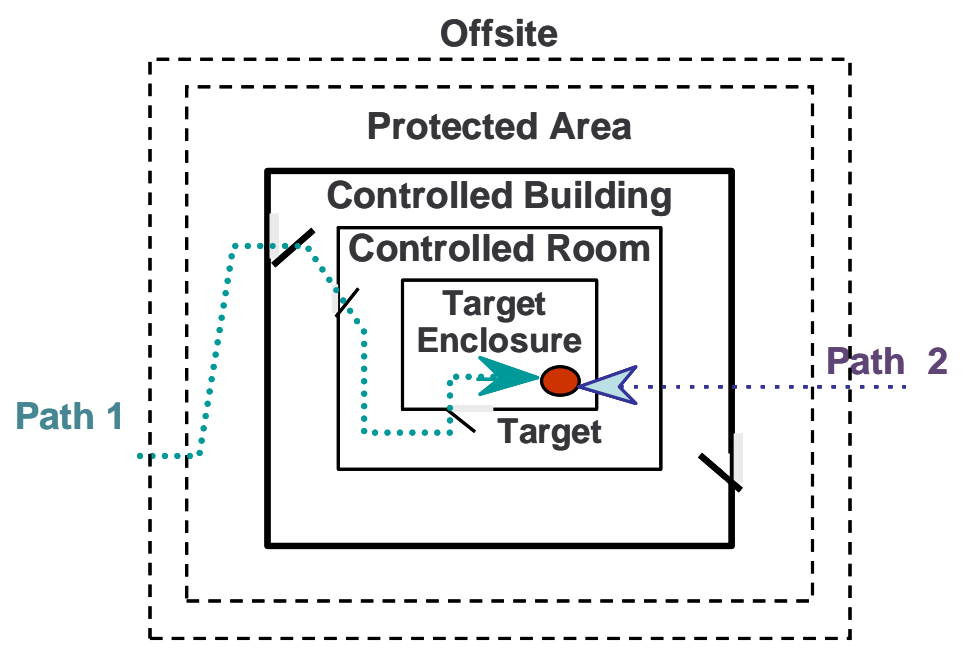

Figure 3. Representation of an example facility's physical areas and possible adversary paths to a target [17].

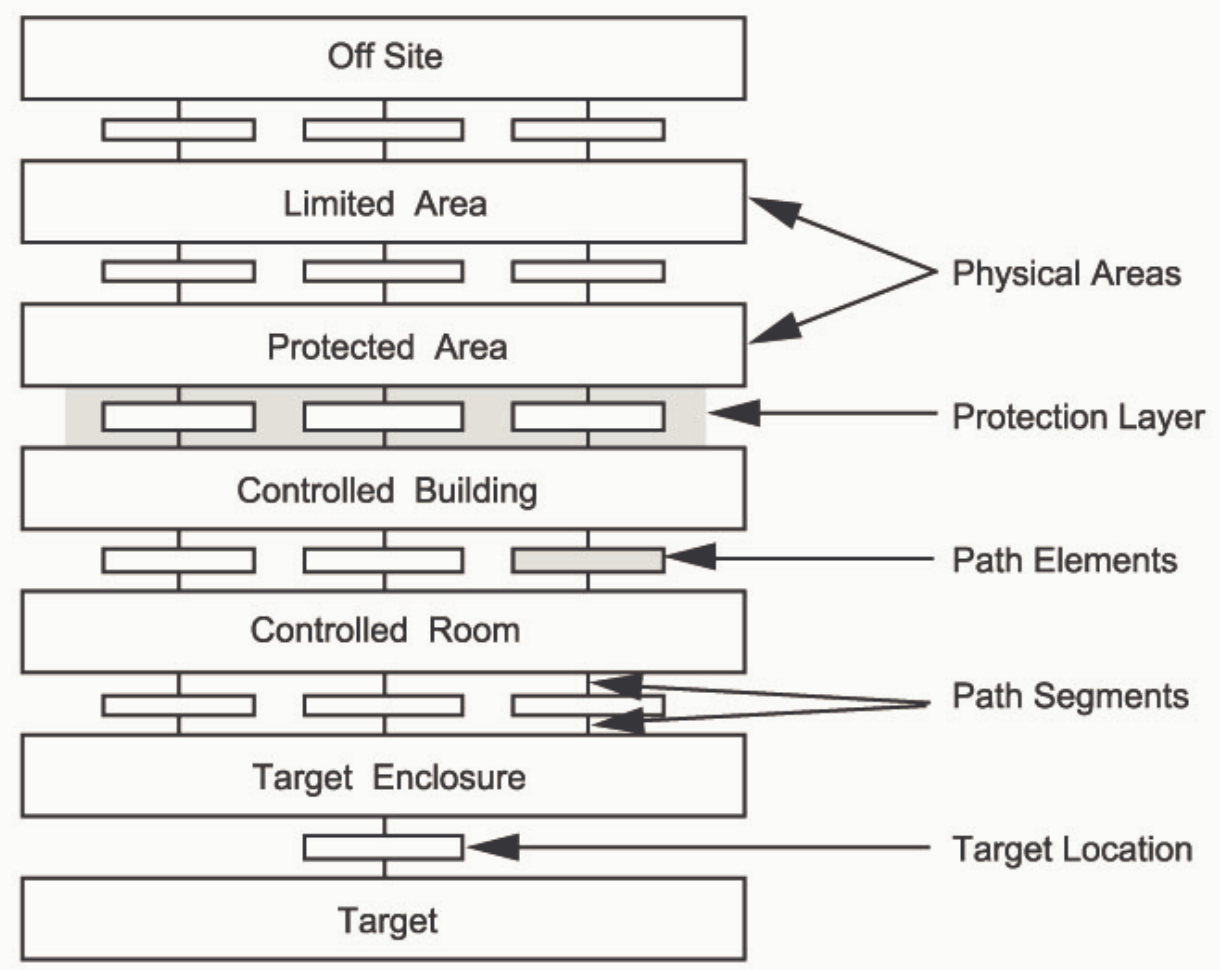

Figure 4. Basic adversary sequence diagram for a facility [17]. 
For each adversary path, path element data are used to calculate a delay time, $T_{R}$, (calculated as a sum) and a probability of detection, $P_{D}$, (calculated as a product,):

$$
\begin{aligned}
& T_{R}=\sum_{i=k}^{m} T_{i}>T_{G} \\
& P_{D}=1-\prod_{i=1}^{k} P_{N D_{i}}
\end{aligned}
$$

where:

$m \quad=$ the total number of protection system elements along the path

$k=$ the point at which the delay time, $T_{R}$, just exceeds the response force time, $T_{G}$

$T_{i}=$ the minimum time delay provided by element $\mathrm{i}$

$P_{N D i}=$ the nondetection probability provided by element $\mathrm{i}$ (that is, the probability that element i will not detect the defined adversary), which is the complement of $P_{D}$

For example, a nondetection probability of 0.2 means that there is a $20 \%$ probability the adversary will not be detected; hence there is an $80 \%$ probability that the adversary will be detected. Note that the analysis models use the probability of nondetection, while $P_{D}$ is the performance measure for detection elements. Detection at each element is assumed to be an independent variable. $P_{I}$, the probability of interruption, is the cumulative probability of detection for all elements up to the CDP.

Depending on the target(s) of interest, protection elements, adversary objectives, and response tactics, among other things, many adversary paths can be defined for a given facility. The critical path for a system is the path with the lowest $P_{I}$. The overall system effectiveness, then, is determined by the $P_{I}$ for this critical path:

$$
P_{E}=P_{I} \times P_{N}
$$

where $P_{N}$ is an estimated probability of neutralization, a measure of the response to the attack. Figure 5 illustrates an example adversary event timeline. In this example, the 
adversary must penetrate two protection layers, the portal and the vault wall, to reach the target material.

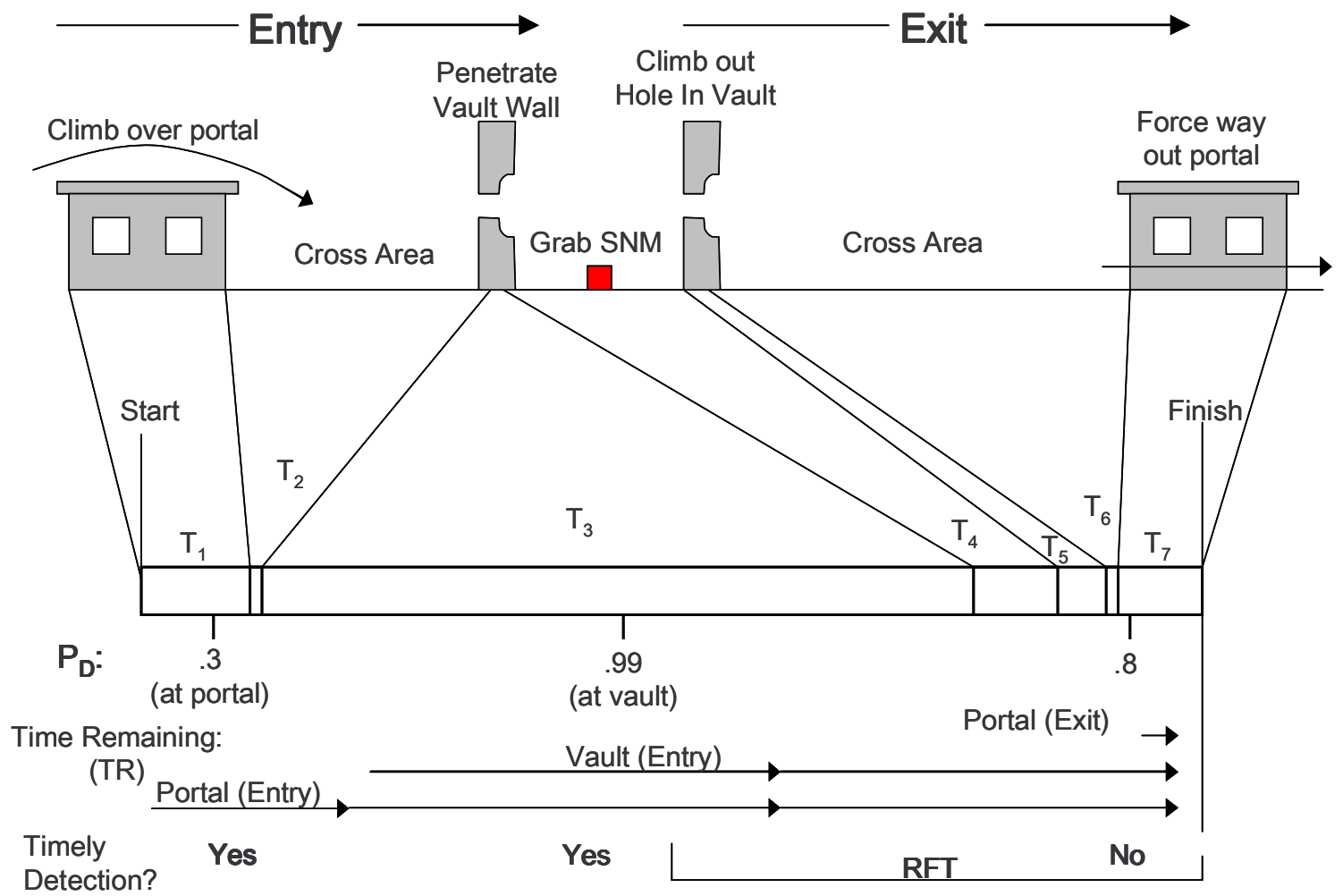

Figure 5. Example adversary event timeline [32].

The actual path analysis for a facility can prove to be complex given the range of targets, objectives, protection elements, and path combinations that must be considered. Several tools have been developed to automate the path analysis. The EASI (Estimate of Adversary Sequence Interruption) approach to physical security evaluation [33] was developed to be executed on a hand-held calculator. Currently, a Microsoft ${ }^{\circledR}$ Excel spreadsheet template is available to implement EASI [17]. SAVI (Systematic Analysis of Vulnerability to Intrusion) is another modeling code that provides a comprehensive analysis of adversary paths into a facility [34]. The ASSESS (Analytical System and Software for Evaluating Safeguards and Security) software includes modules and a 
baseline performance database to characterize the PPS elements of a facility as well as perform the path analysis calculations [35]. The ATLAS (Adversary Time-Line Analysis System) software [36] uses the same models as ASSESS, extends some of those capabilities in the Facility and Outsider assessment modules, and provides updated graphics, computational algorithms, and documentation capabilities. ATLAS, however, does not yet include a complete capability for insider analysis.

The risk equations associated with the calculation of system effectiveness are defined as follows [17]. First, the risk is defined in terms of the probability of an attack occurring $\left(P_{A}\right)$, the probability of success of the attack $\left(P_{S}\right)$, and the consequences $(C)$ of the attack:

$$
R=P_{A} \times P_{S} \times C
$$

Because of the difficulties and uncertainties in determining probabilities of adversary attacks, the conditional risk $\left(R_{C}\right)$ was adopted, that is, $R_{C}$ is conditional on an attack occurring. In addition, using the complement of the probability of an adversary attack in terms of the system effectiveness gives:

$$
R_{C}=\left(1-P_{E}\right) \times C
$$

Once the system effectiveness has been determined, the overall conditional risk can be determined incorporating consequences of the adversary attack for the critical path.

\subsection{INSIDER STUDIES AND EVALUATION OF INSIDER THREATS}

Insiders are the most capable of security threats to any organization. An insider is defined as anyone with knowledge of, access to, and authority at a facility [17]. This definition implies that every employee in an organization is an insider, and any employee may pose an insider threat. For facilities that have security systems in place to protect critical assets, insiders have access "inside" the protective measures. In addition, 
contractors, suppliers, vendors, visitors, and others who are not direct employees of an organization may also be considered a part of the population that has access inside an organization and pose an insider threat. Of concern is a malicious insider who might attempt theft of critical assets, sabotage of equipment or operations, or other criminal activities. The insider threat is a critical concern because successful attacks at secure facilities almost always require the participation of a willing insider.

For theft or diversion of material, malicious insiders are formidable threats because they have knowledge of operations and access to critical areas where target materials may be located. They can take advantage of abnormal conditions (e.g., alarms) or opportunities that arise to circumvent system elements and to access a target directly without being detected. Detection and delay timelines are not as relevant because insiders can choose the most opportune times and optimum strategies, often using protracted or discontinuous attacks. One strategy for addressing the insider threat would be to optimize the control and accountability of materials, and to more fully incorporate MC\&A elements into the evaluation of the S\&S protection system.

Analysis of and protection against insider threats [37-41] can be challenging because insiders have knowledge of operations and opportunity to access critical areas. They can exploit this knowledge, opportunity and access to plan and implement an attack. They are willing to abuse their access to handle material or monitor alarms. Insider studies demonstrate that property theft is prevalent, and a majority of incidents involve a single insider or insiders in collusion, in many cases with outsiders [37-39].

Malevolent insiders may be internally motivated or externally coerced [32]. Figure 6 illustrates characterization of malevolent insiders. Categories of malevolent insiders include "passive" individuals who are willing only to provide information or "active" individuals who will facilitate access or bypass or disable equipment. Active, 
non-violent individuals actively participate in the attack, but are unwilling to use force, while active, violent individuals are willing to use force to achieve their goals. All malevolent insiders use stealth and deceit and do not want to have their activities detected. They may also be rational or irrational; an irrational insider may not seem to use clear decision rules.

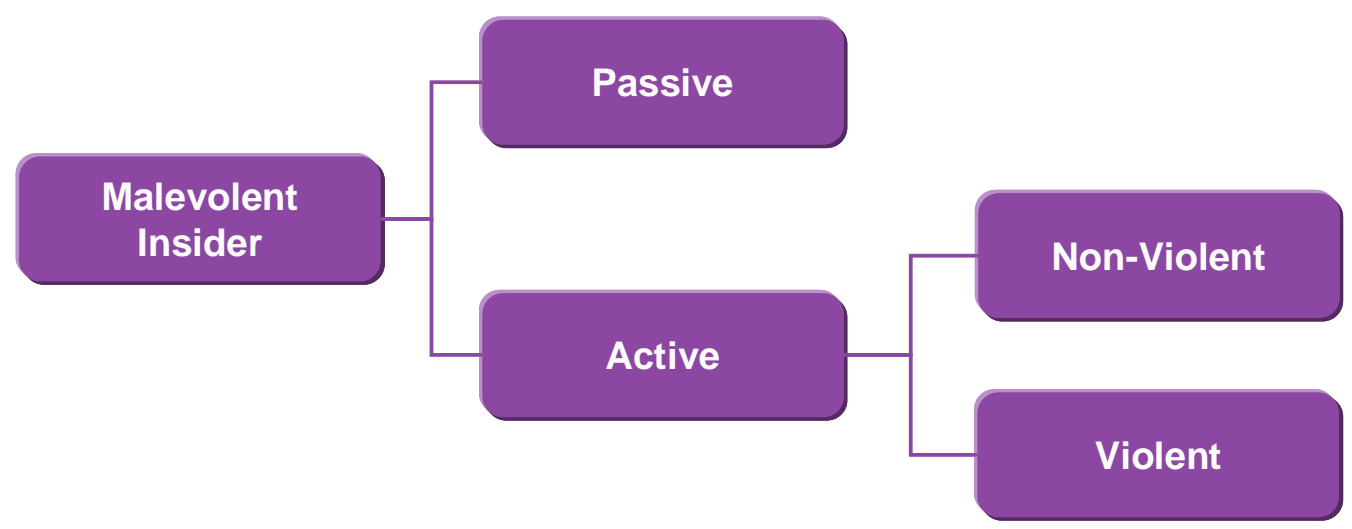

Figure 6. Categories of malevolent insiders.

\subsubsection{Insider Analysis with the Current Path Analysis Methodology}

The path analysis described in Section 2.2 for an outsider threat can also be used for the active, violent insider threat. Variations of this analysis, quantitative and qualitative, are used for various other types of insider threats. For insider attacks, detection and delay timelines are not as relevant because insiders can choose the most opportune times and optimum strategies, often using protracted and discontinuous attacks. In the case of Equation 2 above, determining the probability of detection can be difficult for insider attack scenarios. In many cases, qualitative information about the level of access, knowledge, detection likelihoods, and the resulting effectiveness are rated as low, medium, or high. In other cases, subject matter experts can be used to estimate quantitative detection probabilities. 
Generally, for an insider, $P_{I}$ is the probability of detection, so, from Equation 3:

$$
P_{E}=P_{D} \times P_{N}
$$

where:

$P_{D}=$ conditional probability of detection given that both sensing and assessing the adversary have occurred

$P_{N}=$ conditional probability of neutralization by the response force given that the attack has been interrupted

In the case of the passive or active nonviolent insider, the adversary does not put up a fight, so the threat is neutralized as soon as detection occurs $-P_{N}$ is certain, that is equal to 1 , so,

$$
P_{E}=P_{D}
$$

While the insider analysis method does provide an analysis of the insider threat within the framework for evaluating the effectiveness of the PPS, it does not specifically address the effectiveness of the MC\&A component of an S\&S protection system.

\subsubsection{Other Insider Assessment Methods}

In the late 1970s, the U.S. DOE developed and used the Diversion Path Analysis (DPA) methodology [42] specifically to evaluate the capability of the MC\&A subsystem to detect the diversion of nuclear material by a knowledgeable insider. The methodology used an iterative process to analyze general diversion paths for each material in each process area of a facility to derive a relative path weight based on attributes of the diversion path. The relative path weight is a measure of the complexity of the path rather than a measure of the probability that the insider will chose that path. Of concern was theft of amounts of material attractive for making a crude nuclear explosive device. 
Theft of other types and quantities of nuclear material and performance of the PPS were not addressed by the DPA.

The Insider Safeguards Effectiveness Model is another model developed in the late 1970s [43] to evaluate the effectiveness of a facility's safeguards against a group of insiders attempting theft or sabotage. The model requires user input, which in most cases is very subjective. Safeguards Evaluation Tool (ET) [44] was another methodology and computer tool that was developed as part of the subsequent Safeguards Evaluation Method for nonviolent insider adversaries. The path analysis tools described in Section 2.2 (EASI, SAVI, and ASSESS) have also been applied to insider analyses, specifically for a non-violent insider adversary on an exit path from the facility.

\subsubsection{Material Assurance Indicator Algorithm Development}

Prior to the work of Dawson and Hester [6,7], no measures or standards for comparison were defined to determine whether a protection system provided effective control of nuclear materials, that is, the effectiveness of an MC\&A system. The development of the MAI for evaluating the MC\&A activities involved in protecting nuclear materials has shown promise for providing this type of metric [6, 7]. A perfect materials control system would ensure that all the attributes and each location of materials in a system are known all the time. In the case of evaluating the MC\&A

component of an S\&S system, the materials information would be evaluated within the timeline for an adversary attack. The MAI algorithm computes an MAI on a per-item basis and indicates material assurance at any given time. Items can be defined as the container of a group of items or the physical containment of multiple items, such as a vault configuration. The two-part formulation accounts for the attributes, locations, and time interval of materials: 


$$
\begin{aligned}
M A I & =\frac{\sum_{i}^{N} M C F_{i} \times\left[\left(H_{R}, A_{R}, R_{R}\right) \times L F_{i}\right]}{N} \\
L F_{i} & =\frac{\Delta t}{\max (t, \Delta t)}
\end{aligned}
$$

where:

MAI = Material Assurance Indicator - the metric for assessed detection

$M C F=$ Material Characterization Factor - what is the item to be protected

$H_{R} \quad=$ Handling - where the item is located

$A_{R} \quad=$ Attribute Monitoring - where the item is located

$R_{R} \quad=$ Gamma/Neutron Monitoring - where the item is located

$L F \quad=$ Latency Factor - when the material was last handled or monitored

$\Delta t \quad=$ Critical time - based on protection strategies

$t=$ Time when the last handling/monitoring occurred, subtracted from $\Delta \mathrm{t}$

$N=$ Number of items defined

Values for $M C F$, handling, and monitoring are determined by relative rankings of various MC\&A procedures and technologies, on a scale of $[0,1]$, yielding an overall measure between $[0,1]$. The relative ranking is determined by subject-matter experts and verified through experimental results. An informal elicitation was used to determine an initial set of values for initial algorithm development and testing.

The algorithm was tested for four different scenarios at hypothetical facilities: to use real-time information on an item basis to improve decision making on response methods, to track unauthorized movement of material and heighten alert to increase $\mathrm{P}_{\mathrm{I}}$, to determine the frequency of a physical inventory given the failure probability of sensors in a monitoring system, and to address the performance of MC\&A protections. The initial testing demonstrated that the algorithm shows promising capabilities to provide positive 
responses for each of the four scenarios. Also, early in the development of the MAI algorithm, it became apparent that activities at an item level could be considered a type of sensor system, with both alarm and assessment capabilities that are necessary for detection. The MAI algorithm can also evaluate MC\&A system capability to provide detection of an active non-violent insider attempting theft or diversion of nuclear material.

The algorithm is currently formulated as a deterministic point estimate for an individual item or group of items, separate from the path analysis methods for determining system effectiveness of a PPS. A probabilistic analogue for the MAI will enable security analysts to explicitly incorporate MC\&A protections into the $P_{E}$ calculations performed for the existing probabilistic path analysis methodology to provide an effectiveness measure of both the physical protection and MC\&A systems to address outsider and insider threats.

\subsection{RISK ASSESSMENT TOOLS}

Given the techniques used in the probabilistic path analysis methodology, it is reasonable to investigate other applications of PRA that may be applicable to the development of a probabilistic analogue for the MAI. Since the WASH-1400 study [45], PRA methods have been developed for and applied to for the assessment of nuclear power plant safety. A summary of these methods for the subsequent severe accident risk study (NUREG-1150) is provided in Breeding, et al. [46]; the South Texas Project nuclear power plant also describes the details of PRA methods [47]. In the early 1990s, the U.S. Nuclear Regulatory Commission (NRC) developed and adopted in 1995 a policy statement regarding the expanded use of PRA and associated analyses [48] that has led to a wider implementation of risk-informed decision-making. PRA techniques have also 
been widely applied in the chemical processing, aerospace, aviation, and maritime safety industries [49-52].

More recent work has applied PRA approaches to the evaluation of proliferation resistance evaluation [53-56]. These efforts have employed a Markov modeling approach for proliferation resistance in advanced fuel cycles consistent with the evaluation framework being developed by the Proliferation Resistance and Physical Protection Expert Group of the Generation IV International Forum [57]. The initial efforts [53, 54] investigated the application of a Markov chain method to perform detailed proliferation scenario and pathway analysis and to quantify measures of proliferation resistance, including proliferation success, probability of detecting proliferation, technical difficulty, and proliferation time. Analyses have been performed for misuse, diversion from the front-end and back end of the fuel cycle, and abrogation scenarios for an advanced lightwater reactor [53, 54], different reprocessing facilities [53], and an example sodium fast reactor $[53,55]$. The Markov chain method has the capability to account for some of the dynamic features of proliferation, including the large number of uncertainties, the unpredictability of human performance, and the effect of changing conditions with time $[54,56]$. More recently, safeguards approaches, false alarms, concealment, and human performance have been incorporated in the Markov modeling [54], and four different fuel cycle arrangements have been analyzed to determine proliferation success and proliferation risk, where consequence is represented by a material type index [56]. The proliferation resistance problem has many similar characteristics to insider theft. The Markov models described in these papers, however, are continuous-time models that are solved as a system of continuous differential equations in time. With this solution approach, hard delays that are characteristic of discontinuous insider theft scenarios would be difficult to model. In addition, the Markov modeling approach is less 
compatible than other approaches to the existing path analysis methods used to evaluation system effectiveness of a PPS.

Other recent work has applied PRA techniques to develop a fault tree for a functional MC\&A model, including basic event probabilities determined by a Delphi expert judgment process to evaluate MC\&A effectiveness and relative risk calculations performed using PRA software [58-62]. The functional model for the MC\&A System Effectiveness Tool (MSET) details 144 fundamental elements of a comprehensive MC\&A system, including key functions to deter, detect, and mitigate potential insider threats $[59,60]$. Quantitative values for the basic event probabilities are converted from qualitative responses to a survey questionnaire about MC\&A elements at a facility [60] using a Delphi process to combine values provided by multiple experts. The fault tree, based on the functional model, along with basic event probabilities indicative of "operational quality" derived by experts are used to assess the basic reliability of the MC\&A system at a nuclear facility [59]. The results of the PRA calculations using the fault tree provide relative risk measures, and an estimate of the overall failure probability "to maintain nuclear material under the purview" of the MC\&A system [59]. Addressing the insider threat using the MSET model has been explored by examining "those elements, which based on expert judgment, are most attractive to and vulnerable to insiders," [59] but determination of detection probabilities, analysis of insider theft scenarios analyses, or integration with PPS elements are not addressed.

Of the many applications of PRA that were investigated, the techniques that were identified to support the probabilistic basis for incorporating MC\&A protections into the existing path analysis methodology include techniques for variable event sequence ordering and HRA techniques for determining detection probabilities for MC\&A activities. 


\subsubsection{Techniques for Variable Event Sequence Ordering}

The path analysis performed to evaluate a PPS can be represented by a traditional PRA event tree with binary branching for detection and non-detection through each protection element of an adversary's path. To incorporate MC\&A activities that may be characterized as having recurring "detection" opportunities, techniques for variable event ordering need to be applied. The Object-based Event Sequence Tree methodology [63] combines the best features of traditional event tree analysis and Monte Carlo-based event simulation with concepts from object-oriented analysis into a PRA technique that easily supports recurring or variable event ordering. Developing an object model provides a framework for characterizing insider theft scenarios that include recurring MC\&A activities. The set of possible scenarios to be evaluated can be deduced by analyzing the object model as an event sequence diagram (ESD) that extends the traditional event tree representation of insider theft to include MC\&A activities. ESDs are another PRA technique that are used to represent the variability and uncertainty of events in accident scenarios analyzed for safety analyses of space craft launches [50].

\subsubsection{Human Reliability Analysis Techniques}

Since the early 1970s, HRA has been considered to be an integral part of PRA for a nuclear power plant (NPP). Human performance in NPP operations continues to be an important element for reactor safety. Swain and Guttmann [64] developed a handbook that includes methods, models, and estimated human error probabilities (HEPs) to address human performance of operations for PRA of an NPP. The methods in the handbook describe various approaches for representing human error in a PRA. The frameworks for incorporating HRA in a PRA has evolved from Swain's and Guttmann's Technique for Human Error Rate Prediction [64] that considers how performance shaping factors (stress, workload, training) influence the occurrence and type of human error 
mechanisms to more multi-disciplinary approaches that more fully consider the how human factors, behavioral science and plant engineering contribute to plant conditions that influence not only performance shaping factors, but also specific error mechanisms and unsafe actions ("errors of commission") that contribute to accidents $[65,66]$.

Most applicable to establishing a probabilistic basis for incorporating MC\&A activities with physical protection are Swain's and Guttmann's methods for checking operations as recovery factors. A recovery factor is defined as "an element of an NPP system that acts to prevent deviant conditions from producing unwanted effects" [64, p. 19-1]. Human redundancy is a type of recovery factor that occurs when one person checks his or her own work or another person's work, detects an error that has occurred and corrects it. The handbook describes a variety of checking operations used in an NPP. Some may involve checking routine tasks that recur on a regular basis performed by the same or different persons with or without a written checklist. Others may involve one person checking another person's work; special short-term, one-of-a-kind checking with alert factors; or special measurement tasks. HRA methods for evaluating operator attention to unannuciated alarm signals during nuclear power plant operations also provide insights for addressing MC\&A activities. These methods also show how the effectiveness of repeated inspections decreases over time if an anomalous condition is not recognized the first time it occurs. 


\section{Chapter 3: Methods for Extended Path Analysis - One Daily MC\&A Activity in One Physical Protection Layer}

This work focused on a new method to incorporate MC\&A protection elements within the existing probabilistic path analysis methodology to estimate $P_{E}$ for insider threats. The approaches taken to complete this work included:

- The use of available path analysis modeling techniques

- The characterization of MC\&A activities

- The investigation of safety PRA methods as the basis for possible applicable analogues

- The use of applicable statistical analysis techniques to investigate the development of detection distributions for MC\&A elements

- The development of data sets for representative hypothetical facilities

- The use of available path analysis modeling and computational tools to demonstrate comparative $P_{E}$ calculations

Three important insights resulted from the initial investigation of MC\&A protection elements. These insights and how these might be incorporated in existing path analysis modeling techniques include:

1. MC\&A protection elements are interwoven within each physical protection layer, and provide additional detection and delay opportunities within the S\&S system. In their MAI work, Dawson and Hester $[6,7]$ observed that many MC\&A activities provide sensing and detection capabilities, similar to other sensors in a PPS. In addition, MC\&A activities that discourage insiders provide many, often recurring opportunities to determine the status of critical items (for example, daily administrative checks). 
2. MC\&A protection elements can act as a "switch" that changes the state of the facility from normal operation to one of heightened alert when material is discovered "missing."

3. Insider theft can be characterized as a "race" between insider theft stages that move target material from internal to external physical protection layers and the MC\&A protection elements that detect that material is not where it should be.

These insights along with the identified PRA techniques provided a basis for characterizing MC\&A activities in a way that is compatible with the existing path analysis methodology.

\subsection{OBJECT-BASED PARADIGM FOR INSIDER THEFT}

Considering the insights and observations about MC\&A protection elements as well as the characteristic differences with respect to delay and detection timelines for insider scenarios and the relationship to protection layers, an object-oriented modeling approach [63] was applied to develop an object-based state machine paradigm to characterize insider theft scenarios. An example of such an object-based state machine is shown in Figures 7a and 7b. The "system" is characterized by two objects: an Insider Theft object and a Facility Status object. The figures illustrate the state transition diagrams for each object: the Insider Theft object (7a) and the Facility Status object (7b) and their interrelation. Each box in the diagrams is a possible "state" of the object at a given point in time. The arcs between each state are events that can occur to move the object from one state to another.

The Insider Theft object generally describes the possible steps in a specific insider theft scenario. In this example, the adversary must: 


\section{Insider Theft}

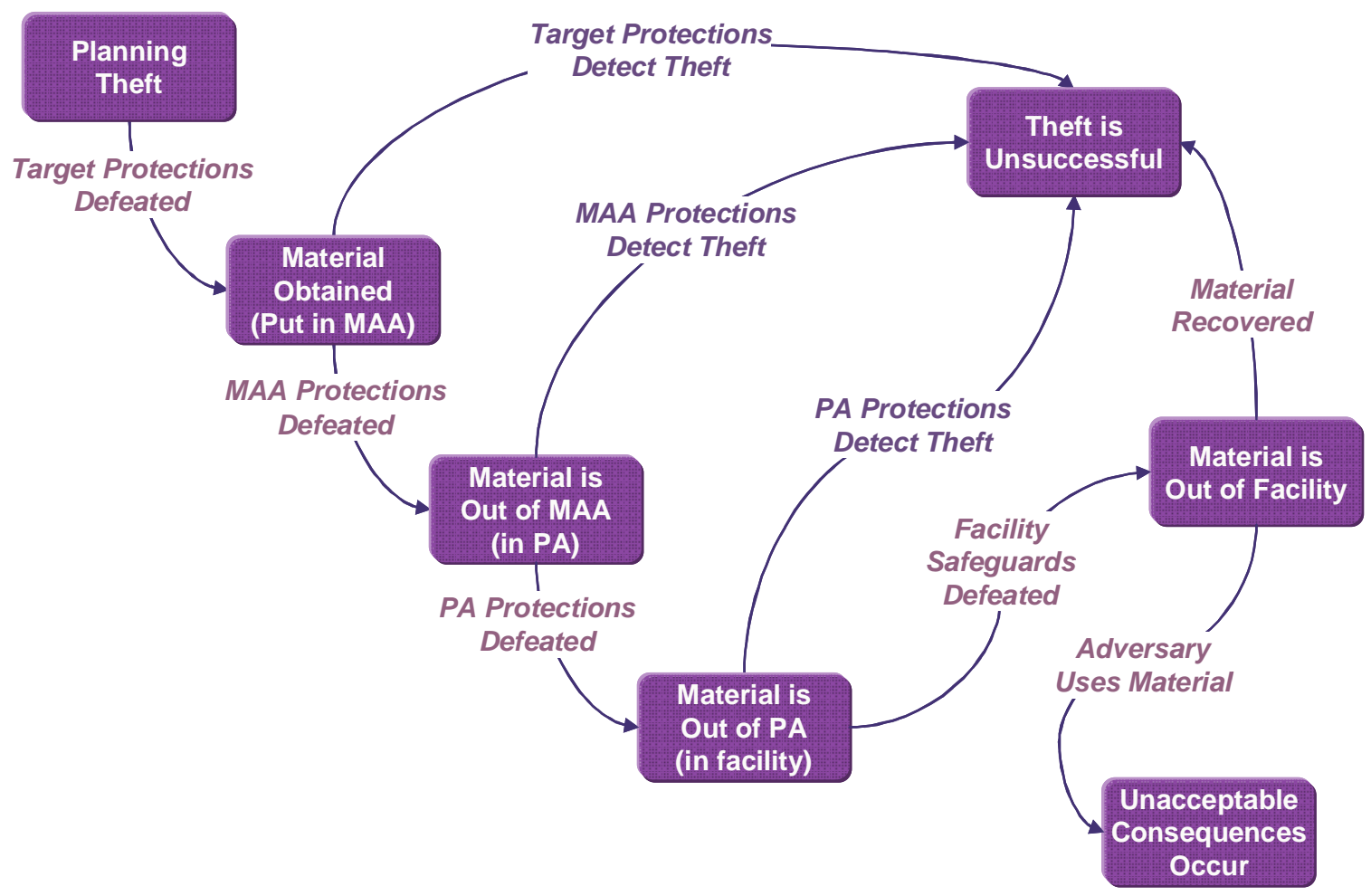

Figure 7a. State transition diagram for Insider Theft Object.

\section{Facility Status}

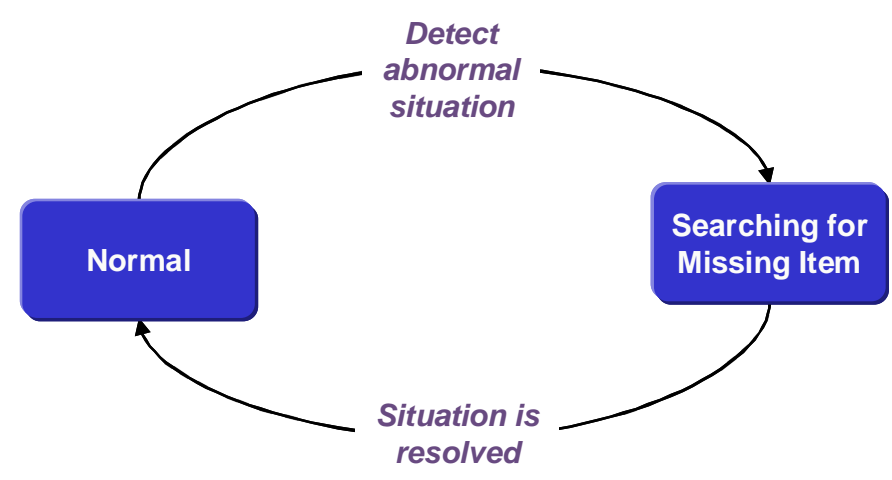

Figure 7b. State transition diagram for Facility Status Object. 
1. defeat safeguards at the target to obtain the material,

2. defeat safeguards in the material access area (MAA), and move the material through the protected area (PA),

3. defeat safeguards in the PA and move material through the facility boundary, and then

4. defeat safeguards at the facility boundary and move the material out of the facility.

The Facility object indicates how MC\&A protection elements act as a "switch" that changes the state of the facility from normal to heightened alert when the facility is searching for material that is discovered "missing."

This model is specifically constructed for each attack scenario, and the defined states and state transitions will vary as appropriate to the modeled scenario. The analytical examples presented in this work end at the state where material is out of the facility, although modeling additional steps in the attack is also possible. This approach characterizes insider theft as a "race" between insider theft stages from internal to external physical protection layers and the MC\&A system elements that detect that the material is not where it should be. This characterization of an insider theft is similar to the characterization of an outsider attack for the PPS as a race between the adversary and facility response team after detection has occurred.

This modeling approach was used to develop an overall understanding of the insider theft and its relationship to the facility state. This state machine could be modeled using discrete event simulation methods that would provide relative probabilities of the final end-states of all possible scenarios. In this work, however, it was important to model, in detail, the intermediate steps of the insider theft scenarios to investigate the 
importance of each MC\&A activity and PPS element in detecting the insider theft actions.

\subsection{INCORPORATING AN ASSESSMENT OF MC\&A ACTIVITIES}

Event trees are often used in evaluating PPS scenarios, but are difficult to use here because traditional event trees do not show dependency among events in a way that is easily summarized by the analyst for a reviewer. Characterizing the protection system to include MC\&A elements interwoven within each physical protection layer provides a basis for extending the traditional event tree representation with detection or no detection of insider theft through the PPS (Figure 8) to include MC\&A activities. The set of possible scenarios to be evaluated can be deduced by analyzing the object model as an ESD that incorporates MC\&A detection with PPS detection. Figure 9 illustrates this extension as an ESD where detection by MC\&A (yellow boxes) and PPS protection elements (white boxes) are considered in each protection layer. The ESD allows a more detailed representation of the steps of insider theft, the incorporation of MC\&A activities within each layer, and event sequence progression for the differing facility state conditions of normal or heightened alert. The ESD also provides a framework for propagating probability values to determine effectiveness for detecting missing material. Figure 9 indicates where MC\&A activities trigger a change of facility state from normal to "heightened alert," when the facility is searching for material that is unaccounted for and may be missing. This state change is modeled using different PPS detection probabilities for the normal and heightened alert facility states at each detection opportunity. Detection probabilities for a "Normal" facility state can be enhanced if an MC\&A alert has occurred and the facility state is "Searching for Missing Material." Logically, if an MC\&A alert has occurred, the facility has a higher probability of 


\begin{tabular}{|c|c|c|c|}
\hline Initiating Event & Layer 1 & Layer 2 & Layer 3 \\
\hline & \multicolumn{3}{|l|}{ Detect (PD1) } \\
\hline & \multirow[b]{2}{*}{ No Detect (1 - PD1) } & \multicolumn{2}{|l|}{ Detect (PD2) } \\
\hline & & \multicolumn{2}{|r|}{ Detect (PD3) } \\
\hline & & No Detect (1 - PD2) & \multirow[b]{2}{*}{ No Detect (1- PD3) } \\
\hline & & & \\
\hline
\end{tabular}

Figure 8. Traditional event tree model of insider theft through protection layers.

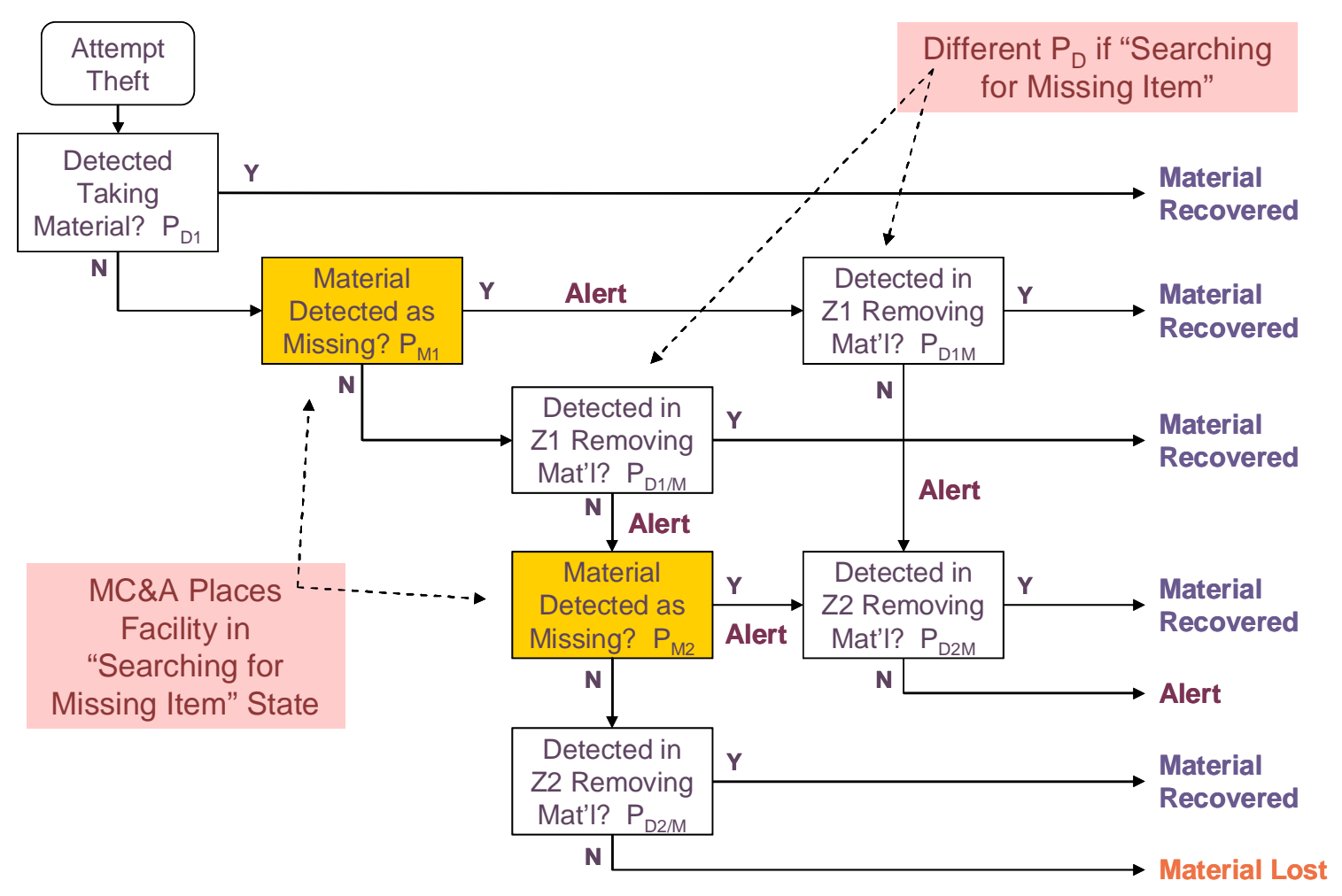

Figure 9. Insider theft modeled as an event sequence diagram (ESD) incorporating MC\&A. 
detecting and finding the material, and the adversary has a lower probability of successfully removing the material from a physical protection layer.

\subsection{INSIDER THEFT AND MC\&A DETECTION TIMELINES}

One of the challenges for evaluating the effectiveness of an S\&S protection system against an insider adversary is that the detection and delay timelines determined for the outside adversary and the PPS are not as relevant because an insider adversary can choose the most opportune time to take advantage of system vulnerabilities. The various theft events may be separated by large gaps in time (discontinuous or protracted theft). The object-based state machine provides a framework for representing the time of occurrence for each step in the theft as well as the MC\&A detection time that changes the facility state as probability distributions. Determining whether theft or detection occurs first, that is who wins the race, is accomplished by convolution of the theft and detection distributions for each scenario.

Time variables are defined for the insider theft timeline and the MC\&A detection timeline. As an insider theft is initiated and proceeds through the physical protection layers of a facility, the insider theft timeline is defined by two (or more) time variables:

$\mathrm{T}_{\mathrm{R} 1}-\quad$ Part of the insider theft timeline that represents the time for the adversary to successfully remove target material from Physical Protection Layer 1. The time interval begins when the adversary obtains the material and ends when the adversary removes target material from Physical Protection Layer 1.

$\mathrm{T}_{\mathrm{Ri}}-\quad$ Part of the insider theft timeline that represents the time for the adversary to successfully remove target material from the $i^{\text {th }}$ Physical Protection Layer. The time interval begins when $\mathrm{T}_{\mathrm{R}(\mathrm{n}-1)}$ ends and ends when the adversary removes the target material from the $\mathrm{i}^{\text {th }}$ Physical Protection Layer for layers 2 through $n$. 
Additional time variables are defined as needed for each stage of an insider theft through additional physical protection layers. Each of these times is represented as a probability distribution in order to represent the variation in both the time before a removal opportunity presents itself and the time to accomplish the removal task. The distributions for the adversary theft timeline $\left[\mathrm{P}\left(\mathrm{T}_{\mathrm{R} 1}\right), \mathrm{P}\left(\mathrm{T}_{\mathrm{Ri}}\right), \ldots, \mathrm{P}\left(\mathrm{T}_{\mathrm{Rn}}\right)\right]$ depend on the defeat methods available to an adversary (e.g., removal through an SNM monitor after disabling the monitor) and when the adversary may take advantage of opportunities to exploit system vulnerabilities or to circumvent protection elements.

The MC\&A detection timeline is defined by the detection opportunities provided by MC\&A activities as they are performed in each physical protection layer and is defined as:

$\mathrm{T}_{\text {MC\&AAlert }}-$ The time when MC\&A activities may indicate that target material is missing. The time interval begins when theft occurs and ends when MC\&A alert occurs.

$\mathrm{T}_{\text {MC\&AAlert }}$ is the time when the Facility state transitions from the "Normal" state to the "Searching for Missing Material" state (Alert). Times and associated probabilities $\left[\mathrm{P}\left(\mathrm{T}_{\mathrm{MC} \& A A l e r t}\right)\right]$ are dependent on specific MC\&A activities included in a scenario. The distribution for the MC\&A detection timeline can be developed considering specific MC\&A activities and associated operational considerations of when and how these activities are performed. In a well-designed MC\&A and security system, $\mathrm{T}_{\text {MC\&AAlert }} \ll<$ $\mathrm{T}_{\mathrm{Rn}}$ to allow for the maximum opportunity to interdict the adversary and stop the theft. If $T_{\text {MC\&AAlert }}>T_{R n}$, then the material has been stolen before the facility is even aware that it is missing. 


\subsection{CONVOLUTION INTEGRAL ${ }^{1}$}

MC\&A activities contribute to the effectiveness of the facility protection system by providing alerts that material may be missing. The effectiveness of MC\&A activities can be determined by comparing the probability distributions for the time for MC\&A alerts $\left[\mathrm{T}_{\mathrm{MC} \& A A l e r t}\right]$ with the probability distributions for the time for removal of material by the adversary $\left[\mathrm{T}_{\mathrm{R} 1}, \mathrm{~T}_{\mathrm{Ri}}, \ldots, \mathrm{T}_{\mathrm{Rn}}\right]$ using probabilistic convolution to determine the probability that detection occurs before theft. As presented in Appendix A, convolution is a method of combining probability distributions that has been used in nuclear power plant PRA [47] and security timeline analyses [33].

As a general example considering removal of material, let $T_{M}$ and $T_{R}$ be random variables over time, where $T_{M}$ is the timing for MC\&A alerts and $T_{R}$ is timing for insider theft (removal of material). Let $t_{M}$ and $t_{R}$ be specific values of these random variables. The range of $T_{M}$ and $T_{R}$ is $[0, \infty)$.

Let $P\left(t_{M}\right)$ denote the probability density function for $T_{M}$ and let $P\left(t_{R}\right)$ denote the probability density function for $T_{R}$. Let $P\left(t_{M}, t_{R}\right)$ denote the joint probability density function for $T_{M}$ and $T_{R}$.

A random variable for time of possible "detection" is defined as $T_{D}=T_{M}-T_{R}$ and $t_{D}$ is a specific value of this random variable. The probability density function for $T_{D}$ is:

$$
P\left(t_{D}\right)=\int_{0}^{\infty}\left\{P\left(t_{M}, t_{R}\right) \mid t_{R}=t_{M}-t_{D}\right\} d t_{M}
$$

If $T_{M}$ and $T_{R}$ are independent, then $P\left(t_{M}, t_{R}\right)=P\left(t_{M}\right) \cdot P\left(t_{R}\right)$, and

$$
P\left(t_{D}\right)=\int_{0}^{\infty} P\left(t_{M}\right) \cdot P\left(t_{M}-t_{D}\right) d t_{M}
$$

The range of $T_{D}$ is $[-\infty, \infty]$. The probability that $T_{D}$ is less than zero is:

1 The formulation for convolution of insider theft and MC\&A detection was developed with the assistance of John Darby of Sandia National Laboratories. 


$$
P\left(t_{D}<0\right)=\int_{0}^{\infty} P\left(t_{D}\right) d t_{D}
$$

This is the probability that an MC\&A alert occurs and the Facility transitions from the "Normal" state to the "Searching for Missing Material" state before the insider is successful in moving the material past that physical protection layer.

\subsection{HuMAN RELIABILITY MODELS FOR MC\&A ACTIVITIES}

The characterization of MC\&A activities as having detection capabilities was a first step for incorporating MC\&A activities as additional sensors in a site's protection system. In addition, a probabilistic basis is needed to determine an appropriate probability of detection $\left(P_{D}\right)$ for MC\&A protection elements. HRA methods of Swain and Guttmann [64], specifically NPP checking operations as recovery factors and the associated HEPs, were applied as a basis to probabilistically characterize MC\&A detection.

\subsubsection{MC\&A Activities as NPP Checking Operations}

MC\&A activities have many similar characteristics to operator tasks performed in an NPP in that the reliability of these activities depends significantly on human performance. Many of the procedures involve human performance in checking for anomalous conditions. As an example, checking the status of a valve in an NPP is similar to checking the status of a nuclear material target in a vault. The respective associated anomalous conditions are that a valve should be closed but is partially or completely open (perhaps after a maintenance activity), and that a target in a vault is not where it should be located. Both can be characterized as checking procedures, in which an identified checking opportunity exists, and a person discovers or fails to discover an anomalous condition. Further characterization of MC\&A activities as procedures that check the 
status of critical assets provides a basis for applying HRA models and methods to determine probabilities of detection for MC\&A protection elements - the probability of detection is defined as the complement of the HEP for performing an operation.

Table 1 identifies typical MC\&A activities and similar characteristics of operator tasks identified by Swain and Guttman [64 Table 19-1]. The table also includes an estimated baseline HEP (BHEP) associated with the NPP operator tasks as determined by the HRA work of Swain and Guttman [64]. These estimated BHEPs can be applied to MC\&A protection elements by using the complement as a probability of detection for a given MC\&A activity.

\subsubsection{Dependence Models for Recurring MC\&A Activities}

Within a PPS, sensor elements are designed to detect unauthorized activity. This work has provided additional insights to characterize MC\&A activities as additional sensors within a site's protection system. MC\&A activities are interwoven within each protection layer of the PPS and provide additional detection and delay opportunities within the S\&S protection system. These activities are important protection elements against insider theft and can serve to discourage malicious insider activity. They provide many, often recurring opportunities to determine the status of critical items (for example, daily administrative checks). As an example, Table 2 lists some key administrative MC\&A activities that are performed on a recurring basis. A year-long detection opportunity timeline can be constructed from the compilation of the recurrence of these activities, which demonstrates the importance of these activities as protection elements against insider threats.

In this work, MC\&A activities have been characterized as a type of human redundancy recovery factor. Generally, MC\&A activities would be considered independent events. However, because many of the MC\&A activities are recurring, it is 
Table 1: $\quad$ Characterization of MC\&A activities as different types of NPP checking operations with estimated probabilities (HEPs) that a checker will fail to detect an error (columns 2 and 3 from [64, Table 19-1])

\begin{tabular}{|c|c|c|}
\hline MC\&A Activity & Nuclear Power Plant Checking Operation & BHEP \\
\hline Plan of the Day & Checking routine tasks using written materials & 0.10 \\
\hline Material Measurement & $\begin{array}{l}\text { Checking that involves active participation, such as } \\
\text { special measurements }\end{array}$ & 0.01 \\
\hline Forms Reconciliation & $\begin{array}{l}\text { Special short-term, one-of-a-kind checking with } \\
\text { alerting factors }\end{array}$ & 0.05 \\
\hline Process Call & $\begin{array}{l}\text { Special short-term, one-of-a-kind checking with } \\
\text { alerting factors }\end{array}$ & 0.05 \\
\hline Material Request & Checking routine tasks using written materials & 0.10 \\
\hline Material Transfer & $\begin{array}{l}\text { Checking by reader/checker of the task performer in } \\
\text { a two-man team, or checking by a second checker, } \\
\text { routine task }\end{array}$ & 0.50 \\
\hline Product Storage & $\begin{array}{l}\text { Checking by reader/checker of the task performer in } \\
\text { a two-man team, or checking by a second checker, } \\
\text { routine task }\end{array}$ & 0.50 \\
\hline Daily Administrative Check & Checking routine tasks using written materials & 0.10 \\
\hline Physical Inventory & $\begin{array}{l}\text { Checking that involves active participation, such as } \\
\text { special measurements }\end{array}$ & 0.01 \\
\hline Inventory Audit & $\begin{array}{l}\text { Checking that involves active participation, such as } \\
\text { special measurements }\end{array}$ & 0.01 \\
\hline
\end{tabular}

Table 2: $\quad$ Frequencies of key administrative MC\&A activities (representative)

\begin{tabular}{|l|c|}
\hline $\begin{array}{l}\text { MC\&A Activity } \\
\text { (Examples of Key } \\
\text { Administrative Controls) }\end{array}$ & $\begin{array}{c}\text { Activity } \\
\text { Frequency } \\
\text { (days) }\end{array}$ \\
\hline Plan of the Day & 1 \\
\hline Daily Administrative Check & 1 \\
\hline Forms Reconciliation & 3 \\
\hline Process Call & 15 \\
\hline Physical Inventory & 30 \\
\hline Inventory Audit & 365 \\
\hline
\end{tabular}

important to consider and understand the dependence between the recurrences of the same activity or between the occurrences of two different activities and whether they are performed by the same or different persons. Dependence is a characteristic used in HRA 
methods to consider how the success or failure of a subsequent task depends on the success or failure of the immediately preceding task.

The failure to address the issue of dependence "may lead to an optimistic assessment of joint HEPs for NPP tasks" [64, p. 10-1]. One method for assessing dependence is a positive dependence model for estimating conditional probabilities for two tasks. Positive dependence implies a positive relationship between events, that is “...failure on the first task increases the probability of failure on the second task" [64, p. 10-4]. The positive dependence model can be applied in situations where actual data on conditional probabilities of success or failure in the performance of tasks is not available.

Equation 15 provides the failure equation that is used to calculate conditional probabilities of failure on Task $M$ given failure on the previous Task $M-1$ for different levels of dependence. The general formulation for the failure equation is:

$$
P\left(F_{M} \mid F_{M-1}\right)=\frac{1+a P_{M-1}}{a+1}
$$

where $a$ ranges from 0 to $\infty$. Values of $a$ equal to $0,1,6,19$, and correspond, respectively, to points of complete, high, moderate, low, and zero positive dependence [64, Equations 10-14 through 10-18].

To explore the dependence that may be generally associated with recurring MC\&A activities, the failure equation for the positive dependence model from Swain and Guttmann [64] was applied for one MC\&A activity that occurs once per day over a 30day period. Figures 10,11 , and 12 show how the daily probability of MC\&A detection varies across five different levels of dependence for a low (0.02), medium (0.50), and high (0.99) initial probability of detection (complement of a BHEP for a type of NPP operation associated with a specific MC\&A activity). These plots demonstrate how, in most cases of human performance, it is expected that when a person performs a recurring 
activity, if he or she does not detect an anomaly in the first one or two opportunities, then the likelihood that the anomaly will be detected will decrease significantly for subsequent opportunities. Generally, with recurring activities, each subsequent opportunity has a decreasing likelihood of successfully detecting an anomaly given that the previous opportunity has failed. With no dependence between recurring MC\&A activities (for example, a different person performing the operation for each recurrence), the initial probability of detection can be maintained over the 30-day timeline. The decrease in probability of detection for each subsequent recurrence of the same activity or of two activities, however, will vary with the level of dependence between the recurrences of the activities, as shown in Figures 10, 11, and 12. The plots differ only in the scale on the yaxis, which reflects the low, medium and high values, respectively, for the initial probability of detection $(0.02,0.50$, and 0.99$)$.

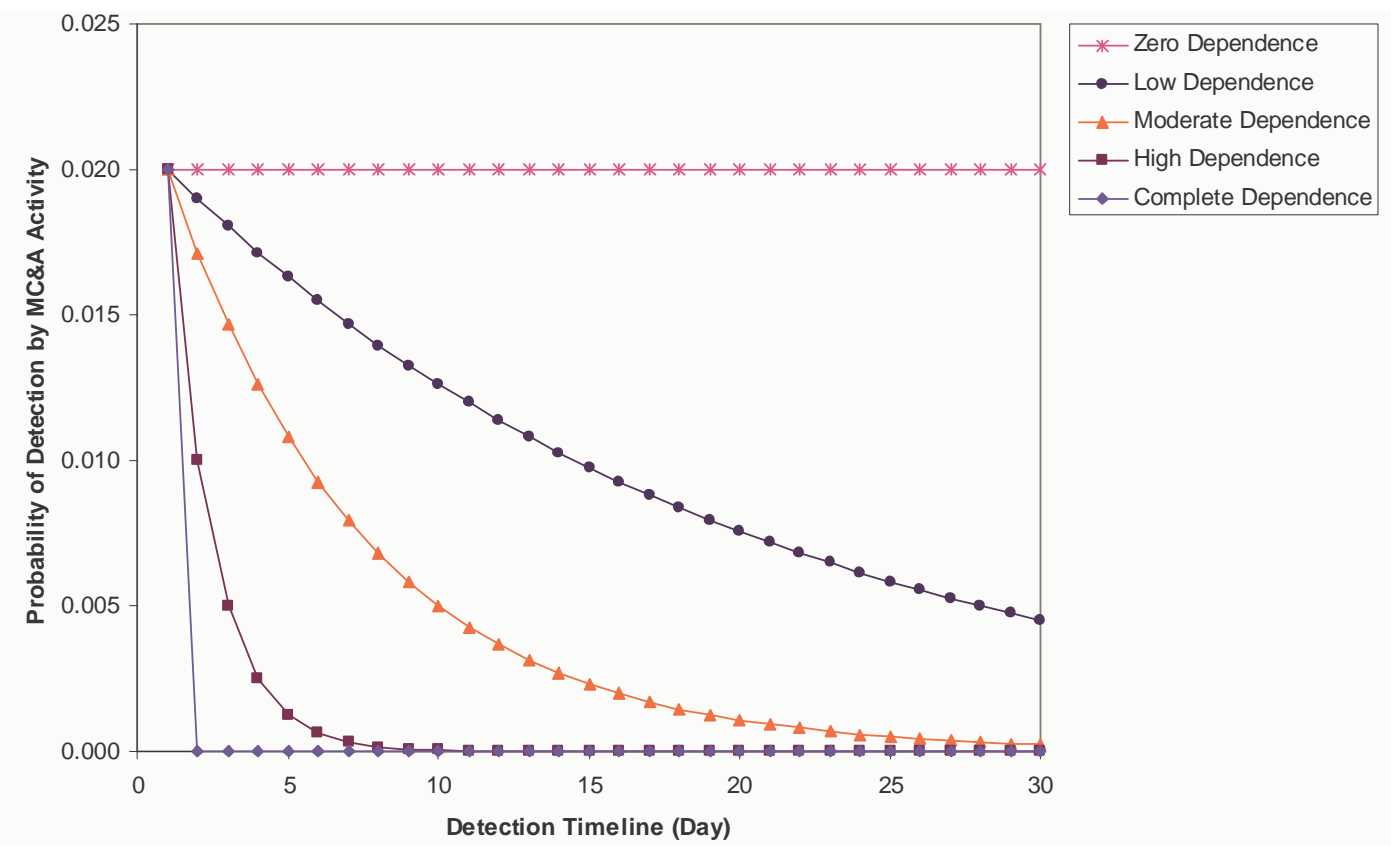

Figure 10: Daily probability of detection over a 30-day period for one MC\&A activity performed once a day based on a BHEP of 0.98 , or an initial probability of detection of 0.02 , for five different levels of dependence. 


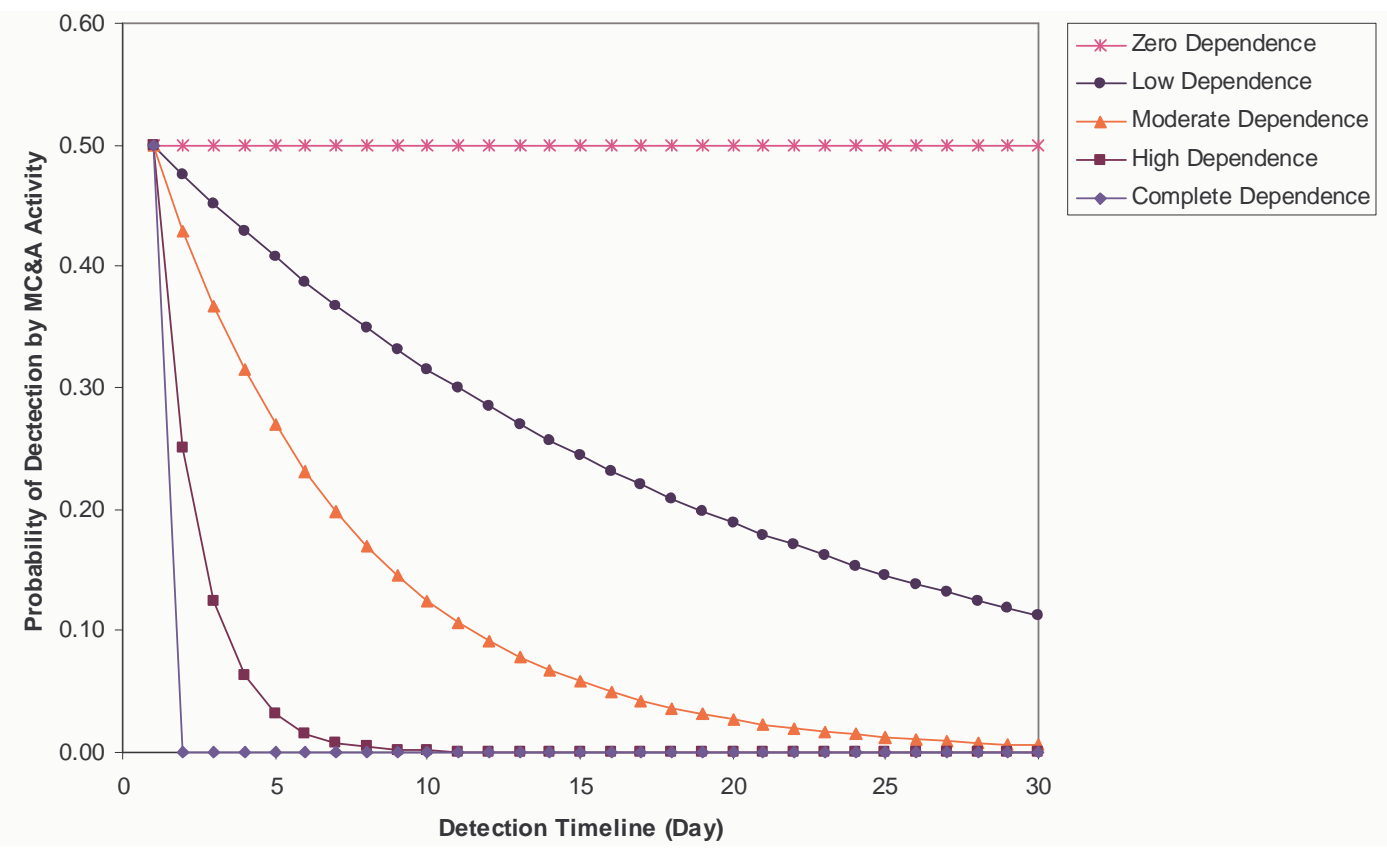

Figure 11: Daily probability of detection over a 30-day period for one MC\&A activity performed once a day based on a BHEP of 0.50 , or an initial probability of detection of 0.50 , for five different levels of dependence.

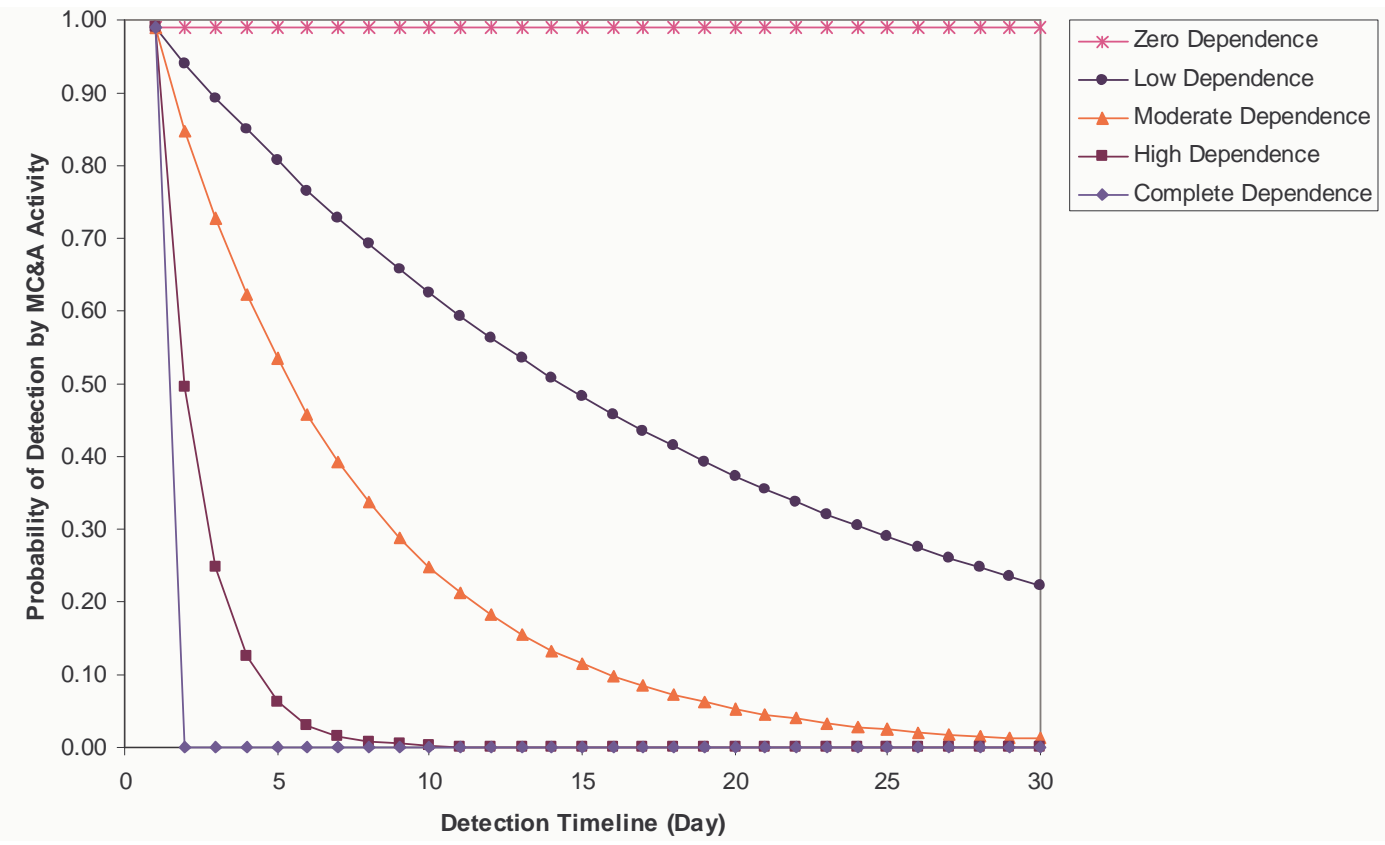

Figure 12: Daily probability of detection over a 30-day period for one MC\&A activity performed once a day based on a BHEP of 0.01 , or an initial probability of detection of 0.99 , for the five different levels of dependence. 


\subsection{TIMELY DETECTION}

The existing path analysis methodology evaluates the PPS for a facility on the basis of detection, delay and response timelines using probabilistic analysis of adversary paths to determine a quantitative probabilistic measure of timely detection. The path analysis methodology calculates the probability $P_{E}$ that the PPS achieves timely detection and is effective in defeating an attack by an outside adversary. This work has developed several elements to provide a probabilistic basis for extending the existing path analysis methodology to incorporate timely MC\&A detection.

MC\&A activities contribute to the effectiveness of the facility protection system by providing alerts that material may be missing. While timely detection for a PPS depends on detection, delay and response that interrupts and neutralizes an attack from an outside adversary, timely detection for MC\&A activities depends on detecting that material is not where it should be and providing an alert. The mathematics for probabilistic convolution provide a basis to determine the probability that an MC\&A alert (detection) causes the Facility to transition to the "Searching for Missing Material" state before the insider moves the material past a given physical protection layer. The effectiveness of MC\&A activities can be determined by convolving the probability distributions for the MC\&A detection timeline with the insider theft timeline to determine the probability that detection occurs before the theft of material can be completed.

\subsubsection{Formulation of Timely MC\&A Detection}

In demonstrating the application of HRA methods for determining a probability of detection for MC\&A activities (Section 3.5.2), only the daily MC\&A detection timeline, specifically for a 30-day scenario, was described without considering the insider adversary theft stages. To determine timely detection, the MC\&A detection timeline must be convolved with the insider adversary theft timeline. MC\&A activities provide 
recurring opportunities to detect that material is "missing" such that the facility state transition occurs from normal state to alert state. Because MC\&A activities are usually discrete observations, discrete mathematics and discrete probability distributions are appropriate. Because the frequency of recurrence for MC\&A activities (Table 2) is determined in days, this formulation used one day as the discretization time step. Other discretization time steps could also be used (if appropriate) based on the frequency of MC\&A activities or theft opportunities. If material is detected as missing on day $n$ and the material has not been removed from the facility before day $n$, then detection will be timely. To formulate the probability of timely detection by MC\&A activities, $P_{D \text {, Timely }}$ is the overall cumulative daily probability of detection over the scenario timeline of $N$ days:

$$
P_{D, \text { Timely }}=\sum_{n=1}^{N} P_{D, \text { Timely }, n}
$$

This is the sum of MC\&A detection that occurs exactly on day $n$ and is timely, that is, detection happens before the insider moves the material out of a physical protection layer. $P_{D, \text { Timely }, n}$, the probability of timely detection on a given day $n$, is defined as:

$$
P_{D, \text { Timely }, n}=P_{D E n} \times P_{N T n}
$$

where:

$P_{D E n} \quad=$ the probability that the facility detects material is missing on exactly day $n$

$P_{N T n} \quad=$ the probability that the material has not been removed from the facility before day $n$

$P_{N T n}$ is the complementary cumulative probability that the theft occurred on day $n, P_{T n}$ :

$$
P_{N T n}=1-\sum_{i=1}^{n-1} P_{T i}
$$


$P_{T n}$ is the daily probability of theft and is determined from the theft opportunity timeline. For example, if an insider has an opportunity to take material once a day over a 30-day time period, then

$$
P_{T n}=\frac{1}{30}=0.033
$$

$P_{T n}$ is determined for various timeline scenarios based on the type of insider and his or her access to the target material.

Further, because detection on exactly day $n$ implies that the material has not been detected as missing before day $n$ and is detected as missing on day $n, P_{D E n}$ is defined as:

$$
P_{D E n}=P_{D, M C \& A, n} \times P_{N D, n-1}
$$

where:

$P_{D, M C \& A, n}=$ the probability of detection for the MC\&A activities on the $n$th day

$P_{N D, n-1}=$ the probability that the material has not been detected as missing before day $n$

The detection probabilities for MC\&A activities can be determined as described in Section 3.6.2 by characterizing individual activities as associated NPP operations and defining applicable BHEPs and dependency relationships. The MC\&A detection probabilities are the complements of the BHEPs. An MC\&A detection timeline for a given scenario is defined as the set of MC\&A activities that are performed on a day-today basis.

$P_{N D, n-1}$, the probability that the material has not been detected as missing before day $n$, is defined as:

$$
P_{N D, n-1}=1-P_{D<n}
$$


$P_{D<n}$ is the cumulative probability that the facility detects material is missing (cumulative $\left.P_{D E n}\right)$ up to day $n-1$ :

$$
P_{D, n}=\sum_{i=1}^{n-1} P_{D E i}
$$

Thus, combining Equations 16 through 22 leads to:

$$
P_{D, \text { Timely }}=\sum_{n=1}^{N} P_{D, M C \& A, n} \times\left(1-\sum_{i=1}^{n-1} P_{D E i}\right) \times\left(1-\sum_{i=1}^{n-1} P_{T i}\right)
$$

\subsubsection{Example Calculation of Timely MC\&A Detection}

Table 3 provides the values for each of the probabilistic parameters required to calculate the probability of timely detection by one MC\&A activity performed once a day in one physical protection layer over a 30-day time period. In this scenario, the insider adversary's opportunity to remove target material occurs once every day, and the adversary will decide during this time period which day will be most advantageous to remove the material from this physical protection layer. For this scenario, then, the insider theft opportunity timeline is defined as a uniform distribution function, so the daily probability of theft, $\mathrm{P}_{\mathrm{Tn}}$, is:

$$
P_{T n}=\frac{1}{30}=0.033
$$

Column 1 of Table 3 is the day, $n$. Column 2 has the daily values for $P_{N T n}$, the probability that the material has not been removed from the facility before day $n$, and is calculated as the complementary cumulative probability that the theft occurred on day $n$. For the uniform insider theft opportunity timeline, this calculation is:

$$
P_{N T n}=1-\sum_{i=1}^{n-1} P_{T i}=1-\frac{(n-1)}{30}
$$


Table 3: Calculation of timely detection over a 30-day scenario for a uniform insider theft timeline and one MC\&A activity performed once a day based on an initial probability of detection of 0.02 , for a moderate level of dependence

\begin{tabular}{|c|c|c|c|c|c|c|}
\hline $\begin{array}{l}\text { Day } \\
\text { (n) }\end{array}$ & $\mathbf{P}_{\mathrm{NTn}}$ & $\mathbf{P}_{\mathrm{D}, \mathrm{MC} \& \mathrm{~A}, \mathrm{n}}$ & $\mathbf{P}_{\mathrm{ND}, \mathrm{n}-1}$ & $\mathbf{P}_{\text {DEn }}$ & $\mathbf{P}_{\mathrm{D}<\mathbf{n}}$ & $\begin{array}{c}\mathbf{P}_{\mathrm{D}, \text { Timely,n }} \\
\left(\mathbf{P}_{\mathrm{DEn}} * \mathbf{P}_{\mathrm{NTn}}\right)\end{array}$ \\
\hline 1 & 1.000 & 0.020 & 1.000 & 0.020 & 0.020 & 0.020 \\
\hline 2 & 0.967 & 0.017 & 0.980 & 0.017 & 0.037 & 0.016 \\
\hline 3 & 0.933 & 0.015 & 0.963 & 0.014 & 0.051 & 0.013 \\
\hline 4 & 0.900 & 0.013 & 0.949 & 0.012 & 0.063 & 0.011 \\
\hline 5 & 0.867 & 0.011 & 0.937 & 0.010 & 0.073 & 0.009 \\
\hline 6 & 0.833 & 0.009 & 0.927 & 0.009 & 0.082 & 0.007 \\
\hline 7 & 0.800 & 0.008 & 0.918 & 0.007 & 0.089 & 0.006 \\
\hline 8 & 0.767 & 0.007 & 0.911 & 0.006 & 0.095 & 0.005 \\
\hline 9 & 0.733 & 0.006 & 0.905 & 0.005 & 0.100 & 0.004 \\
\hline 10 & 0.700 & 0.005 & 0.900 & 0.004 & 0.105 & 0.003 \\
\hline 11 & 0.667 & 0.004 & 0.895 & 0.004 & 0.109 & 0.003 \\
\hline 12 & 0.633 & 0.004 & 0.891 & 0.003 & 0.112 & 0.002 \\
\hline 13 & 0.600 & 0.003 & 0.888 & 0.003 & 0.115 & 0.002 \\
\hline 14 & 0.567 & 0.003 & 0.885 & 0.002 & 0.117 & 0.001 \\
\hline 15 & 0.533 & 0.002 & 0.883 & 0.002 & 0.119 & 0.001 \\
\hline 16 & 0.500 & 0.002 & 0.881 & 0.002 & 0.121 & 0.001 \\
\hline 17 & 0.467 & 0.002 & 0.879 & 0.001 & 0.122 & 0.001 \\
\hline 18 & 0.433 & 0.001 & 0.878 & 0.001 & 0.124 & 0.001 \\
\hline 19 & 0.400 & 0.001 & 0.876 & 0.001 & 0.125 & 4.4E-04 \\
\hline 20 & 0.367 & 0.001 & 0.875 & 0.001 & 0.126 & $3.4 \mathrm{E}-04$ \\
\hline 21 & 0.333 & 0.001 & 0.874 & 0.001 & 0.127 & $2.7 \mathrm{E}-04$ \\
\hline 22 & 0.300 & 0.001 & 0.873 & 0.001 & 0.127 & $2.1 \mathrm{E}-04$ \\
\hline 23 & 0.267 & 0.001 & 0.873 & 0.001 & 0.128 & $1.6 \mathrm{E}-04$ \\
\hline 24 & 0.233 & 0.001 & 0.872 & 0.001 & 0.128 & $1.2 \mathrm{E}-04$ \\
\hline 25 & 0.200 & 4.9E-04 & 0.872 & $4.3 \mathrm{E}-04$ & 0.129 & $8.6 \mathrm{E}-04$ \\
\hline 26 & 0.167 & $4.2 \mathrm{E}-04$ & 0.871 & $3.7 \mathrm{E}-04$ & 0.129 & $6.2 \mathrm{E}-04$ \\
\hline 27 & 0.133 & $3.6 \mathrm{E}-04$ & 0.871 & $3.2 \mathrm{E}-04$ & 0.129 & $4.2 \mathrm{E}-04$ \\
\hline 28 & 0.100 & $3.1 \mathrm{E}-04$ & 0.871 & $2.7 \mathrm{E}-04$ & 0.130 & $2.7 \mathrm{E}-04$ \\
\hline 29 & 0.067 & $2.7 \mathrm{E}-04$ & 0.870 & $2.3 \mathrm{E}-04$ & 0.130 & $1.6 \mathrm{E}-04$ \\
\hline 30 & 0.033 & 2.3E-04 & 0.870 & $2.0 \mathrm{E}-04$ & 0.130 & $6.6 \mathrm{E}-04$ \\
\hline \multicolumn{6}{|c|}{ Cumulative Probability of Timely Detection: } & 0.106 \\
\hline
\end{tabular}

For the example scenario, one MC\&A activity is performed once a day over the 30-day scenario, with a moderate level of dependence between recurrences and a BHEP of 0.98 . Column 3 has the daily MC\&A probability of detection that is calculated from Equation 15 with $a=6$ and an initial probability of detection equal to 0.02 (1-BHEP). As 
expected from the dependence model, the probability of detection decreases for subsequent daily recurrences. Columns 4 through 7 are calculated as described in Section 3.6.1.

The cumulative probability of MC\&A detection is calculated by summing all the daily values in Column 7, and this is the value that is used as the event probability for timely MC\&A detection for the 30-day scenario of one MC\&A activity performed in one physical protection layer once a day and a uniform insider theft timeline. For this scenario, calculations for the probability of timely MC\&A detection were completed for the five different levels of dependence, for a low (0.02), medium (0.50), and high (0.99) initial probability of detection. Figures 13, 15, and 17 show the relationship of daily probability of timely MC\&A detection and dependence for the different initial probabilities of detection, respectively.

Additionally, Figures 14, 16, and 18 show the cumulative probability of detection that could be achieved by one daily MC\&A activity within one physical protection layer over the scenario timeline. The cumulative probability of detection is the value that is used in the ESD for the MC\&A detection events in each physical protection layer to calculate the overall effectiveness for each adversary path scenario.

Note that in each case, while the daily probability of timely detection decreases with time, the cumulative probability of detection improves significantly over the initial individual MC\&A probability of detection. Table 4 summarizes the increase in the cumulative probability of detection after 30 days for each of the initial probabilities of detection and for each of the five dependence levels. Because of the multiple daily detection opportunities, even an MC\&A activity with a low initial probability of detection can achieve a significantly higher cumulative detection probability if the adversary timeline is extended and the dependence between recurrences of activities is 


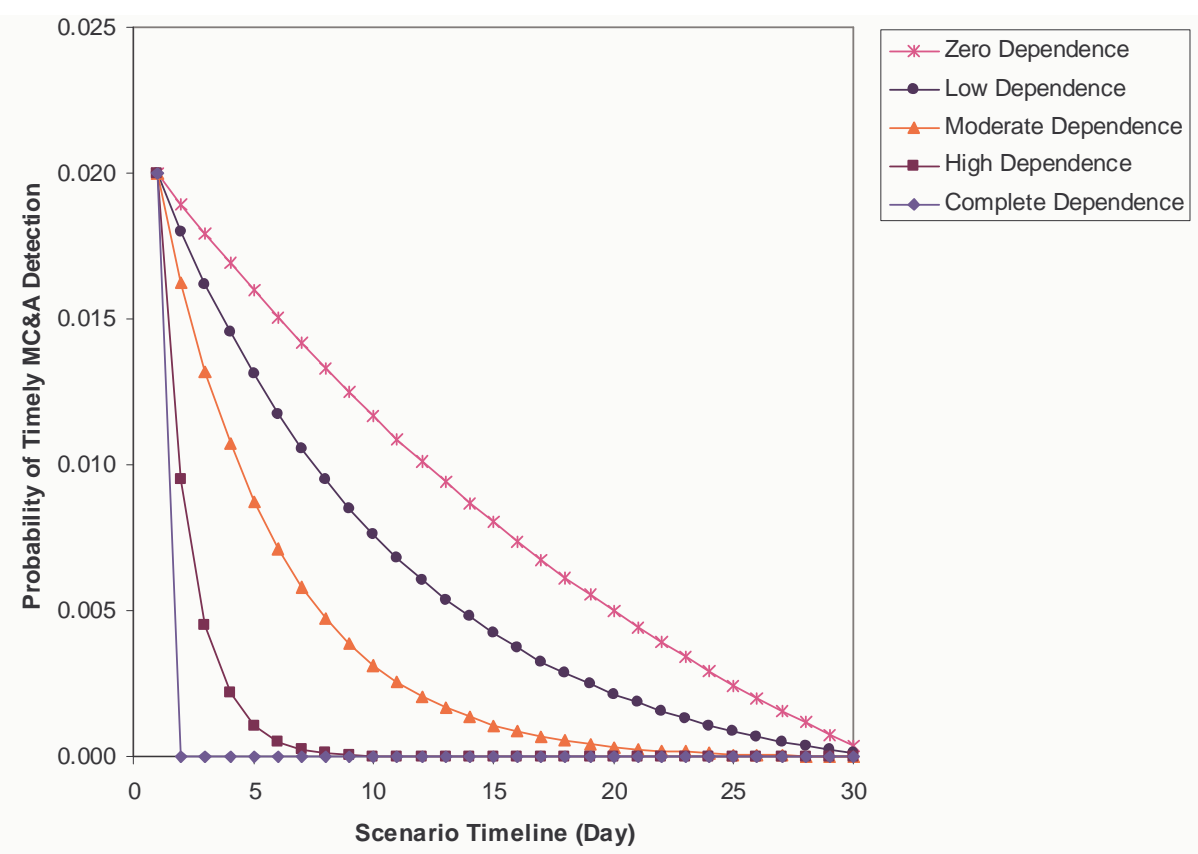

Figure 13: Daily probability of timely detection over a 30-day scenario for one MC\&A activity performed once a day based on a BHEP of 0.98 , or a 0.02 initial probability of detection, for five different levels of dependence.

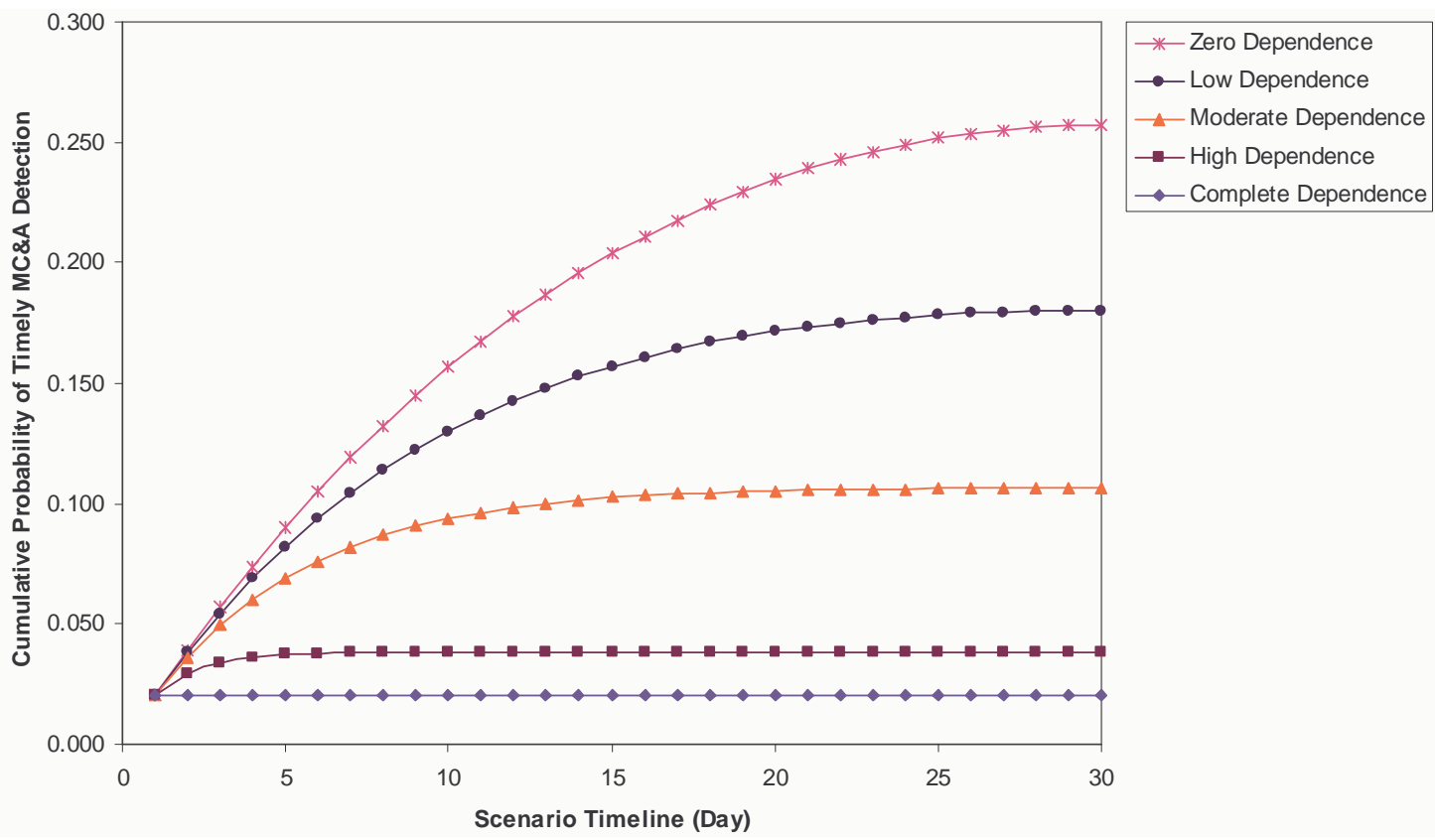

Figure 14: Cumulative probability of timely detection over a 30-day scenario for one MC\&A activity performed once a day based on a BHEP of 0.98 , or a 0.02 initial probability of detection, for five different levels of dependence. 


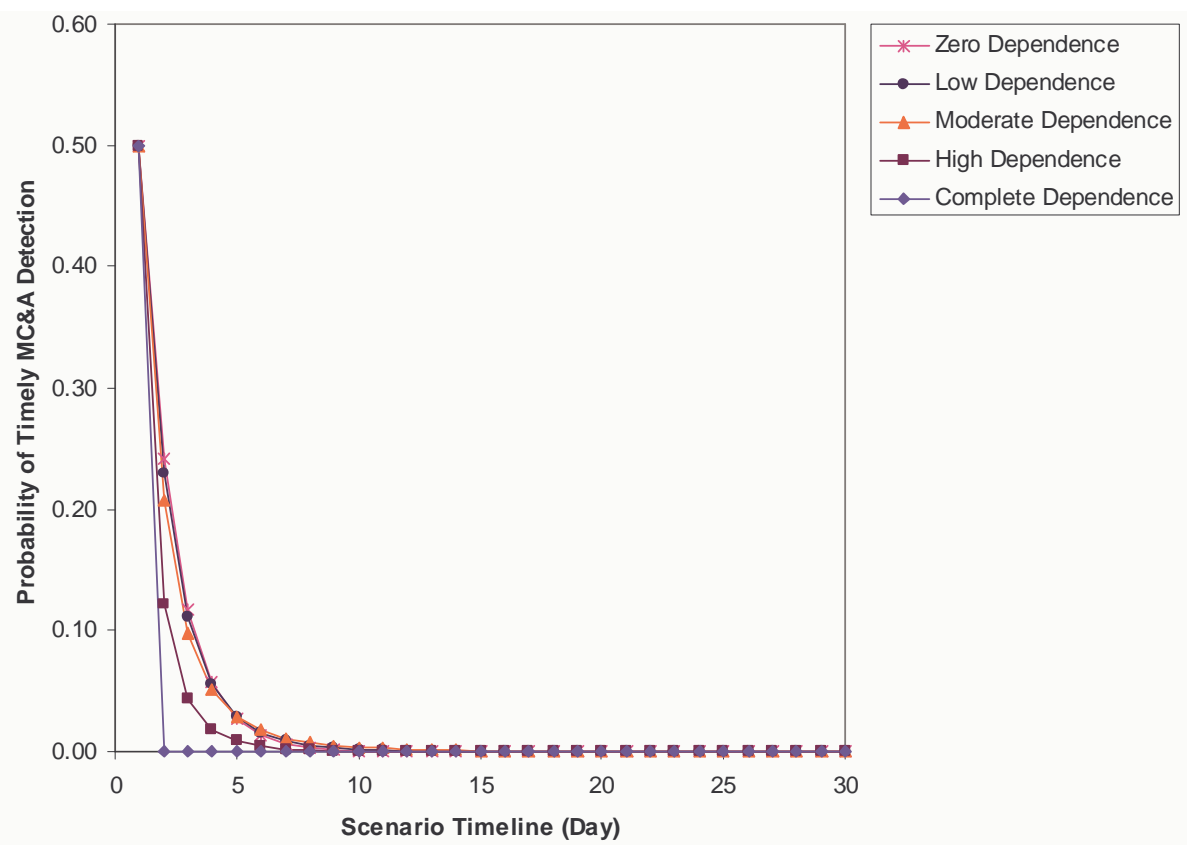

Figure 15: Daily probability of timely detection over a 30-day scenario for one MC\&A activity performed once a day based on a BHEP of 0.50 , or a 0.50 initial probability of detection, for five different levels of dependence.

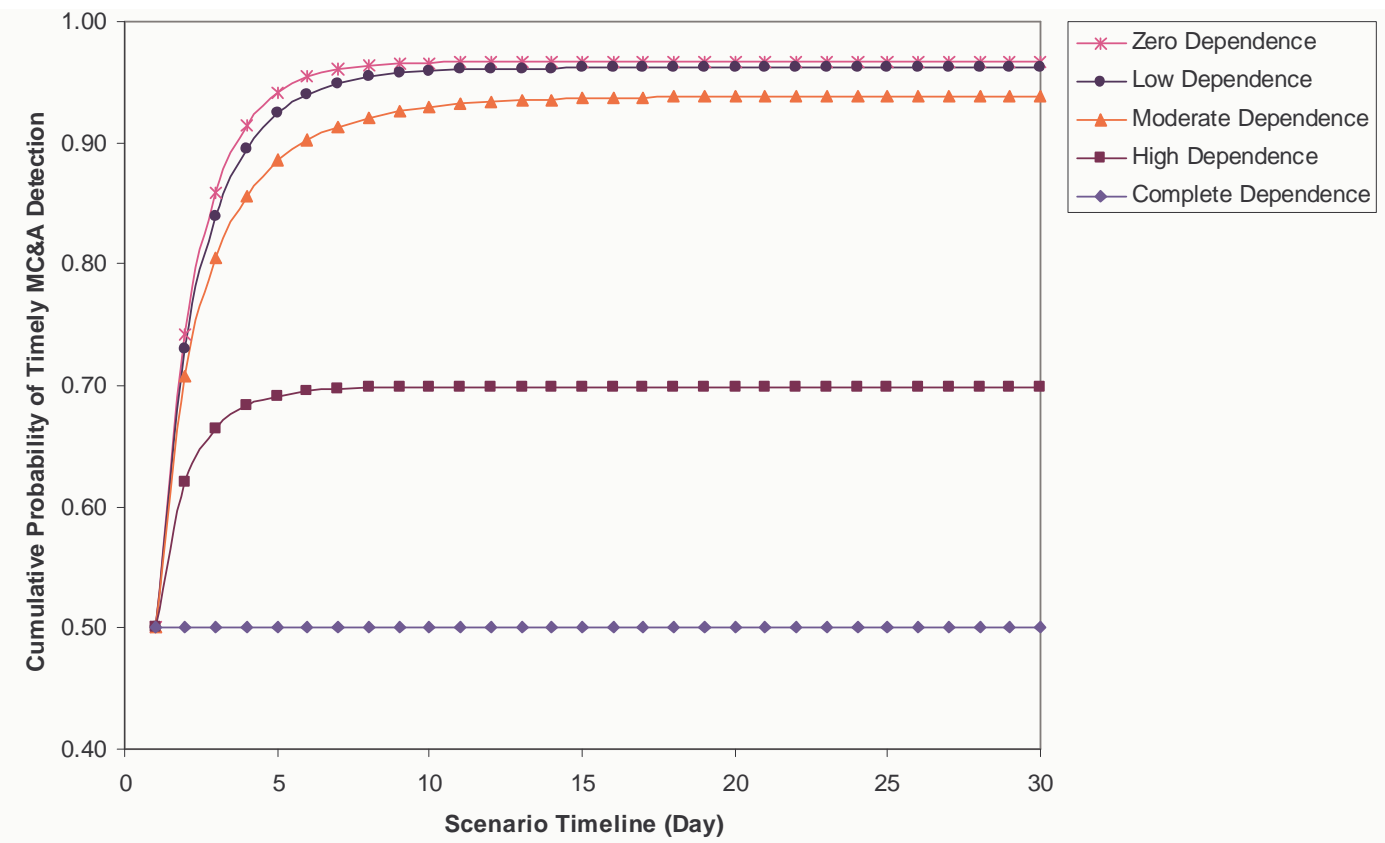

Figure 16: Cumulative probability of timely detection over a 30-day scenario for one MC\&A activity performed once a day based on a BHEP of 0.50 , or a 0.50 initial probability of detection, for five different levels of dependence. 


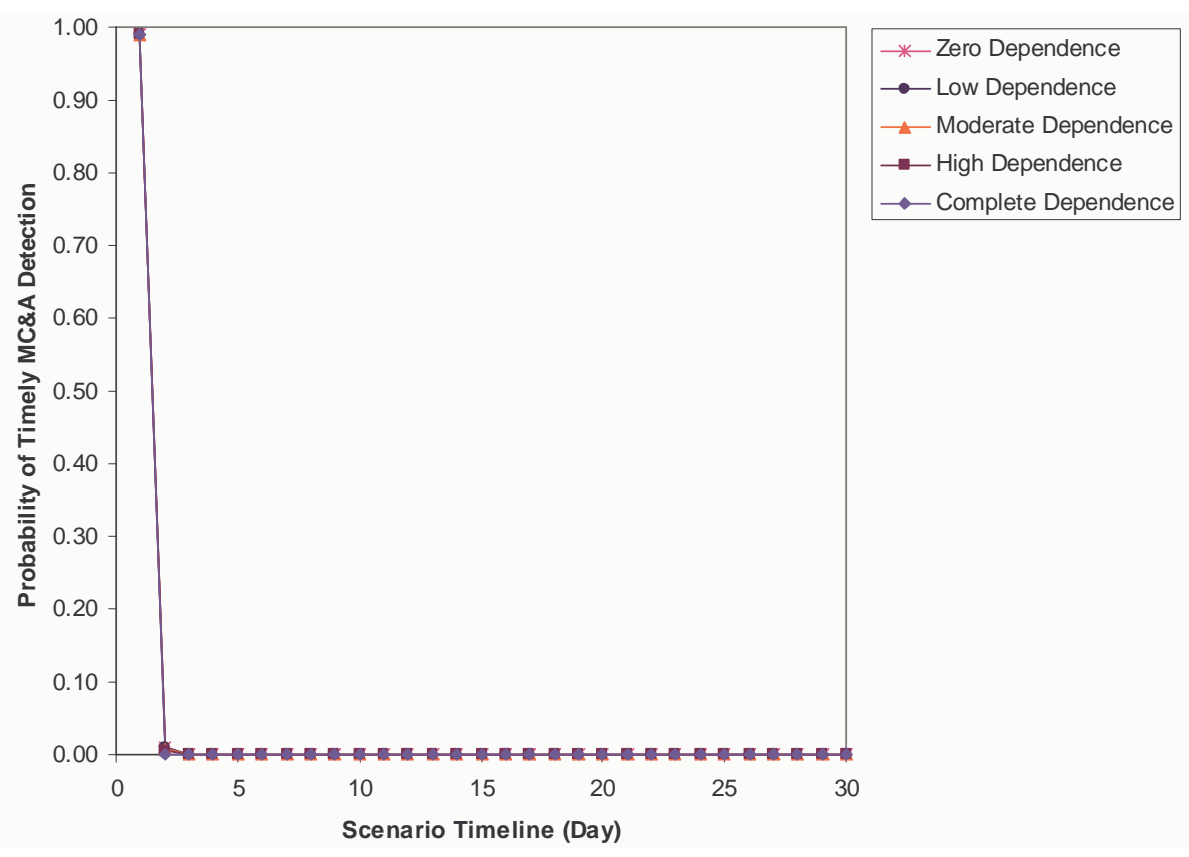

Figure 17: Daily probability of timely detection over a 30-day scenario for one MC\&A activity performed once a day based on a BHEP of 0.01 , or a 0.99 initial probability of detection, for five different levels of dependence.

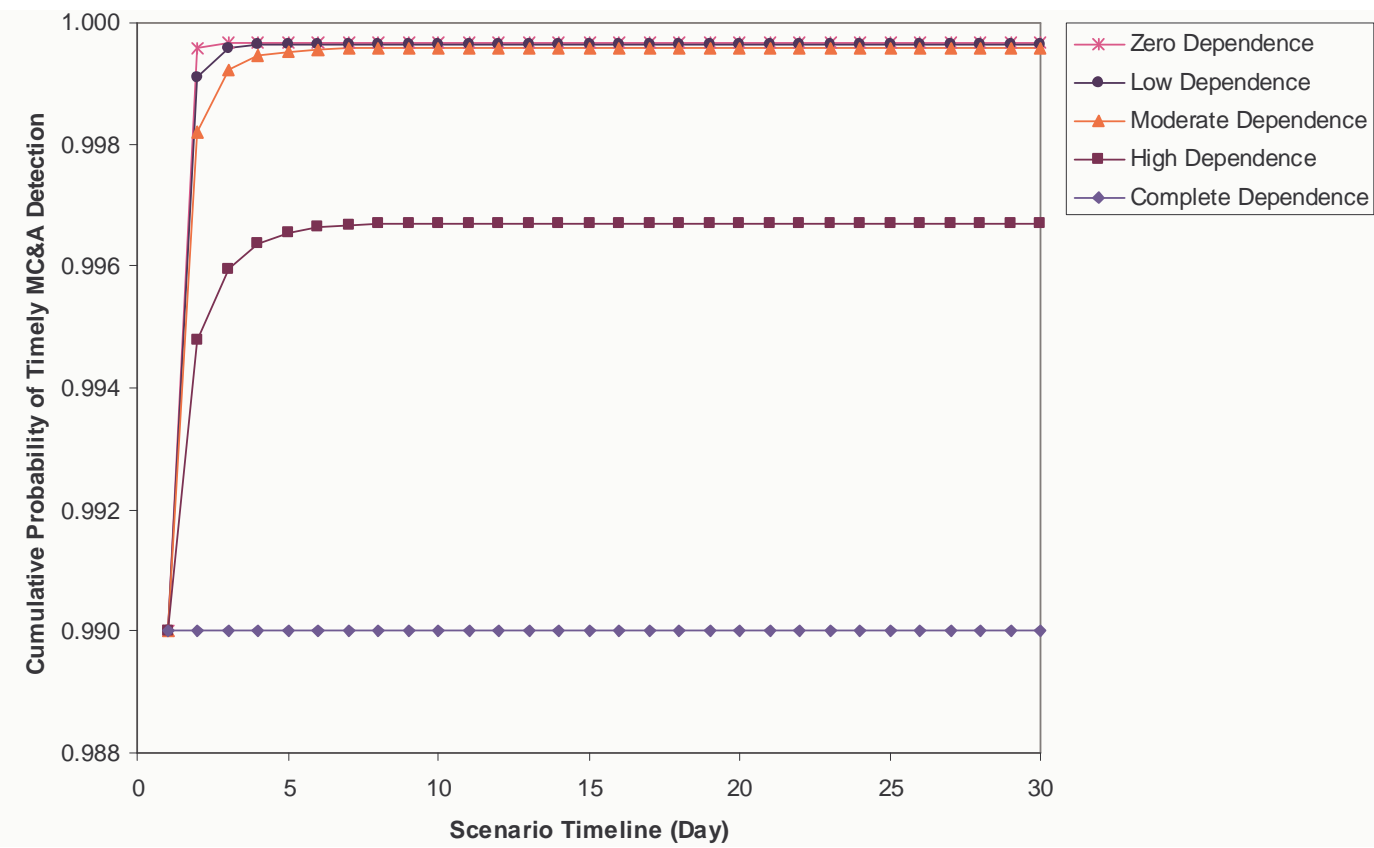

Figure 18: Cumulative probability of timely detection over a 30-day scenario for one MC\&A activity performed once a day based on a BHEP of 0.01 , or a 0.99 initial probability of detection, for five different levels of dependence. 
Table 4: 30-day cumulative probability of MC\&A detection for five dependence levels for low (0.20), medium (0.50), and high (0.99) initial probability of detection

\begin{tabular}{|c|c|c|c|c|c|}
\hline \multirow{2}{*}{$\begin{array}{c}\text { Initial } \\
\text { Probability of } \\
\text { Detection }\end{array}$} & \multicolumn{5}{|c|}{ Level of Dependence } \\
\cline { 2 - 6 } & Complete & High & Moderate & Low & Zero \\
\hline 0.02 & 0.020 & 0.038 & 0.106 & 0.180 & 0.258 \\
\hline 0.50 & 0.500 & 0.699 & 0.939 & 0.963 & 0.967 \\
\hline 0.99 & 0.990 & 0.997 & 0.999 & 0.999 & 0.999 \\
\hline
\end{tabular}

reduced. A more than ten-fold increase $(0.02$ to 0.258$)$ is evident for an activity that has 0.02 initial probability of detection and zero dependence between recurrences of MC\&A observations. It is evident that even one MC\&A activity can provide significant additional detection capabilities; this substantiates the importance of MC\&A activities to protect against suspicious or unauthorized insider activities. This analysis also points to three factors that can be used to "design" MC\&A operations so that theft by a knowledgeable insider is more difficult: developing MC\&A activities that have low BHEPs; reducing the dependency between recurrences of MC\&A activities; and extending the adversary's theft timeline.

\subsection{SumMary OF METHOdS DEVELOPMENT FOR A Single PHYSICAL PROTECTION LAYER}

A focus of this research has been to investigate PRA methods that may be applicable to the development of a probabilistic approach for characterizing MC\&A activities and incorporating an evaluation of the MC\&A component to provide an overall effectiveness measure of the S\&S protection system. The methods in this chapter have applied several PRA techniques and describe the modeling and quantification elements for insider theft and MC\&A characterization. The methods have been demonstrated for the formulation and calculations of timely MC\&A detection by one daily MC\&A event in 
a single physical protection layer for a single theft timeline. The calculation for the insider theft timelines and MC\&A detection becomes more complex as the number of protection layers increases and more MC\&A detection activities are considered. This will be illustrated in analyses that follow in the next chapter for several scenarios with different theft and MC\&A detection timelines and multiple protection layers. 


\section{Chapter 4: Methods for Extended Path Analysis - Daily and Combined MC\&A Detection and Multiple Physical Protection Layers}

To demonstrate the extended path analysis methods beyond a single daily MC\&A activity in a single physical protection layer, additional methods development was required. The calculation for the insider theft timelines and MC\&A detection become more complex as the number of protection layers increases and more MC\&A detection activities are considered. Methods are required for probabilistic inference to determine values of timely MC\&A detection in subsequent physical protection layers and for composite timelines determined from the timelines for each physical protection layer. Calculations were completed for several combinations of timelines for multiple protection layers, with both uniform and variable theft timeline distributions, including a geometric distribution developed using Latin Hypercube Sampling (LHS). In addition, probability of detection calculations for sets of MC\&A activities that occur at different time intervals were completed.

To facilitate this phase of methods development, insider theft scenarios were developed for a hypothetical facility. ${ }^{2}$ An overview of the facility is provided in the next section, followed by a description of and calculations for the insider theft scenarios for the various theft and detection timelines.

\subsection{FACILITY OVERVIEW}

The hypothetical nuclear manufacturing facility (NMF) recycles nuclear material from old dismantled systems into parts for new systems. The dismantled parts are shipped to the NMF where they are broken into chips, recast for machining into new

2 The facility description used here is adapted from one used in training exercises for the Advanced Vulnerability Assessment Overview and Insider Training Courses developed by Sandia National Laboratories [32]. 
parts, then packaged and shipped out to be assembled into new systems. Figure 19 provides an overview of the NMF layout. The NMF includes two MAAs, the main process facility (26) and storage bunker (20), within a PA inside a two-fence perimeter with lights and towers. Two entrances allow vehicles into the PA, one (6) for noncommercial vehicles (mostly the management's personally owned vehicles) and the other (4) for shipments of materials, chemicals, and nuclear material. The processing facility workers park in a lot (3) outside the PA fence and enter on foot through the entry control point (ECP) building (5). A rail entrance on the south of the facility allows for infrequent rail shipments. Along with the process facility and storage bunker, six buildings are inside the PA perimeter, including a cafeteria (16), three support buildings that house

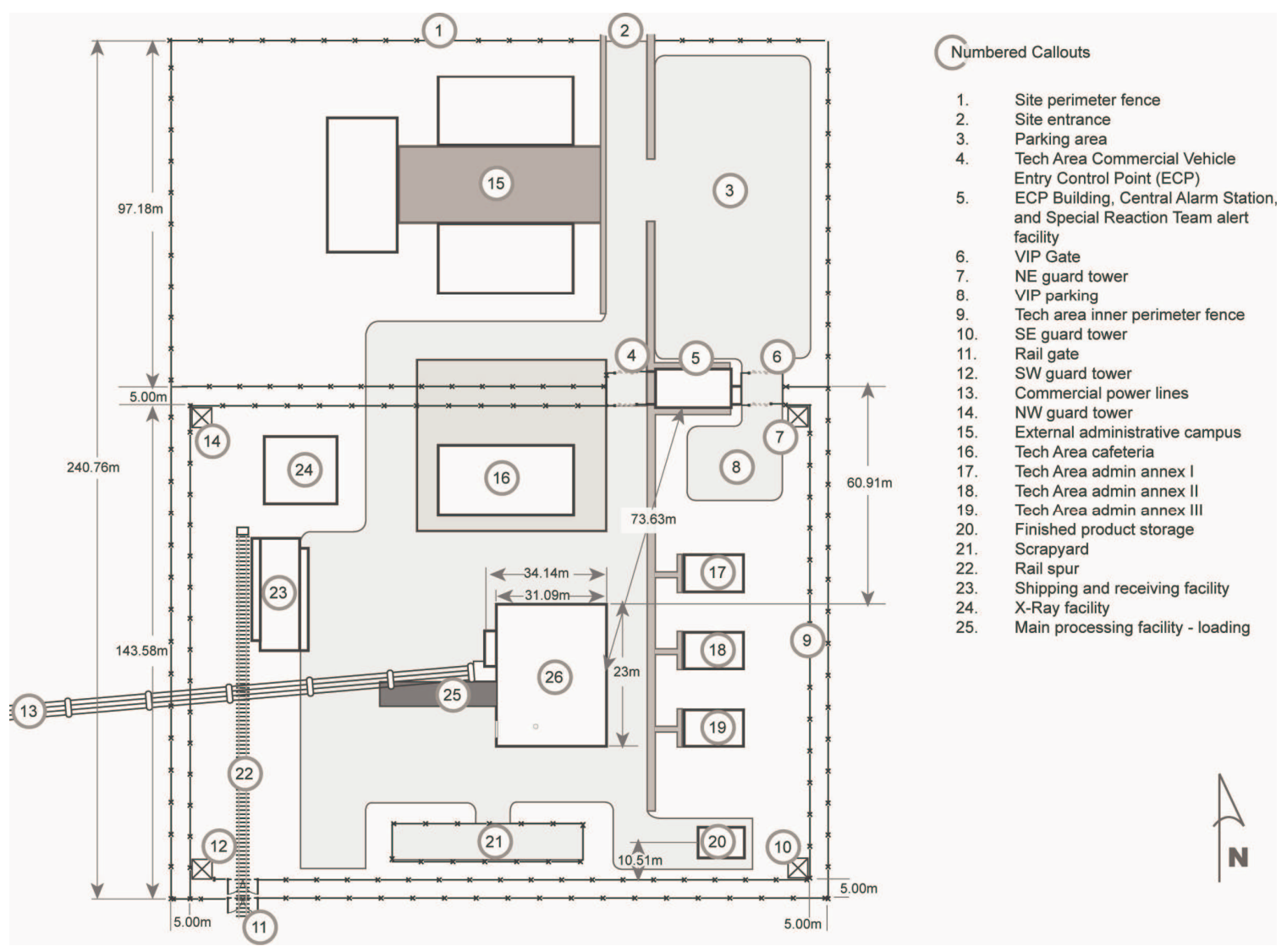

Figure 19: Overview of Nuclear Manufacturing Facility layout [32]. 
offices and light laboratory facilities $(17,18,19)$, a shipping and receiving facility (23), an X-ray facility (24), The PA perimeter consists of two 2.5-m high chain-link fences installed five meters apart; the fences are under observation primarily by the guards in towers at each corner of the PA perimeter $(7,10,12,14)$. Random patrols inside the PA perimeter are conducted by an officer on foot. The guards in the ECP also provide some observation of the PA perimeter within viewing distance of the ECP building.

The outer perimeter of the site is enclosed by a single fence (1). The north fence surrounding the external administrative campus (15) is a 2.5-m high with standard chainlink fabric. The site entrance gate on the north side of the site (2) is unlocked during normal working hours and is locked the rest of the time. The area outside the perimeter has a 20-m cleared zone which is bounded by trees in several locations. The terrain is relatively flat. Random patrols are conducted around the site on a road around the outside of the perimeter.

The process facility near the center of the PA is where the bulk of the processing work is performed. The ECP building straddles the PA perimeter and houses some of the guard force. The ECP into the PA (5) is the main entry point for pedestrian and vehicular traffic where checks are conducted on entry and egress. The outer gate (6) and the ECP are unlocked and open during the normal five-day work hours, which are 7 AM to 6 PM, but locked the rest of the time. Upon entry into the ECP, personnel must show their badge, place their personal items on an X-ray machine belt, and walk through a metal detector. Personnel exiting the processing area enter the ECP through the double doors and pass through a nuclear material monitoring portal. Management and some visitors may enter in personal vehicles through the outer gate to park in the PA. All vehicles entering the PA are subject to search upon entry and pass through a nuclear material 
detector upon exit. Commercial vehicles entering the ECP must present written authorization.

Within the process facility, chipping, melting, casting, and machining operations are performed. The process facility is the primary material area and includes vaults that contain in-process materials (chips, billets, and finished product). The finished products normally weigh between 2 and $3 \mathrm{~kg}$ (depending on the particular product being manufactured). In preparation for off-site shipment, products are packaged in shipping containers and moved to the bunker for storage pending shipment.

The storage bunker is used for storage of nuclear material shipped in for recycling and for storage of finished products packed and ready to ship. The material for recycling is received in approved shipping containers that weigh $100 \mathrm{~kg}$. The product containers weigh 50 or $100 \mathrm{~kg}$ (depending on the type). The 100-kg product containers are essentially the same as the containers for received material. The $50-\mathrm{kg}$ containers are designed to fit inside a larger shipping overpack container and are not as robust as the 100-kg container. They are about one half as tall and the lids snap on with three quick release levers. The same type of inner container is used for all items (there will be some variations in shape/size).

The processing area has an extensive material measurement and control system in place, including procedures to receive material from off-site, to transfer material from the storage bunker to processing, to repackage and weigh material in-process, and to move product within the site for X-ray and storage. All measurements and container identification are documented at each process step. This information is sent to the MC\&A recording area where it is examined, stored, and used to derive a weekly book inventory and material balance. In addition, a physical inventory is conducted monthly. 
The personnel at the NMF include managers, shift supervisors, operators, maintenance staff, technician, guards, and administrative support. The Material Control Manager (MCM) is assigned responsibility for technical coordination of the overall MC\&A program and has specific duties associated with receipt of recycling material onto the site, shipments of finished product off-site, and records for materials in the PA outside the processing facility (storage bunker, X-ray, and sampling). The Material Custodian (MC) reports directly to the MCM and has responsibility for materials in the processing facility. Both these positions have a high level of access to materials, equipment and tools in the PA; authority to request, document, and approve material transfers and measurement records; and knowledge about processing and material control operations.

\subsection{BASIC INSIDER THEFT SCENARIO}

The basic scenario used for the demonstration analyses involves theft of feed material or finished product from the storage bunker within the PA and removal through the personal vehicle entrance. The MCM is the insider adversary and has authorized access through the outer gate to park in the PA and to enter to all buildings and areas within the PA. Inventory in the bunker is conducted on a monthly basis, and transfers from the bunker to the processing building occur on a regular basis. In addition, the nuclear material detector on the outer gate into the PA has maintenance scheduled on a monthly basis. While maintenance occurs, use of the nuclear material detector is replaced by a general random vehicle search. The plan is to acquire target material during authorized access at the bunker, conceal it on his person, move it to an office in the laboratory/office building nearest the PA parking area, and then move it to his vehicle to exit the PA when maintenance is occurring on the nuclear material detector on the outer gate. 


\subsection{SCENARIOS FOR ONE DAILY MC\&A DETECTION ACTIVITY AND VARYING TIMELINES}

This set of scenarios will consider varying timelines for the MAA and PA physical protection layers and one daily MC\&A detection activity. The MCM has daily access to the storage bunker. In these scenarios, the MCM's opportunity to remove target material occurs once every day, and this insider will make a decision during a given timeline as to which day will be most advantageous to remove the material from each physical protection layer. His decision to take action is based on his knowledge of when certain operational conditions (material transfers or detector maintenance) might occur and to what extent he can exploit these. Each protection layer considers both PPS and MC\&A detection elements.

\subsubsection{0-Day Timeline for the MAA and for the PA}

This scenario involves a 30-day theft timeline in both the MAA and PA, for a total scenario timeline of 60 days. Inventory is performed once a month in the storage bunker, and because of his access and authority, the MCM knows he has an opportunity to use deceit to hide any inventory discrepancies in the MAA. Material transfers between the bunker and the process building MAA occur on a regular basis, although the MCM may not know specifically when a transfer may occur. This timeline also considers the 30-day window between maintenance of the nuclear material detector at the outer gate. Because the opportunity to remove target material may occur on any given day in both the MAA and PA, for this example the insider theft timelines are defined as uniform discrete distributions for each of these theft stages.

This example tracks the theft and detection for this scenario through an ESD (see Figure 20). The scenario begins with detection of theft by the PPS protection elements in the MAA, Event 1 in the ESD. Because the adversary is an insider with authorized 
access and operational knowledge, it is assumed that he will be able to circumvent the PPS protection elements. The only PPS protection element that may provide detection in this situation is general observation of suspicious or unauthorized activity by guards or other personnel in the area. Garcia [17] discusses how general observation has a very low probability of detection activity, so the probability of detection for this event is estimated to be 0.02 , and its complement of 0.98 is the probability of non-detection.

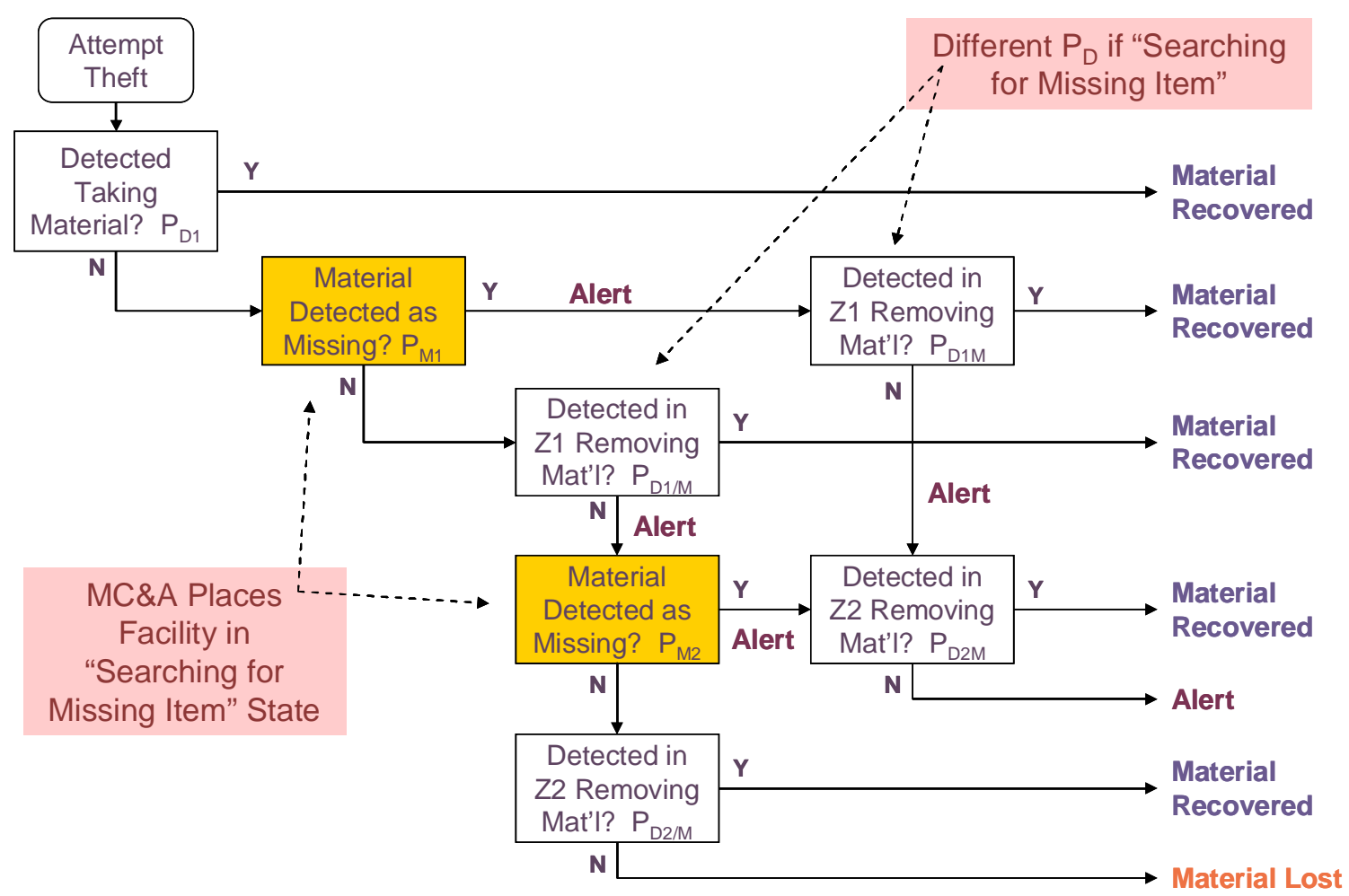

Figure 20. ESD for tracking theft and detection.

Figure 21 illustrates Event 1 in the ESD. If detection occurs and material is recovered, the end state for this event sequence is "Material Recovered," and the overall sequence probability is 0.020 . With no detection for this event, the sequence continues to Event 2 . 


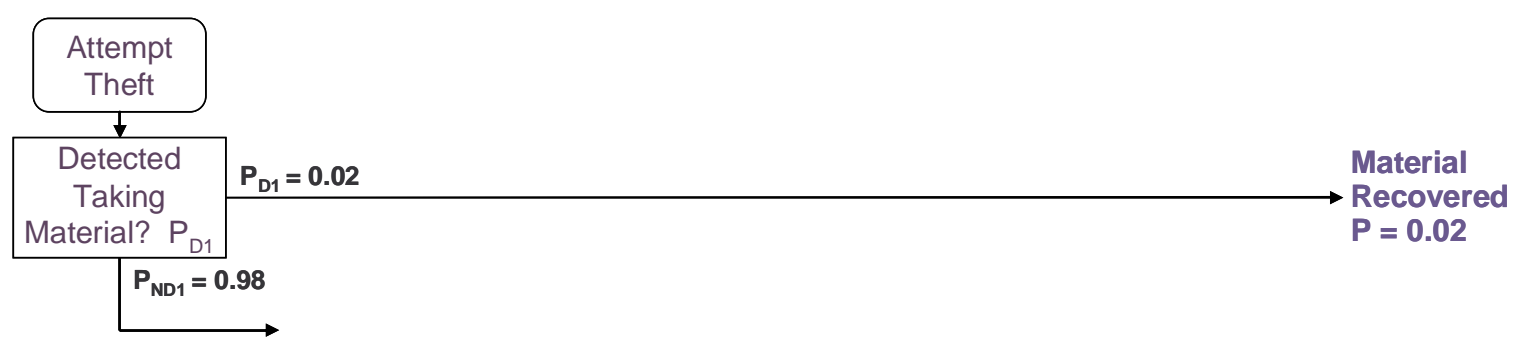

Figure 21: Event 1 of the ESD - Detection of the insider taking the material by the PPS in the MAA

Event 2 is an MC\&A detection that occurs while the stolen material is still in the MAA. This scenario begins with the 30-day scenario calculations described in Section 3.6 for removal of material from the MAA. From the calculations in Section 3.6 for an MC\&A activity with a low initial probability of detection of 0.02 and a moderate level of dependence, the probability of timely MC\&A detection is 0.106 , and the probability of non-detection is 0.894 . If timely MC\&A detection occurs, then the facility moves to an alert state in which it is known that material is not where it should be. Also, in this case, the insider adversary has not been able to remove the material from the MAA into the PA. Figure 22 illustrates the ESD through Event 2 with the possible paths to an alert state or continued normal operations.

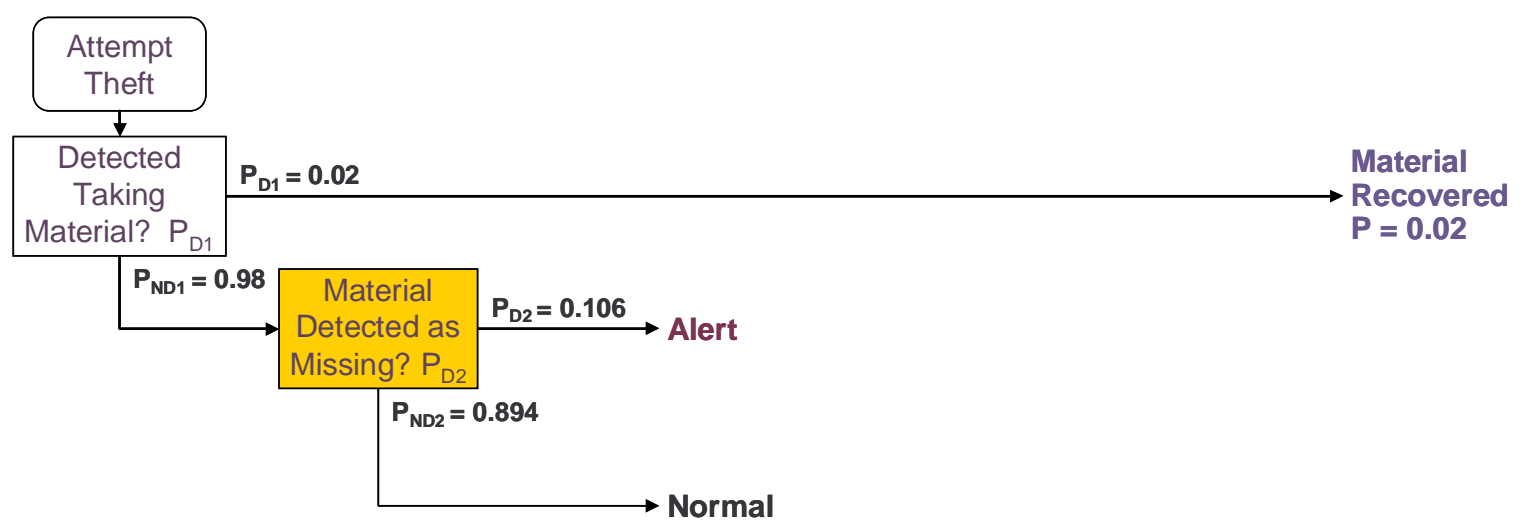

Figure 22: The ESD through Event 2 - Timely MC\&A detection in the MAA 
Events 3 and 4 will be for detection in the PA. Event 3 is for detection by PPS protection elements of material moving into the PA. Two conditions and paths through the ESD are possible here depending on whether the facility is in the alert state or continued normal operations. If no alert occurs, then detection of unauthorized activity again is provided only by general observation and the probability of detection is 0.02 . Once the MCM has taken the target material out of the bunker, he will have to move it across the PA into the laboratory/office building nearest the PA parking lot. If detection occurs, the end state for this event sequence is "Material Recovered," and the overall sequence probability is $0.018(0.98 \times 0.894 \times 0.02)$. With no detection for this event, the sequence continues onto Event 4.

For the second condition for Event 3, when the facility is in an alert state and it is known that material is not where it should be, it is expected that additional efforts will be made throughout the facility to locate the missing material. The probability for detection then can be increased because of these additional efforts. If detection does not occur, the MCM is able to successfully move the material out of the PA, but the facility remains in the alert state. The probability of detection during an alert state is set at 0.50 to reflect increased efforts (significantly greater than relying on general observation) to locate the missing material. If detection occurs, the end state for this event sequence is "Material Recovered," and the overall sequence probability is $0.052(0.98 \times 0.106 \times 0.50)$; otherwise the event sequence skips Event 4 (because MC\&A detection has already occurred) and continues on to Event 5 with the facility in the alert state. Figure 23 illustrates the ESD through Event 3. 


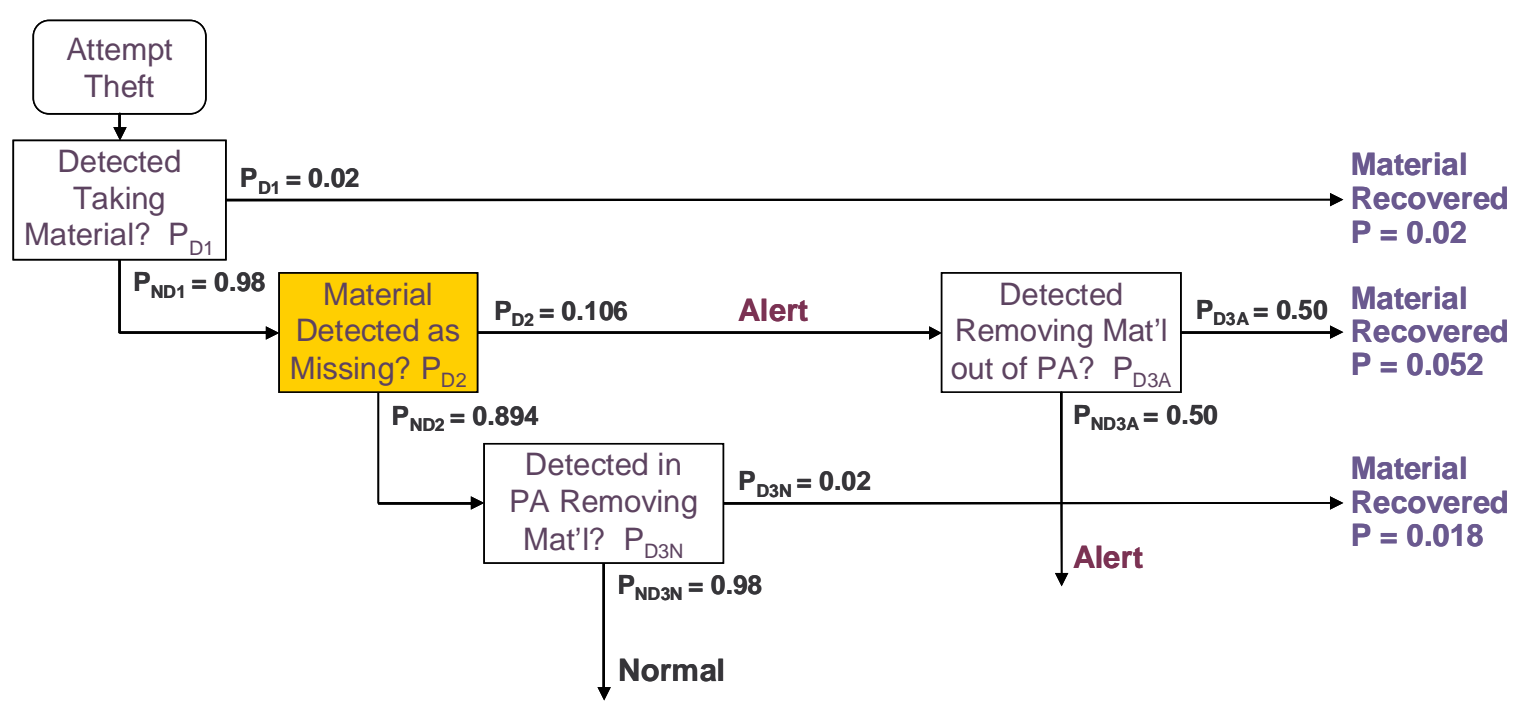

Figure 23: The ESD through Event 3 - Detection of the insider moving the material by the PPS in the PA

Event 4 is MC\&A detection that occurs while material is in the PA. To calculate the probability of timely MC\&A detection in the PA, first the probability of timely MC\&A detection any time before the material leaves the PA during the composite timeline is calculated, $P_{D \text {, Comp. }}$. This value then is used with the probability of timely MC\&A detection in the MAA $\left(P_{D 2}\right)$ to infer the probability of timely MC\&A detection in the PA $\left(P_{D 4}\right)$. The calculation method for probabilistic inference is described later in this section after a discussion of the composite theft timeline during which the MCM can move material from the MAA and then the PA. Once the theft has progressed into the PA, the scenario timeline is the sum of the individual timelines in the MAA and the PA, in this case up to 30 days each for a total scenario timeline of up to 60 days. The theft timeline includes every possible composite timeline over the 60-day duration. For this scenario, the timeline for each physical protection layer ranges from 1 to 30 days, and so $30 \times 30=900$ composite timelines are possible. To determine the probability distribution for theft over the complete 60-day scenario, the two individual uniform distributions have 
to be summed to determine the probability of theft for the possible composite timelines for each day. The individual timelines are independent discrete random variables, $T_{l}$ and $T_{2}$, with uniform distribution functions, and $T_{3}$ is their sum. The distribution function for the composite timeline is determined by convolution of the distribution functions for $T_{1}$ and $T_{2}$, as follows:

$$
P\left(T_{3}=t_{3}\right)=\sum_{t_{1}=1}^{n} P\left(T_{1}=t_{1}\right) \times P\left(T_{2}=t_{2}\right)
$$

where

$t_{2}=t_{3}-t_{1}$ for $T_{3}=T_{1}+T_{2}$.

To calculate timely MC\&A detection with Equation $16, P_{N T n}$, the probability that the material has not been removed from the facility before day $n$ is calculated as the complementary cumulative probability distribution of the composite theft timeline. The MC\&A detection timeline is also determined for the 60-day duration of the theft timeline. The calculation of timely MC\&A detection then follows the same steps outlined for the 30-day scenario in Section 3.6. For an MC\&A activity with an initial probability of detection of 0.20 and a moderate level of dependence, the probability of timely MC\&A detection for the composite timeline is 0.126 . This calculation of timely MC\&A detection considers the total 60-day timeline and is a composite of timely MC\&A detection for both the MAA and the PA. The portion that applies to timely detection in the PA must be inferred from the composite detection and timely detection in the MAA. The method for this probabilistic inference is described as follows. Figure 24 shows a condensed event tree with the two MC\&A detection events, one in the MAA and one in the PA, along with the sequence probabilities for each of the three possible end states, as follows: 


$$
\begin{aligned}
& X=P_{D 2} \\
& Y=\left(1-P_{D 2}\right) \times P_{D 4} \\
& Z=\left(1-P_{D 2}\right) \times\left(1-P_{D 4}\right)
\end{aligned}
$$

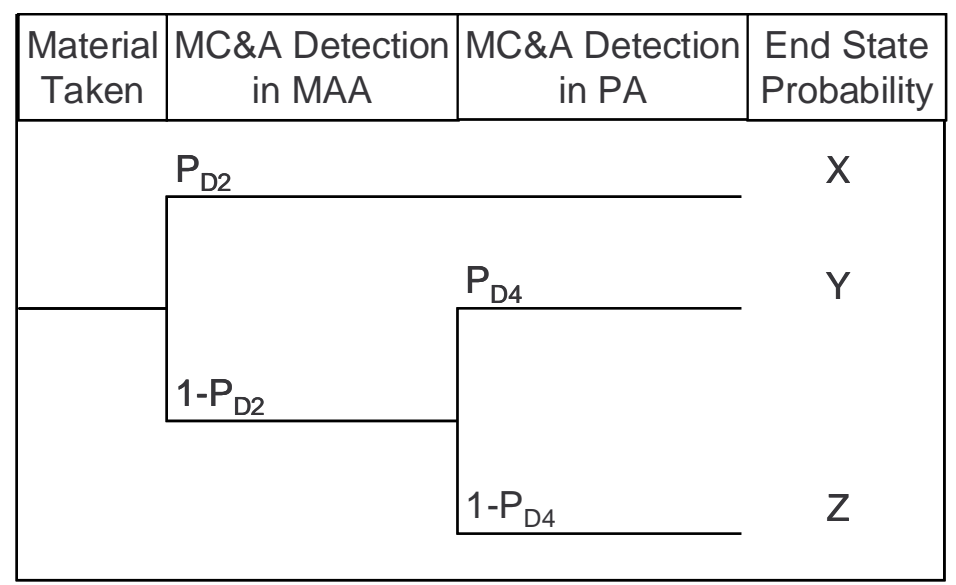

Figure 24: Event tree for MC\&A events in the composite timeline.

The sum of the probabilities for the three end states must equal one. The required value is $P_{D 4}$, which from the sequence probability for $\mathrm{Y}$ is:

$$
P_{D 4}=\frac{Y}{\left(1-P_{D 2}\right)}=\frac{(X+Y)-X}{\left(1-P_{D 2}\right)}=\frac{(X+Y)-P_{D 2}}{\left(1-P_{D 2}\right)}
$$

The value for $(\mathrm{X}+\mathrm{Y})$ is 0.126 and was calculated above as the probability of timely MC\&A detection for the composite 60-day timeline; the value of $P_{D 2}$ was calculated for Event 2. The value

$$
P_{D 4}=\frac{P_{D, \text { Comp }}-P_{D 2}}{\left(1-P_{D 2}\right)}=\frac{0.126-0.106}{(1-0.106)}=0.022
$$

is the value for the probability of timely MC\&A detection for Event 4; the probability of non-detection is 0.978 . If timely $\mathrm{MC} \& \mathrm{~A}$ detection occurs here, then the facility has another opportunity to move to an alert state in which it is known that material is not where it should be. Again, in this case, the insider adversary has not been able to remove 
the material out of the PA. Figure 25 illustrates the ESD through Event 4 with the possible paths to an alert state or continued normal operations.

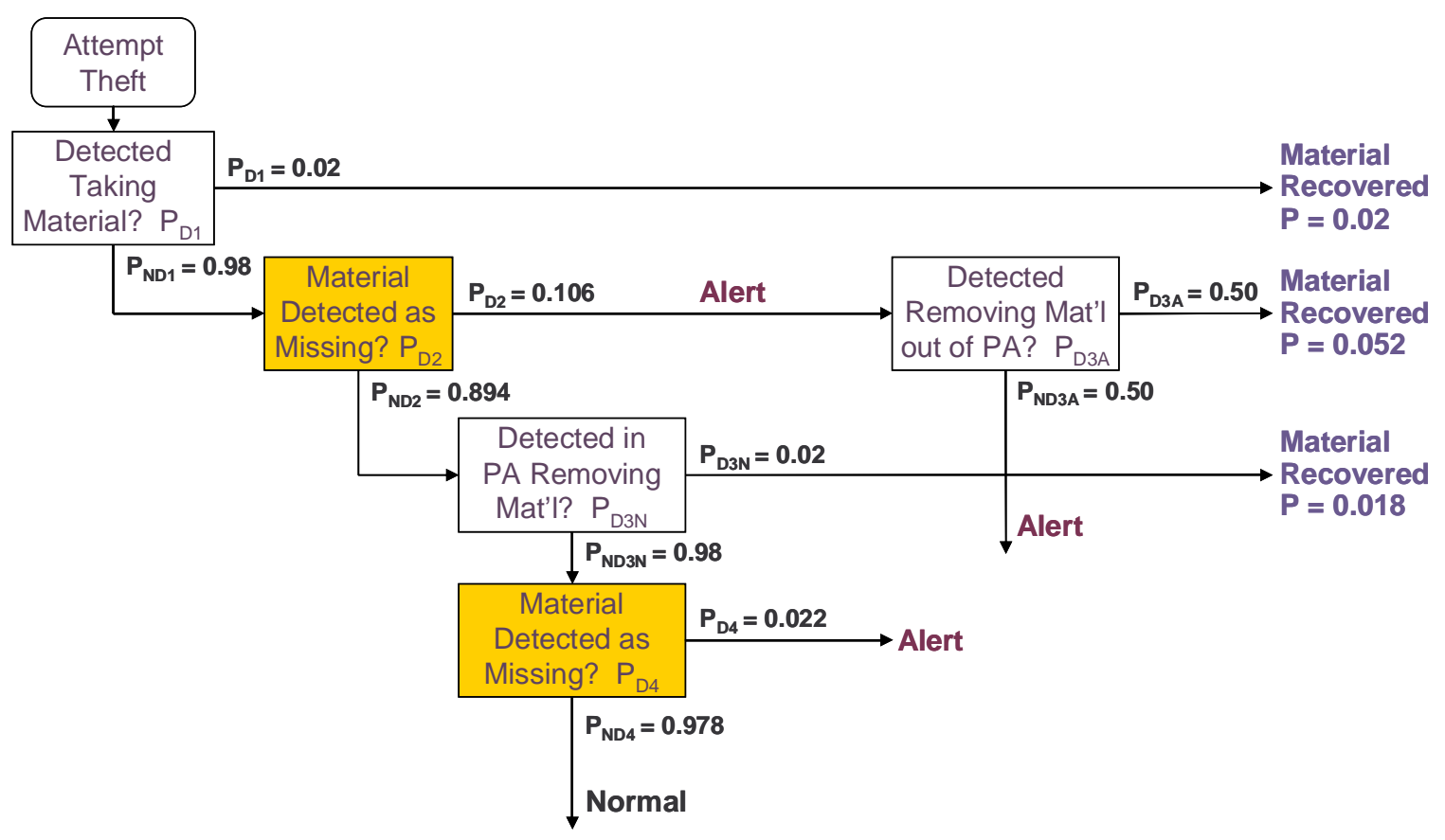

Figure 25: The ESD through Event 4 - Timely MC\&A detection in the PA

Event 5 is for detection by PPS protection elements of material moving out of the PA through the outer gate, which the MCM will plan to do when the nuclear material detector is in maintenance so his vehicle is subject only to a random vehicle search. Similarly to the case for Event 3, two conditions and paths through the ESD are possible here depending on whether the facility is in the alert state or continued normal operations. If no alert occurs, then detection of unauthorized activity again is provided only by detection of the material during a random vehicle search. Once the MCM has moved the target material across the PA into the laboratory/office building nearest the PA parking lot, he will look for an opportunity to take it to his vehicle when the detector at the gate is undergoing maintenance. Again, detection relies on general observation of suspicious or 
unauthorized activity, and the probability of detection is set to 0.02 . The final end states for the two sequences from this event are "Material Recovered," with an overall sequence probability of $0.017(0.98 \times 0.894 \times 0.98 \times 0.978 \times 0.02)$, and "Material Lost" with a sequence probability of $0.823(0.98 \times 0.894 \times 0.98 \times 0.978 \times 0.98)$.

For the second condition for Event 5, when the facility is in an alert state and it is known that material is not where it should be, it is expected that additional efforts will be made throughout the facility to locate the missing material. This is another opportunity to increase the probability for detection because of these additional efforts. The probability of detection during alert is set at 0.50 , again to reflect increased efforts to locate missing material. If detection occurs, the end state for this event sequence is "Material Recovered," and the overall sequence probability is $0.009(0.98 \times 0.894 \times 0.98$ $x 0.022 \times 0.50)$. Otherwise two event sequences end in an "Alert" state: the sequence that continues from Event 3 with a sequence probability of $0.026(0.98 \times 0.106 \times 0.50 \times$ $0.50)$ and the sequence from Event 4 with a sequence probability of $0.009(0.98 \times 0.894 \times$ $0.98 \times 0.022 \times 0.50)$. Figure 26 illustrates the ESD through Event 5.

If detection does not occur and the MCM is able to successfully move the material out of the PA, the facility remains in the alert state. This is an important distinction in terms of information a site has about the status of critical items. The "Material Recovered" end state indicates that the theft was detected or an MC\&A activity alerted the facility that material was not where it should be and that subsequent actions recovered the material before it could be taken out of the facility. The "Material Lost" end state indicates that no MC\&A alert occurred and the facility has no information at the end of the scenario timeline that material is missing - the case of where you do not know what you do not know. An end state of "Alert" indicates that although material may have been successfully removed from the site, the facility knows that material is missing and can 
continue efforts to recover the material, pursue those responsible for the theft and address system vulnerabilities to prevent future theft.

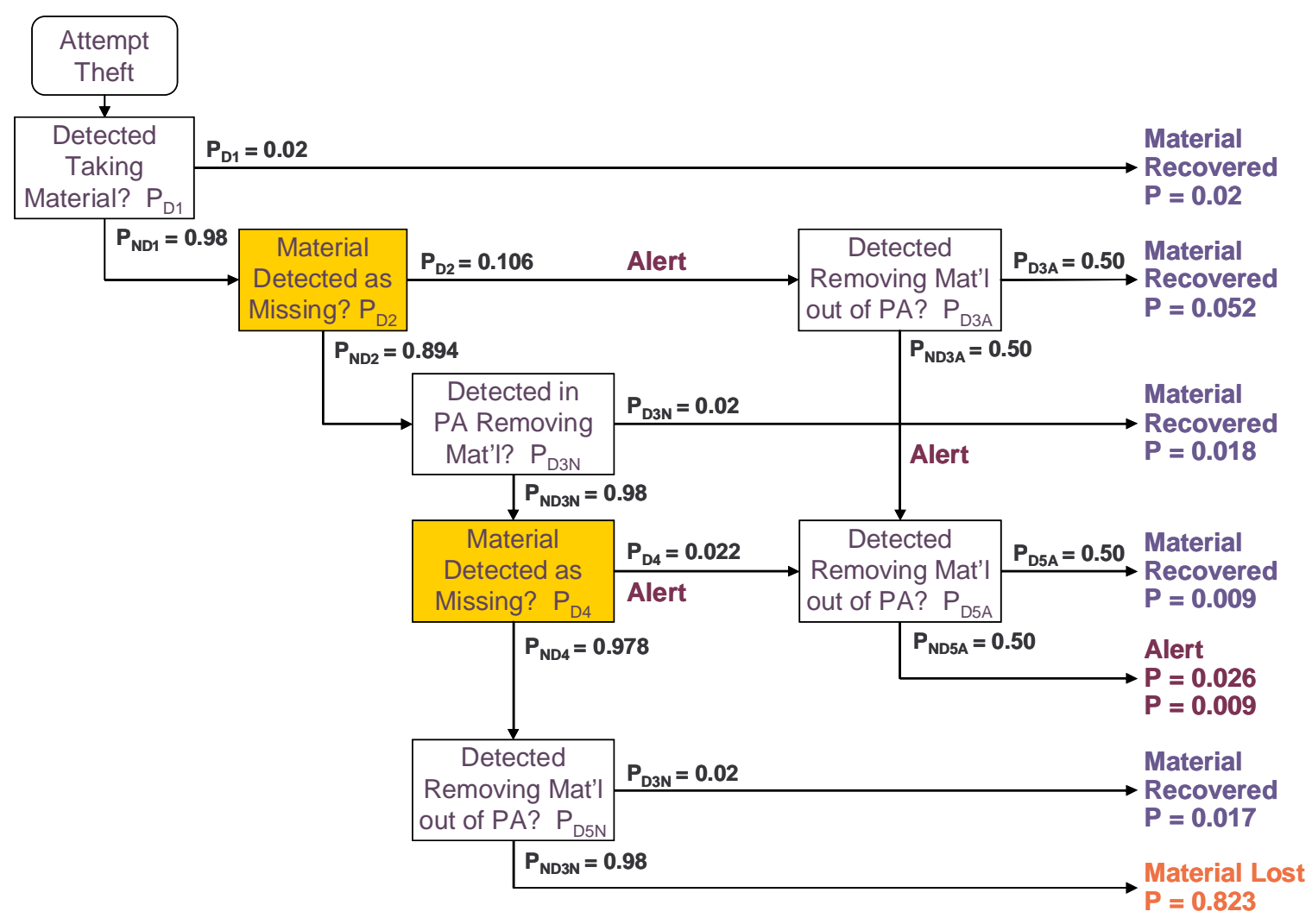

Figure 26: The ESD through Event 5 - Detection by the PPS of the insider moving the material out of the PA

This set of calculations for the 30-day MAA/30-day PA timeline scenario was performed for each level of dependence and the low, middle, and high initial probability of MC\&A detection. Figure 27 shows the event sequence calculations in which Events 2 and 4 represent the timely MC\&A detection probabilities for each of the five dependence levels for low probabilities of detection for the initial theft action, MC\&A observations, and the detection of moving materials. Some of these probabilities should be considered 


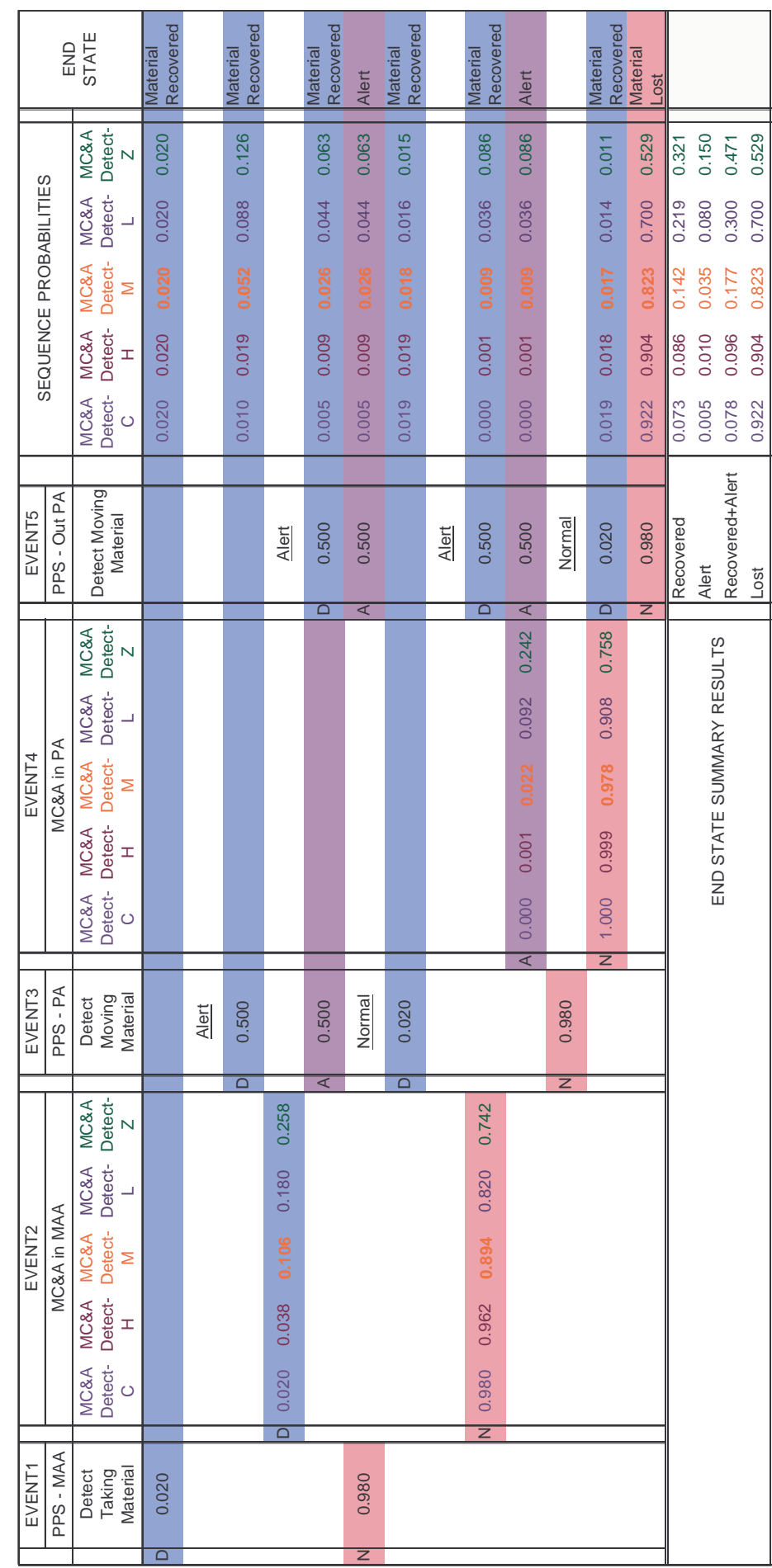

Figure 27: Event sequence calculations for the 30-day MAA/30-day PA timeline scenarios for 0.02 initial probability of MC\&A detection and five levels of dependence. 
artificially low because no real facility would be permitted to operate with such poor PPS and MC\&A performance. In this figure, the events shaded in blue are for detection and result in an end state of "Material Recovered," those in purple are for MC\&A "Alert" states, and those in pink are for "Material Lost." As expected from the dependence relationships, except for the first end state, the values for the individual sequence end states increase as dependence among MC\&A observations decreases from complete to zero dependence. This decrease factor varies from about 9 to about 12 .

The end state summary results are also provided for this scenario in Figure 27. The total probability for the "Material Recovered" end states increases from 0.073 to 0.321 (over 3 and a half times) as dependence among MC\&A observations moves from complete to zero dependence. The total probability for the "Alert" end states increases from 0.005 to 0.150 (almost 30 times). The probability that the facility knows the material is missing before it is taken offsite, which combines the "Material Recovered" and the "Alert" end states, increases from 0.078 to 0.471 (over 5 times) as independence among MC\&A observations is achieved.

It is also important to note how the consideration of MC\&A observations affects the analyst's perception of the likelihood of adversary success for an insider theft scenario. For this scenario, with no MC\&A detection, the total sequence probabilities for the "Material Recovered" and "Material Lost" would be 0.059 and 0.941 , respectively. Including in each physical protection layer one daily MC\&A activity with a low initial probability of detection improves the probability of recovering the material from over $20 \%$ for complete dependence to more than four times for zero dependence for MC\&A observations. Including $\mathrm{MC} \& \mathrm{~A}$ detection improves the probability that the facility knows material is missing before it is taken offsite from over $30 \%$ to almost seven times as dependence among MC\&A observations decreases from complete to zero dependence. 
Figures 28 and 29 show similar event sequence calculations for the medium (0.50) and high (0.99) initial probabilities of MC\&A detection, respectively. In each case, the same type of increases with decreasing dependence among MC\&A observations are evident for the individual event sequences as well as for the "Material Recovered" and "Alert" end state summaries. For the 0.50 initial MC\&A detection probability, the "Material Recovered," and "Alert" end states almost double (increase from 0.529 to 0.998). Including in each physical protection one daily MC\&A activity with a medium initial probability of detection improves the probability of recovering the material from about 8 times to about 16 times with decreasing dependence among MC\&A observations. For the 0.99 initial MC\&A detection probability, the total probability for the "Material Recovered" and "Alert" end states increases about 16 times over not including MC\&A detection.

\subsubsection{Variations of Timelines in the MAA and PA}

The previous section described the analysis of an adversary timeline in which the time delay between each of the discontinuous events was defined as a uniform distribution over 30 days. This section and the next two explore how the characteristics of the adversary timeline and the MC\&A detection timeline affect the security system effectiveness computed by this method.

The characteristics of the adversary timeline are affected by the scheduling of events that the adversary chooses to use or vulnerabilities he chooses to exploit in an attack scenario, as well as the adversary's knowledge of when the events occur. Both of these effects are captured in the probability distributions used to represent the adversary timelines. For this work, three types of timelines are used, each of which represent different conditions: 


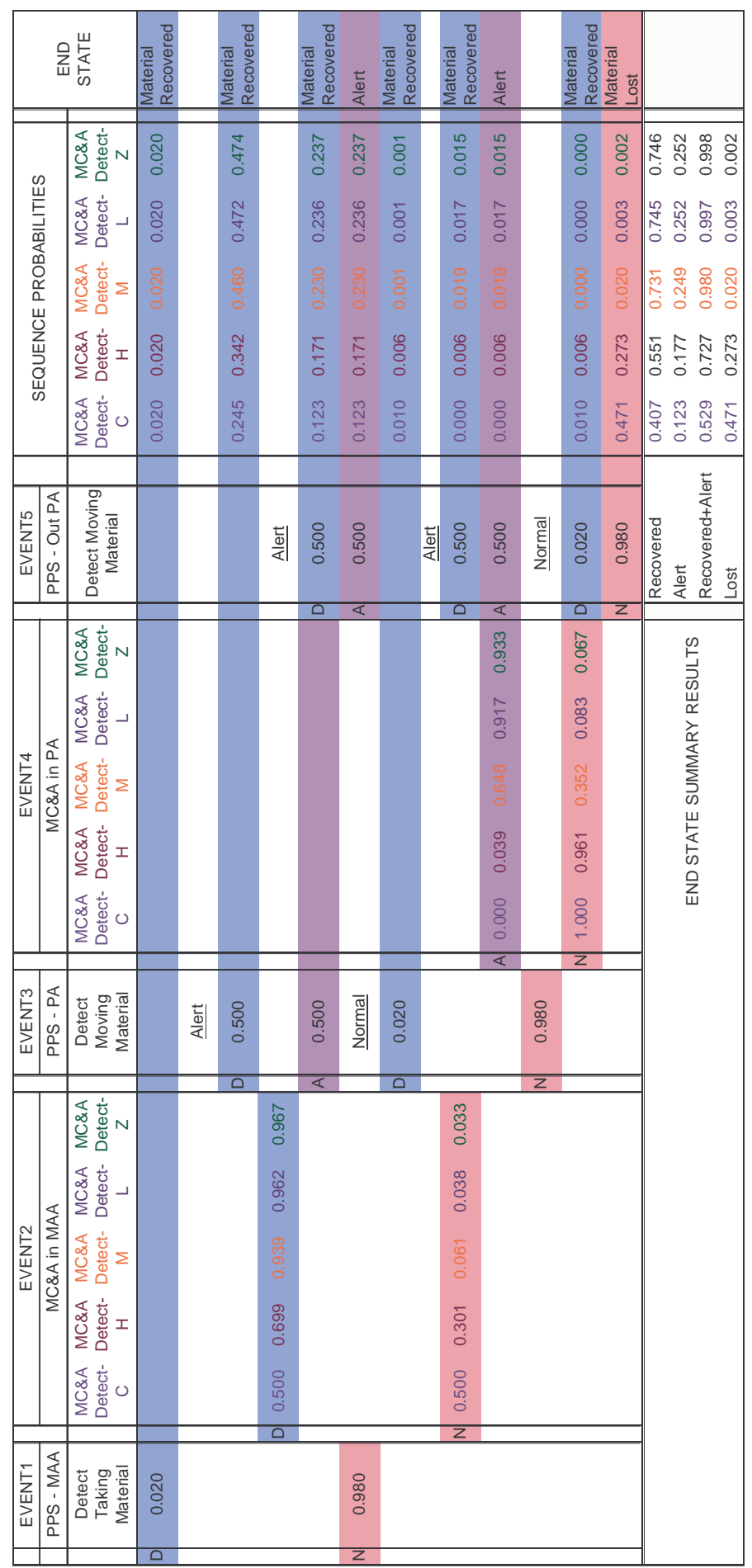

Figure 28: Event sequence calculations for the 30-day MAA/30-day PA timeline scenarios for 0.50 initial probability of MC\&A detection and five levels of dependence. 


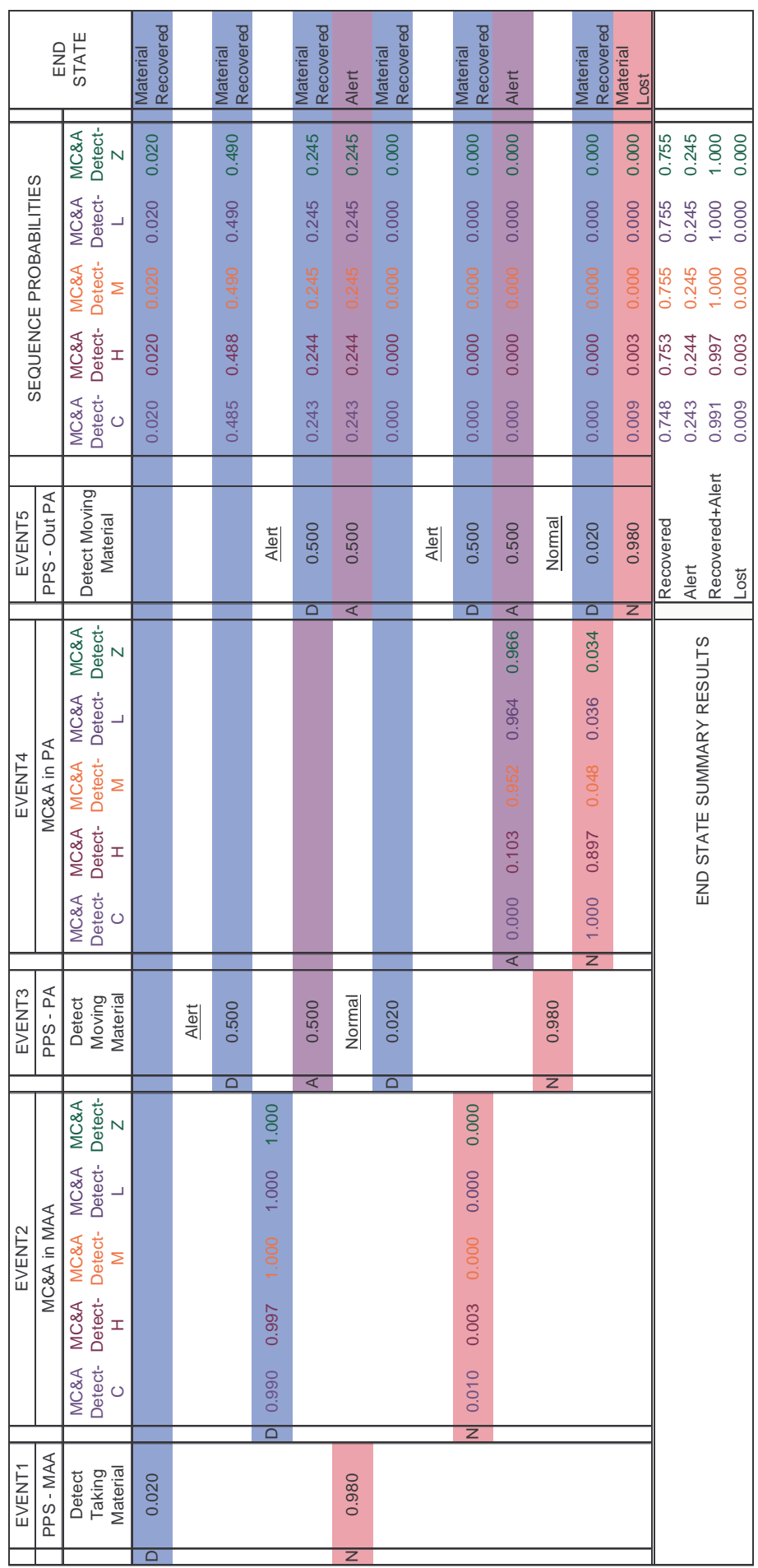

Figure 29: Event sequence calculations for the 30-day MAA/30-day PA timeline scenarios for 0.99 initial probability of MC\&A detection and five levels of dependence. 
- Uniform timeline - timeline for a condition that occurs at regular intervals; the interval is known to the insider, but the specific schedule is not; timeline is represented by a uniform probability distribution.

- Fixed timeline - timeline for a condition that occurs a fixed duration after a previous enabling condition; the enabling condition and fixed duration are known to the insider; the timeline is represented by a fixed delay time.

- Uncertain timeline - timeline for a condition that occurs randomly with a specific (estimated) likelihood each day; the timeline is represented by a geometric probability distribution.

The duration of the delay between discontinuous tasks is captured in the parameters of the distribution. Convolution must be used to calculate the values used in the model from the distributions. This section examines four additional adversary theft timelines that make use of different delays that are modeled as fixed durations and uniform durations in order to demonstrate how the MC\&A detection probabilities and overall event sequence probabilities vary for changes in the delays the adversary will encounter between discontinuous scenario events. The next two sections examines a more realistic facility analysis by using a set of activities to determine the MC\&A detection probabilities, first with a uniform adversary theft timeline (Section 4.3.3) and then with a geometric distribution for the adversary theft timeline (Section 4.3.4). Table 5 presents the four adversary timelines evaluated in this section. The example from Section 4.3.1 (Timeline 2) is included as a point of comparison.

Timely MC\&A detection for Event 2 is determined as described in Section 3.6.2. Table 6 presents the MC\&A detection probabilities for Event 2 for each of the five different timeline scenarios. Comparing the three timeline durations within a single dependence level, with the exception of complete dependence, the longer the timeline for 
Table 5: $\quad$ Five adversary timelines

\begin{tabular}{|c|c|c|c|c|}
\hline Timeline & Event 2 & Delay 2 & Event 4 & Delay 2 \\
\hline 1 & \multirow{5}{*}{ MC\&A in MAA } & $\begin{array}{l}5 \text { days - uniform } \\
\text { distribution }\end{array}$ & \multirow{5}{*}{ MC\&A in PA } & $\begin{array}{l}30 \text { days - uniform } \\
\text { distribution }\end{array}$ \\
\hline 2 & & $\begin{array}{l}30 \text { days - uniform } \\
\text { distribution }\end{array}$ & & $\begin{array}{l}30 \text { days - uniform } \\
\text { distribution }\end{array}$ \\
\hline 3 & & $\begin{array}{l}90 \text { days - uniform } \\
\text { distribution }\end{array}$ & & $\begin{array}{l}30 \text { days - uniform } \\
\text { distribution }\end{array}$ \\
\hline 4 & & $\begin{array}{c}5 \text { days - uniform } \\
\text { distribution }\end{array}$ & & 5 days - fixed delay \\
\hline 5 & & $\begin{array}{l}5 \text { days - uniform } \\
\text { distribution }\end{array}$ & & $\begin{array}{l}30 \text { days - fixed } \\
\text { delay }\end{array}$ \\
\hline
\end{tabular}

Table 6: Event 2 probability of timely MC\&A detection for the five scenario timelines for five dependence levels and low (0.02), medium (0.50), and high (0.99) initial probability of detection

\begin{tabular}{|l|c|c|c|c|c|c|}
\hline Timeline & Initial & \multicolumn{5}{|c|}{ Dependence } \\
\cline { 2 - 7 } MAA/PA & $\boldsymbol{P}_{\boldsymbol{D}, \boldsymbol{M C} \boldsymbol{A} \boldsymbol{A}}$ & Complete & High & Moderate & Low & Zero \\
\hline 1. 5-day Uniform/ & 0.02 & 0.020 & 0.032 & 0.049 & 0.055 & 0.058 \\
\cline { 2 - 7 } 30-day Uniform & 0.50 & 0.500 & 0.638 & 0.764 & 0.792 & 0.806 \\
\cline { 2 - 7 } & 0.99 & 0.990 & 0.995 & 0.998 & 0.998 & 0.998 \\
\hline \multirow{3}{*}{ 2. 30-day Uniform/ } & 0.02 & 0.020 & 0.038 & 0.106 & 0.180 & 0.258 \\
\cline { 2 - 7 } 30-day Uniform & 0.50 & 0.500 & 0.699 & 0.939 & 0.962 & 0.967 \\
\cline { 2 - 7 } & 0.99 & 0.990 & 0.997 & 1.000 & 1.000 & 1.000 \\
\hline \multirow{3}{*}{ 3. 90-day Uniform/ } & 0.02 & 0.020 & 0.039 & 0.123 & 0.269 & 0.544 \\
\cline { 2 - 7 } 30-day Uniform & 0.50 & 0.500 & 0.707 & 0.969 & 0.987 & 0.989 \\
\cline { 2 - 7 } & 0.99 & 0.990 & 0.997 & 0.999 & 0.999 & 0.999 \\
\hline 4. 5-day Uniform/ & 0.02 & 0.020 & 0.032 & 0.049 & 0.055 & 0.058 \\
\cline { 2 - 7 } 5-day Fixed & 0.50 & 0.500 & 0.638 & 0.764 & 0.792 & 0.806 \\
\cline { 2 - 7 } & 0.99 & 0.990 & 0.995 & 0.998 & 0.998 & 0.998 \\
\hline 5. 5-day Uniform/ & 0.02 & 0.020 & 0.032 & 0.049 & 0.055 & 0.058 \\
\cline { 2 - 7 } 30-day Fixed & 0.50 & 0.500 & 0.638 & 0.764 & 0.792 & 0.806 \\
\cline { 2 - 7 } & 0.99 & 0.990 & 0.995 & 0.998 & 0.998 & 0.998 \\
\hline
\end{tabular}

MC\&A detection in the MAA, the higher the probability of timely MC\&A detection. Similarly, across dependence levels, the probability of detection increases more with decreasing dependence between MC\&A observations for a longer timeline in the MAA. 
These results again emphasize the importance of extending an insider's theft timeline to increase the facility's probability of detecting suspicious or unauthorized actions.

The other significant difference among the timeline scenarios is the one-time theft opportunity in the PA for the last two timeline scenarios listed above. Having only one opportunity to move the material in the PA reduces significantly the calculations for the number of possible composite timelines, each of which has a higher individual probability (1/5 for the 5-day uniform/30-fixed timeline compared to $1 / 150$ for the 5-day uniform/30-day uniform timeline). With a fixed timeline in the PA, the total duration of the composite timelines will vary from six to ten days for the 5-day uniform/5-day fixed timeline and from 31 to 35 days for the 5-day uniform/30-day fixed timeline. For MC\&A detection in the PA, Table 7 presents the MC\&A detection probabilities for Event 4 in the ESD. Reducing the opportunity for moving material in the PA essentially removes theft scenarios of two to five days for the 5-day uniform/5-day fixed timeline and two to 30 days for the 5-day uniform/30-day fixed timeline. Thus, the shortest adversary scenarios timelines are prevented, so there is greater opportunity for MC\&A observations to detect the material as missing before it is removed from the facility. The resulting MC\&A detection probabilities in the PA generally increase compared to a uniform timeline in the PA - for example 0.086, 0.931, and 0.999 for the low, moderate and high initial probability of detection, for moderate dependence between MC\&A observations for the 5-day uniform/30-day fixed composite timeline compared to $0.067,0.831$, and 0.956 , respectively, for the 5-day uniform/30-day uniform composite timeline.

Another point to note for the Event $4 \mathrm{MC} \& \mathrm{~A}$ detection probabilities is the very low values for complete and high dependence. The low values are independent of distribution type and somewhat independent of duration and initial probability of MC\&A 
Table 7: $\quad$ Event 4 probability of timely MC\&A detection for the five scenario timelines for five dependence levels and low (0.02), medium (0.50), and high (0.99) initial probability of detection

\begin{tabular}{|l|c|c|c|c|c|c|}
\hline \multirow{2}{*}{ Timeline } & Initial & \multicolumn{5}{|c|}{ Dependence of MC\&A Detection Activities } \\
\cline { 2 - 7 } & $\boldsymbol{P}_{\boldsymbol{D}, \boldsymbol{M C} \boldsymbol{A}}$ & Complete & High & Moderate & Low & Zero \\
\hline \multirow{3}{*}{ 1. 5-day Uniform/ } & 0.02 & 0.000 & 0.007 & 0.067 & 0.149 & 0.242 \\
\cline { 2 - 7 } 30-day Uniform & 0.50 & 0.000 & 0.189 & 0.831 & 0.920 & 0.933 \\
\cline { 2 - 7 } & 0.99 & 0.000 & 0.397 & 0.956 & 0.964 & 0.966 \\
\hline 2. 30-day Uniform/ & 0.02 & 0.000 & 0.001 & 0.022 & 0.092 & 0.242 \\
\cline { 2 - 7 } 30-day Uniform & 0.50 & 0.000 & 0.039 & 0.648 & 0.917 & 0.933 \\
\cline { 2 - 7 } & 0.99 & 0.000 & 0.103 & 0.952 & 0.964 & 0.966 \\
\hline 3. 90-day Uniform/ & 0.02 & 0.000 & $4 \mathrm{E}-04$ & 0.007 & 0.042 & 0.242 \\
\cline { 2 - 7 } 30-day Uniform & 0.50 & 0.000 & 0.013 & 0.426 & 0.916 & 0.933 \\
\cline { 2 - 7 } & 0.99 & 0.000 & 0.037 & 0.944 & 0.964 & 0.966 \\
\hline 4. 5-day Uniform/ & 0.02 & 0.000 & 0.007 & 0.041 & 0.062 & 0.078 \\
\cline { 2 - 7 } 5-day Fixed & 0.50 & 0.000 & 0.192 & 0.745 & 0.886 & 0.938 \\
\cline { 2 - 7 } & 0.99 & 0.000 & 0.406 & 0.987 & 1.000 & 1.000 \\
\hline 5. 5-day Uniform/ & 0.02 & 0.000 & 0.008 & 0.086 & 0.235 & 0.443 \\
\cline { 2 - 7 } 30-day Fixed & 0.50 & 0.000 & 0.202 & 0.931 & 1.000 & 1.000 \\
\cline { 2 - 7 } & 0.99 & 0.000 & 0.420 & 0.999 & 1.000 & 1.000 \\
\hline
\end{tabular}

detection. These higher levels of dependence make later MC\&A observations less effective (in fact, MC\&A detection probability in the PA is 0 for complete dependence). If material has not been detected missing by the time it is moved out of the MAA, it is unlikely that it will be detected as missing while it is still in the PA.

Tables 8 through 12 present the end state summaries for the five different timelines. It is evident from these results that MC\&A detection as an alert provides an additional significant contribution to overall detection of an insider theft. Figure 30 is a plot of the results for the three uniform composite timelines that shows the general trends of increasing probability for the alert and material recovered end states with decreasing dependence between MC\&A observations and increasing timelines for the PA. Figure 31 is a plot of the results for the three 5-day MAA timelines with the respective uniform or fixed PA timelines. 
Table 8: $\quad$ End state summary results for Timeline 1 - 5-day MAA/30-day PA timeline

\begin{tabular}{|c|c|c|c|c|c|c|}
\hline \multirow{2}{*}{$\begin{array}{c}\text { Initial } \\
P_{D, M C \& A}\end{array}$} & \multirow{2}{*}{ End State } & \multicolumn{5}{|c|}{ Dependence of MC\&A Detection Activities } \\
\hline & & Complete & High & Moderate & Low & Zero \\
\hline \multirow{4}{*}{0.02} & Material Recovered & 0.073 & 0.084 & 0.122 & 0.162 & 0.205 \\
\hline & Alert & 0.005 & 0.011 & 0.043 & 0.081 & 0.124 \\
\hline & $\begin{array}{l}\text { Material Recovered } \\
+ \text { Alert }\end{array}$ & 0.078 & 0.096 & 0.165 & 0.243 & 0.329 \\
\hline & Material Lost & 0.922 & 0.904 & 0.835 & 0.757 & 0.671 \\
\hline \multirow{4}{*}{0.50} & Material Recovered & 0.407 & 0.535 & 0.681 & 0.699 & 0.703 \\
\hline & Alert & 0.123 & 0.189 & 0.281 & 0.286 & 0.284 \\
\hline & $\begin{array}{l}\text { Material Recovered } \\
+ \text { Alert }\end{array}$ & 0.529 & 0.724 & 0.963 & 0.984 & 0.988 \\
\hline & Material Lost & 0.471 & 0.276 & 0.037 & 0.016 & 0.012 \\
\hline \multirow{4}{*}{0.99} & Material Recovered & 0.748 & 0.752 & 0.754 & 0.754 & 0.754 \\
\hline & Alert & 0.243 & 0.245 & 0.246 & 0.246 & 0.246 \\
\hline & $\begin{array}{l}\text { Material Recovered } \\
+ \text { Alert }\end{array}$ & 0.991 & 0.997 & $\sim 0.999$ & $\sim 1.000$ & $\sim 1.000$ \\
\hline & Material Lost & 0.009 & 0.003 & 1E-04 & $7 E-05$ & $6 \mathrm{E}-05$ \\
\hline
\end{tabular}

These results further reinforce the insights from the analysis in the previous section, namely:

- Decreasing dependence among MC\&A observations increase the sequence probabilities for the Material Recovered and Alert end states. MC\&A activities with at most a moderate level of dependence between observations can provide significant improvement in overall effectiveness.

- Longer timelines improve detection effectiveness. Forcing the adversary to keep material in a physical protection layer longer provides more opportunity for detection so that even low initial probabilities of MC\&A detection can result in a significantly higher cumulative probability of detection.

- Higher initial probabilities of MC\&A detection for an activity can accommodate a higher level of dependence between MC\&A observations, although less opportunity is available to improve overall cumulative probability of detection. 
Table 9: $\quad$ End state summary results for Timeline 2 - 30-day MAA/30-day PA timeline

\begin{tabular}{|c|c|c|c|c|c|c|}
\hline \multirow{2}{*}{$\begin{array}{c}\text { Initial } \\
P_{D, M C \& A}\end{array}$} & \multirow{2}{*}{ End State } & \multicolumn{5}{|c|}{ Dependence of MC\&A Detection Activities } \\
\hline & & Complete & High & Moderate & Low & Zero \\
\hline \multirow{4}{*}{0.02} & Material Recovered & 0.073 & 0.086 & 0.142 & 0.219 & 0.321 \\
\hline & Alert & 0.005 & 0.010 & 0.035 & 0.080 & 0.150 \\
\hline & $\begin{array}{l}\text { Material Recovered } \\
+ \text { Alert }\end{array}$ & 0.078 & 0.096 & 0.177 & 0.300 & 0.471 \\
\hline & Material Lost & 0.922 & 0.904 & 0.823 & 0.700 & 0.529 \\
\hline \multirow{4}{*}{0.50} & Material Recovered & 0.407 & 0.551 & 0.731 & 0.745 & 0.746 \\
\hline & Alert & 0.123 & 0.177 & 0.249 & 0.252 & 0.252 \\
\hline & $\begin{array}{l}\text { Material Recovered } \\
+ \text { Alert }\end{array}$ & 0.529 & 0.727 & 0.980 & 0.997 & 0.998 \\
\hline & Material Lost & 0.471 & 0.273 & 0.020 & 0.003 & 0.002 \\
\hline \multirow{4}{*}{0.99} & Material Recovered & 0.748 & 0.753 & 0.755 & 0.755 & 0.755 \\
\hline & Alert & 0.243 & 0.244 & 0.245 & 0.245 & 0.245 \\
\hline & $\begin{array}{l}\text { Material Recovered } \\
+ \text { Alert }\end{array}$ & 0.991 & 0.997 & $\sim 1.000$ & $\sim 1.000$ & $\sim 1.000$ \\
\hline & Material Lost & 0.009 & 0.003 & $2 \mathrm{E}-05$ & $1 \mathrm{E}-05$ & $1 \mathrm{E}-05$ \\
\hline
\end{tabular}

Table 10: $\quad$ End state summary results for Timeline 3 - 90-day MAA/30-day PA timeline

\begin{tabular}{|c|c|c|c|c|c|c|}
\hline \multirow{2}{*}{$\begin{array}{l}\text { Initial } \\
\boldsymbol{P}_{D, M C \& A}\end{array}$} & \multirow{2}{*}{ End State } & \multicolumn{5}{|c|}{ Dependence of MC\&A Detection Activities } \\
\hline & & Complete & High & Moderate & Low & Zero \\
\hline \multirow{4}{*}{0.02} & Material Recovered & 0.073 & 0.086 & 0.147 & 0.260 & 0.488 \\
\hline & Alert & 0.005 & 0.010 & 0.033 & 0.081 & 0.186 \\
\hline & $\begin{array}{l}\text { Material Recovered } \\
+ \text { Alert }\end{array}$ & 0.078 & 0.096 & 0.180 & 0.341 & 0.674 \\
\hline & Material Lost & 0.922 & 0.904 & 0.820 & 0.659 & 0.326 \\
\hline \multirow{4}{*}{0.50} & Material Recovered & 0.407 & 0.553 & 0.739 & 0.752 & 0.752 \\
\hline & Alert & 0.123 & 0.175 & 0.244 & 0.247 & 0.247 \\
\hline & $\begin{array}{l}\text { Material Recovered } \\
+ \text { Alert }\end{array}$ & 0.530 & 0.728 & 0.983 & 0.999 & 0.999 \\
\hline & Material Lost & 0.470 & 0.272 & 0.017 & 0.001 & 0.001 \\
\hline \multirow{4}{*}{0.99} & Material Recovered & 0.748 & 0.753 & 0.755 & 0.755 & 0.755 \\
\hline & Alert & 0.243 & 0.244 & 0.245 & 0.245 & 0.245 \\
\hline & $\begin{array}{l}\text { Material Recovered } \\
+ \text { Alert }\end{array}$ & 0.991 & 0.997 & $\sim 1.000$ & $\sim 1.000$ & $\sim 1.000$ \\
\hline & Material Lost & 0.009 & 0.003 & 7E-06 & 4E-06 & 4E-06 \\
\hline
\end{tabular}


Table 11: End state summary results for Timeline 4 - 5-day MAA/5-day fixed PA timeline

\begin{tabular}{|c|c|c|c|c|c|c|}
\hline \multirow{2}{*}{$\begin{array}{l}\text { Initial } \\
P_{D, M C \& A}\end{array}$} & \multirow{2}{*}{ End State } & \multicolumn{5}{|c|}{ Dependence of MC\&A Detection Activities } \\
\hline & & Complete & High & Moderate & Low & Zero \\
\hline \multirow{4}{*}{0.02} & Material Recovered & 0.073 & 0.084 & 0.111 & 0.124 & 0.133 \\
\hline & Alert & 0.005 & 0.011 & 0.031 & 0.042 & 0.049 \\
\hline & $\begin{array}{l}\text { Material Recovered } \\
+ \text { Alert }\end{array}$ & 0.078 & 0.096 & 0.142 & 0.166 & 0.182 \\
\hline & Material Lost & 0.922 & 0.904 & 0.858 & 0.834 & 0.818 \\
\hline \multirow{4}{*}{0.50} & Material Recovered & 0.407 & 0.535 & 0.672 & 0.695 & 0.704 \\
\hline & Alert & 0.123 & 0.190 & 0.272 & 0.282 & 0.285 \\
\hline & $\begin{array}{l}\text { Material Recovered } \\
+ \text { Alert }\end{array}$ & 0.530 & 0.725 & 0.944 & 0.977 & 0.989 \\
\hline & Material Lost & 0.470 & 0.275 & 0.056 & 0.023 & 0.011 \\
\hline \multirow{4}{*}{0.99} & Material Recovered & 0.748 & 0.752 & 0.754 & 0.754 & 0.755 \\
\hline & Alert & 0.243 & 0.245 & 0.246 & 0.246 & 0.245 \\
\hline & $\begin{array}{l}\text { Material Recovered } \\
+ \text { Alert }\end{array}$ & 0.991 & 0.997 & $\sim 1.000$ & $\sim 1.000$ & $\sim 1.000$ \\
\hline & Material Lost & 0.009 & 0.003 & $3 \mathrm{E}-05$ & 5E-07 & $2 \mathrm{E}-11$ \\
\hline
\end{tabular}

Table 12: End state summary results for Timeline 5 - 5-day MAA/30-day fixed PA timeline

\begin{tabular}{|c|c|c|c|c|c|c|}
\hline \multirow{2}{*}{$\begin{array}{l}\text { Initial } \\
P_{D, M C \& A}\end{array}$} & \multirow{2}{*}{ End State } & \multicolumn{5}{|c|}{ Dependence of MC\&A Detection Activities } \\
\hline & & Complete & High & Moderate & Low & Zero \\
\hline \multirow{4}{*}{0.02} & Material Recovered & 0.073 & 0.085 & 0.130 & 0.199 & 0.292 \\
\hline & Alert & 0.005 & 0.011 & 0.051 & 0.120 & 0.215 \\
\hline & $\begin{array}{l}\text { Material Recovered } \\
+ \text { Alert }\end{array}$ & 0.078 & 0.096 & 0.181 & 0.319 & 0.507 \\
\hline & Material Lost & 0.922 & 0.904 & 0.819 & 0.681 & 0.493 \\
\hline \multirow{4}{*}{0.50} & Material Recovered & 0.407 & 0.537 & 0.692 & 0.706 & 0.709 \\
\hline & Alert & 0.123 & 0.191 & 0.293 & 0.294 & 0.291 \\
\hline & $\begin{array}{l}\text { Material Recovered } \\
+ \text { Alert }\end{array}$ & 0.530 & 0.728 & 0.985 & $\sim 1.000$ & $\sim 1.000$ \\
\hline & Material Lost & 0.471 & 0.272 & 0.015 & $6 \mathrm{E}-05$ & $3 \mathrm{E}-10$ \\
\hline \multirow{4}{*}{0.99} & Material Recovered & 0.757 & 0.755 & 0.754 & 0.754 & 0.755 \\
\hline & Alert & 0.243 & 0.245 & 0.246 & 0.246 & 0.245 \\
\hline & $\begin{array}{l}\text { Material Recovered } \\
+ \text { Alert }\end{array}$ & 0.990 & $\sim 1.000$ & $\sim 1.000$ & $\sim 1.000$ & 1.000 \\
\hline & Material Lost & 0.009 & 0.003 & $2 \mathrm{E}-06$ & $1 \mathrm{E}-13$ & 0.000 \\
\hline
\end{tabular}




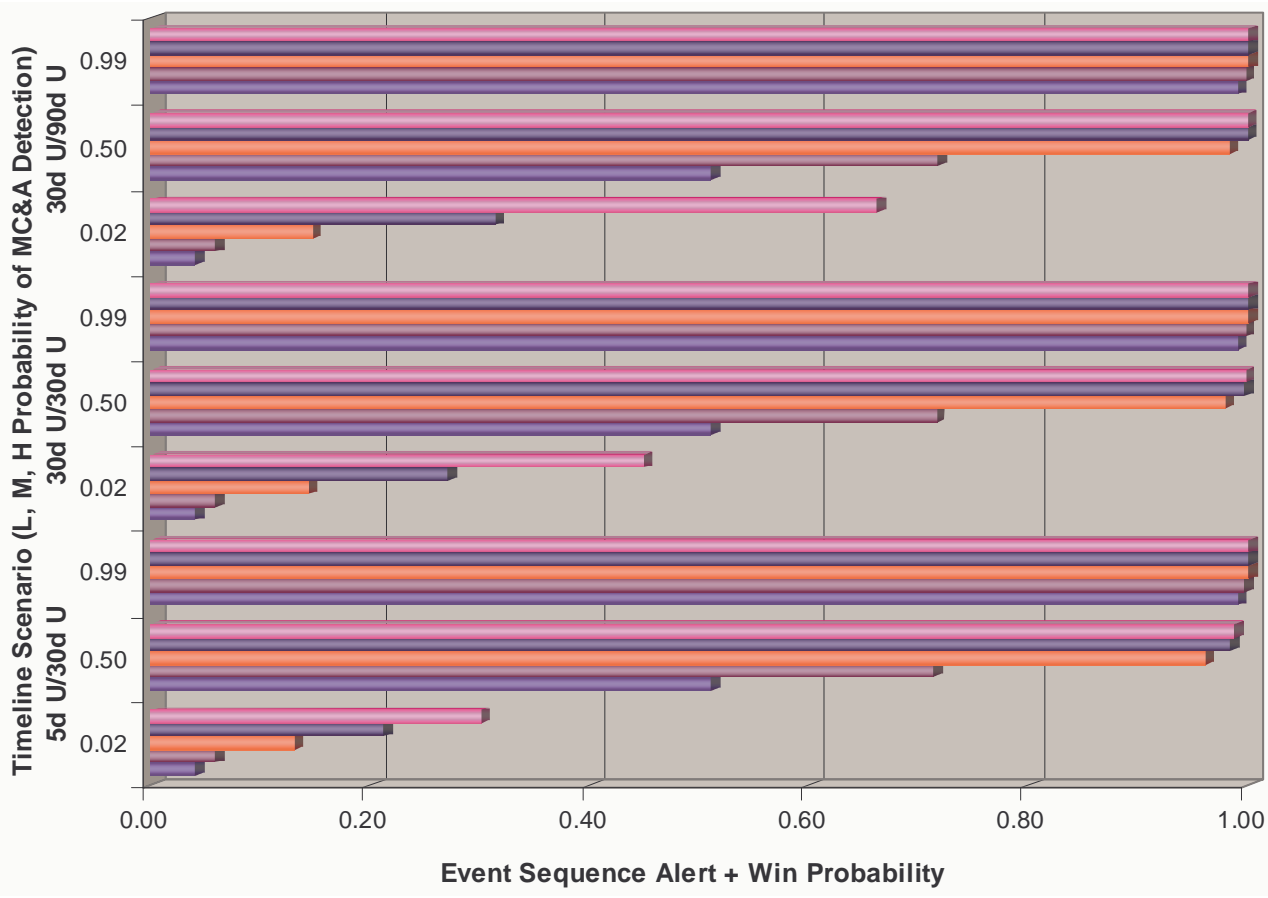

Figure 30: Plot of material recovered + alert end state summary results for the three uniform composite timelines

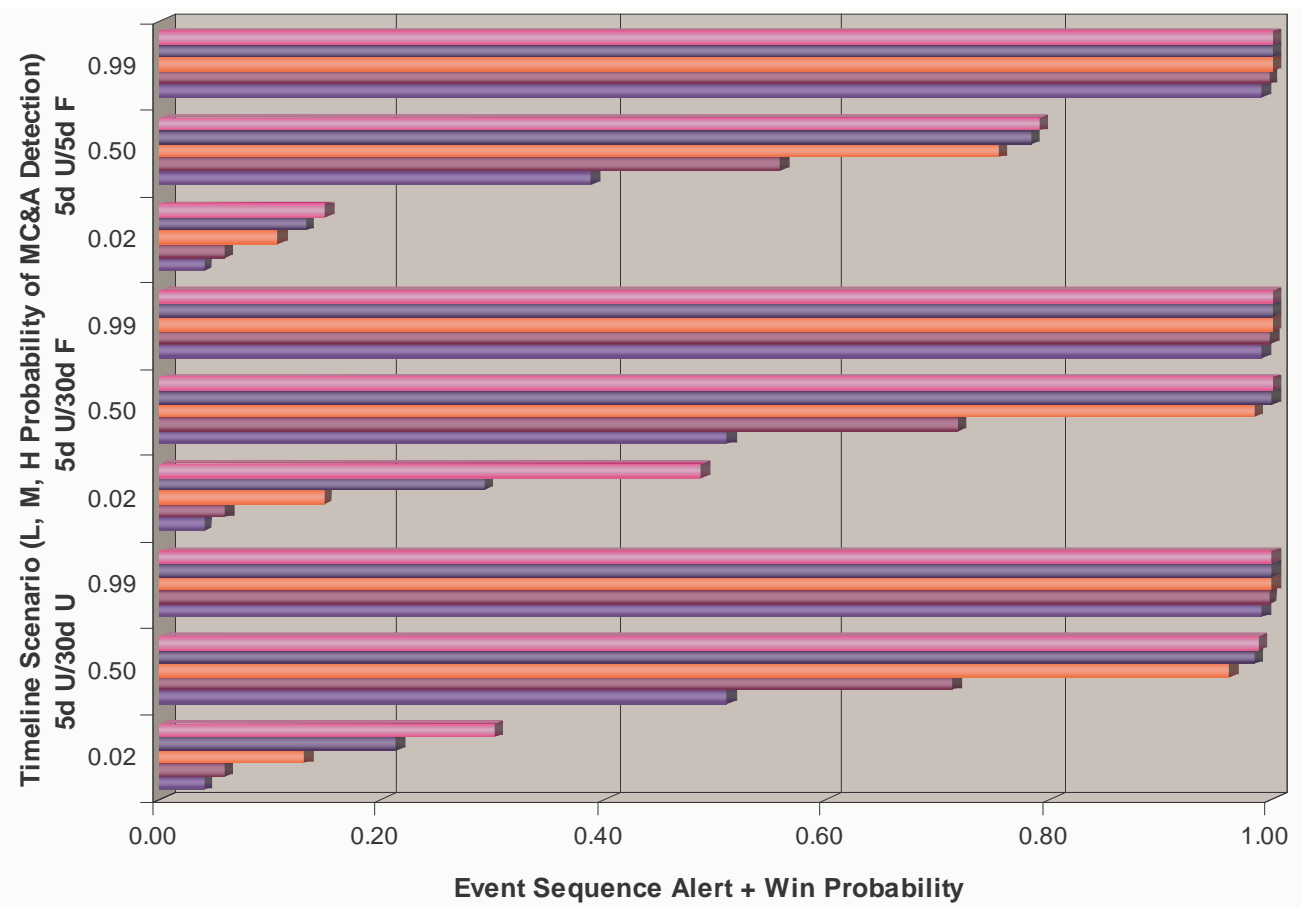

Figure 31: Plot of material recovered + alert end state summary results for the three 5-day MAA timelines and respective uniform and fixed PA timelines 


\subsubsection{Analysis for Facility-level MC\&A Operations}

To explore the proposed methods, the previous analyses demonstrate the extended path analysis methodology for the following limited conditions:

- One daily MC\&A activity

- Low, medium and high initial probabilities of MC\&A detection (complements of BHEPs associated with certain types of NPP operations)

- Detection timelines based on the dependency relationships between MC\&A observations

- Uniform and fixed timelines of varying durations and

- Multiple physical protection layers.

Actual facility-level MC\&A operations are much more complex and involve many MC\&A activities that are performed at various intervals. To demonstrate the extended path analysis methodology for scenarios that are more representative of the complexity of actual facility MC\&A operations, additional analyses were done for a 5-day MAA/30-day PA scenario timeline for a set of MC\&A activities that occur at different intervals.

Table 13 presents a detection opportunity timeline for a notional set of six MC\&A activities at a facility. Each of the six activities occurs at a different interval and has been assigned a BHEP as determined in Table 1. Also, each activity has been assigned a given level of dependence, and the day-to-day calculations of the BHEP reflect this dependence relationship. For example, the Forms Reconciliation activity, which occurs every three days, has a high level of dependence between each performance of this activity. The Process Call, which occurs every 14 days, has a moderate level of dependence between 


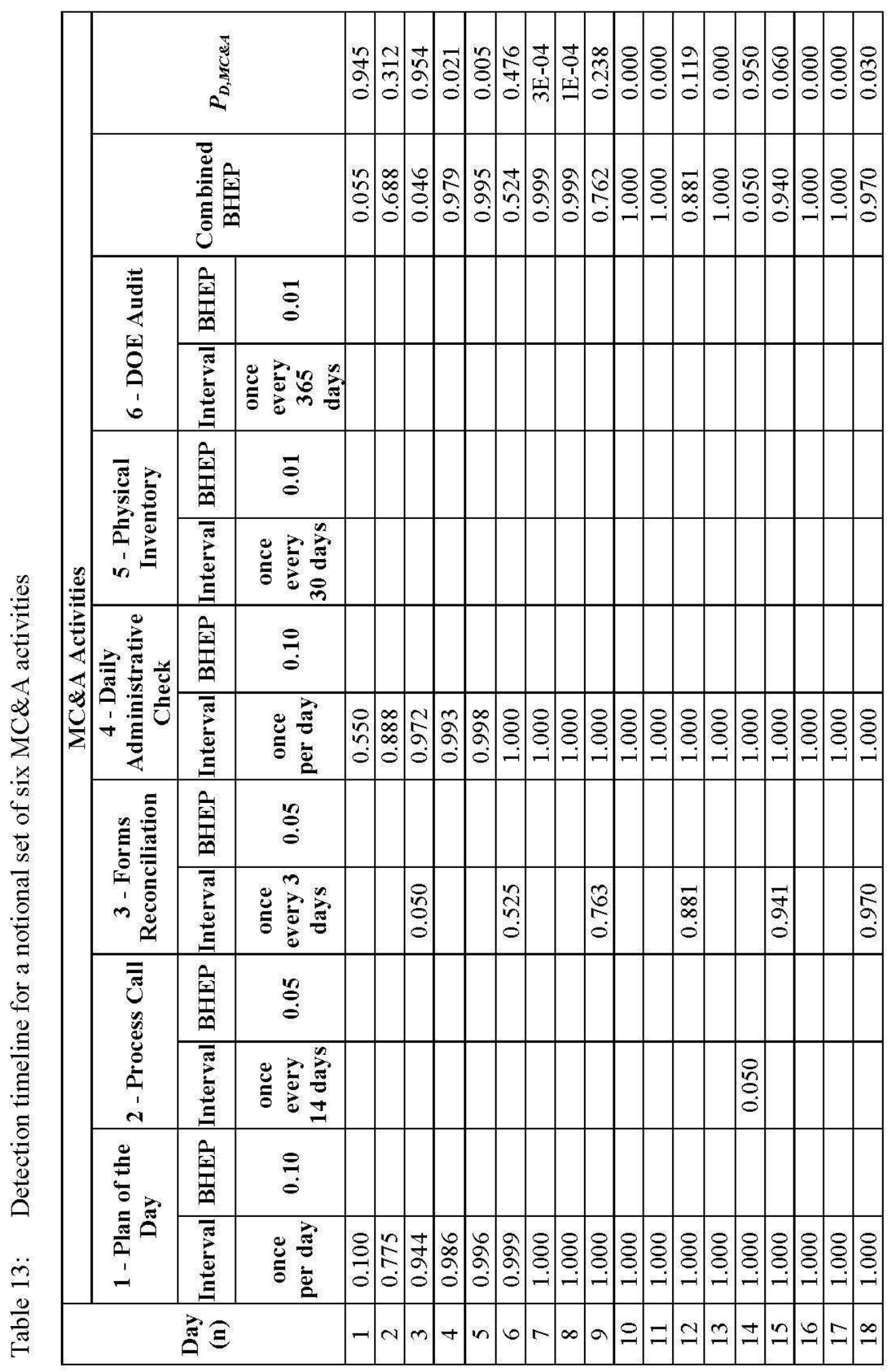




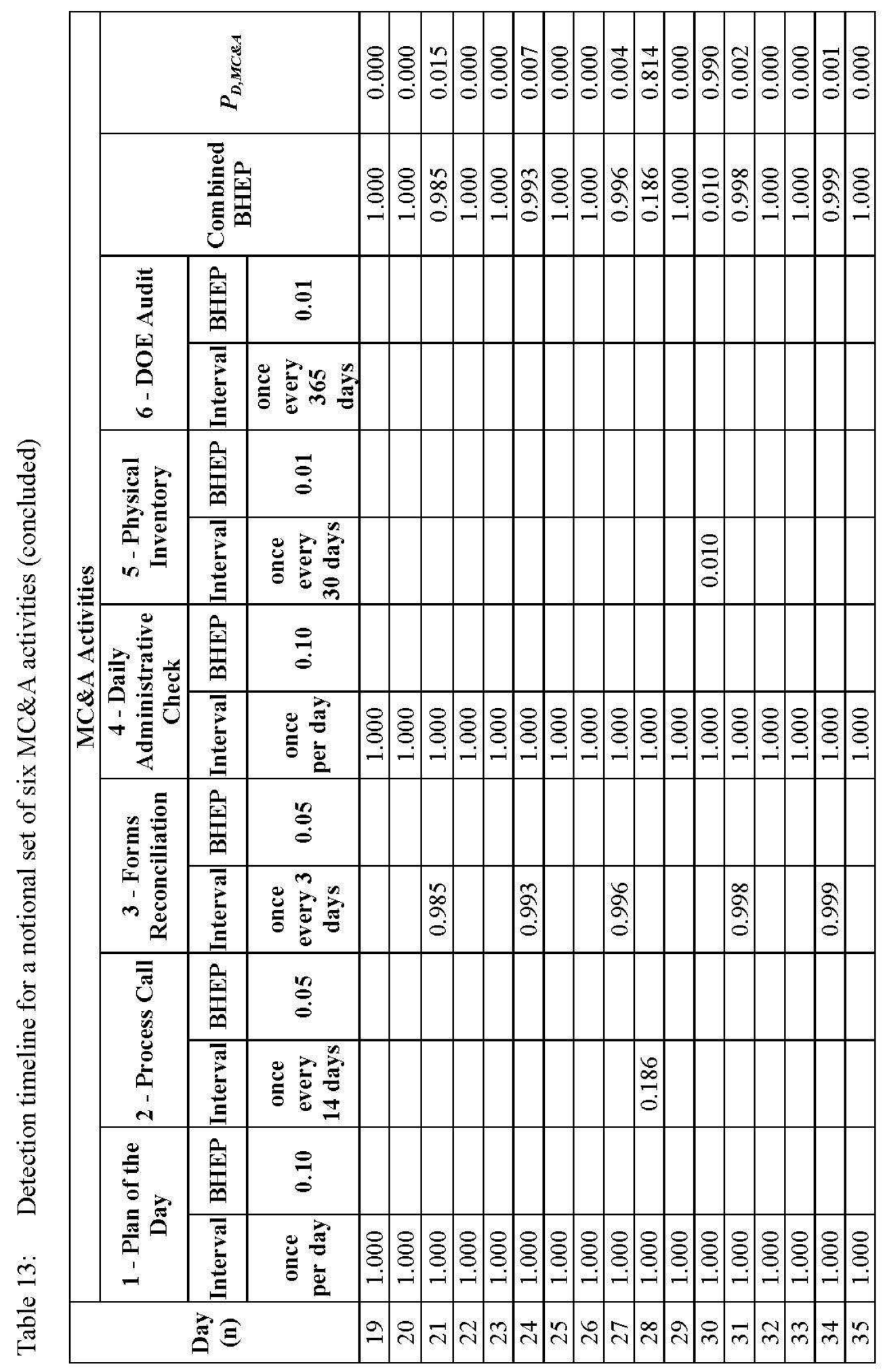


each performance of this activity. In this example, the Plan of the Day and Daily Administrative Check are performed once a day by the same person, so these activities are assigned a high level of dependence between the performance of each of these activities.

The daily probability of detection can be determined by combining the BHEPs as non-detection probabilities and taking the complement:

$$
P_{\text {Dayn }}=1-\prod_{m=1}^{M} B H E P_{m}
$$

For example, on Day 3, the set of MC\&A activities includes:

- 1 - Plan of the Day,

- 3 - Forms Reconciliation, and

- 4 - Daily Administrative Check.

and the daily probability of detection, $P_{M C \& A, 3}$, is calculated as:

$$
\begin{aligned}
P_{M C \& A, n} & =1-\prod_{m=1}^{M} B H E P_{m} \\
P_{M C \& A, 3} & =1-\left[\left(B H E P_{1}\right)\left(B H E P_{3}\right)\left(B H E P_{4}\right)\right] \\
P_{M C \& A, 3} & =1-[(0.944)(0.050)(0.972)] \\
P_{M C \& A, 3} & =1-[0.046] \\
P_{M C \& A, 3} & =0.954
\end{aligned}
$$

The probability of MC\&A detection on day three is higher than that for the previous two days because additional MC\&A activities have occurred on this day to contribute to a higher level of detection for the set of MC\&A activities. The MC\&A detection timeline for the scenario is determined from the daily probabilities of MC\&A detection. This detection timeline for 35 days is illustrated in Figure 32. Over the course of the 35-day timeline, the daily probability of MC\&A detection increases as additional activities occur to contribute to detection, or decreases as the dependence relationships reduce detection 
between observations. The underlying effect of the dependency relationships is also evident in Figure 32.

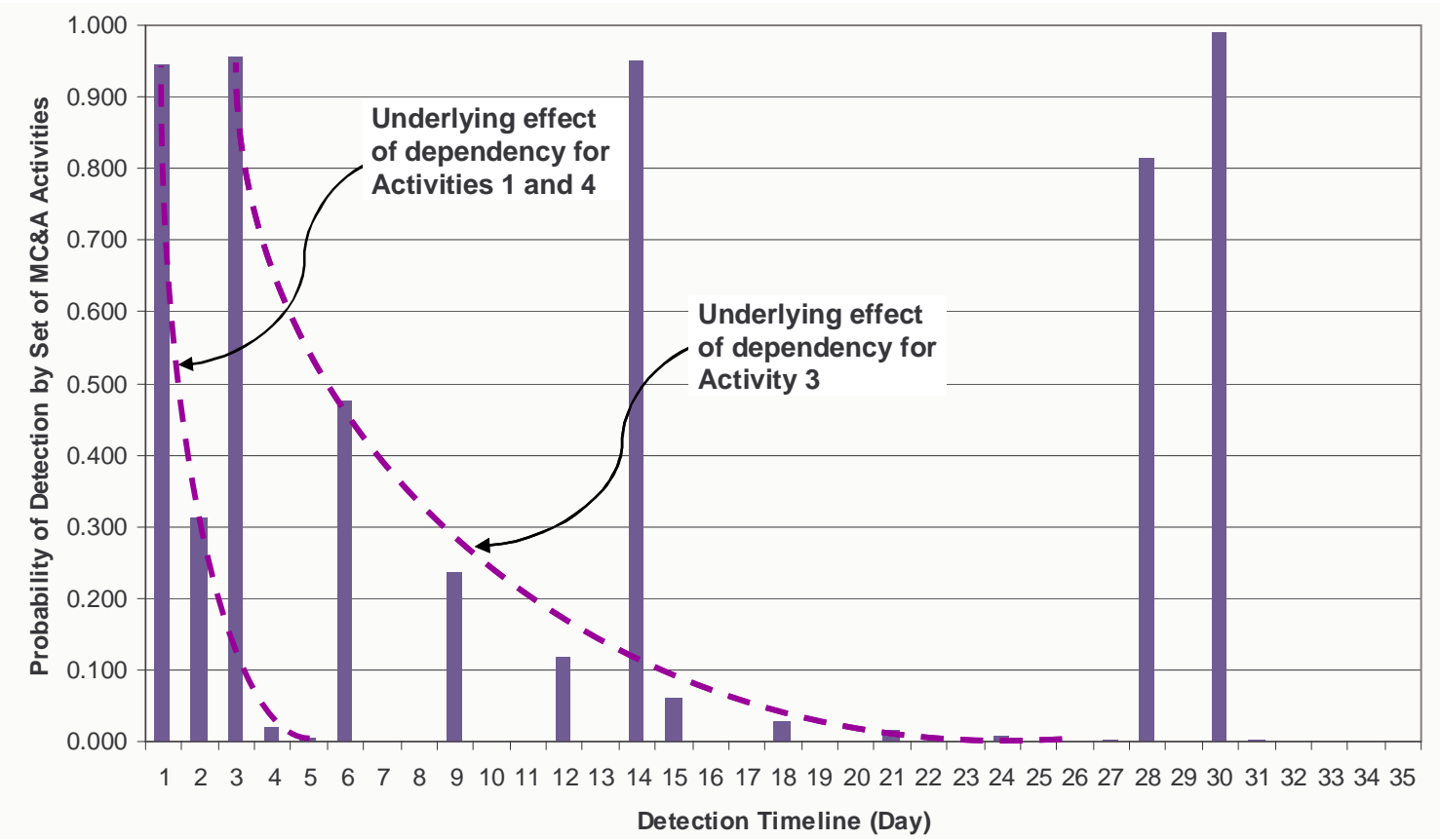

Figure 32: Daily probability of detection over a 35-day period for a set of MC\&A activities

The detection timeline for the set of MC\&A activities was evaluated against an adversary timeline in which Delay 1 for MC\&A in the MAA was represented as a 5-day uniform distribution and Delay 2 for MC\&A in the PA was represented as a 30-day uniform distribution. For the 5-day MAA timeline, the daily values of MC\&A detection for the first five days (Table 14) are used in the convolution calculation. For this case, timely MC\&A detection for Event 2 in the ESD is calculated to be 0.98 . For the composite MAA/PA timelines, the daily values of MC\&A detection for the 35-day composite timeline are used in the convolution calculation, and timely MC\&A detection for Event 4 in the ESD is calculated to be 0.938. The sequence probabilities for the Material Recovered and Alert end states are 0.750 and 0.249 , respectively. Thus, the set 
of MC\&A activities result in a level of MC\&A detection similar to that for a single MC\&A activity with a high initial probability of detection, even though some of the MC\&A activities in the set have high and moderate levels of dependence between observations and across activities.

This analysis demonstrates the applicability of the extended path analysis methods for more realistic facility conditions. The daily probability of detection in Figure 32 provides insights for evaluating the protection level provided by MC\&A activities over time and identifying gaps in that protection level. For example, daily probability of detection from days 15 through 27 indicate that additional protection is needed and action should be taken to reduce dependency in the performance of MC\&A activities, or to add other activities that would increase the protection level during that time period. The importance of MC\&A activities is also evident - while a single MC\&A activity has the potential to contribute significantly to cumulative detection, a set of activities has the potential to maintain cumulative detection over time.

\subsubsection{Addressing Uncertainty in Insider Theft Timelines}

To further address the complexity of actual insider theft scenarios, the detection timeline for the set of MC\&A activities described in Section 4.3.3 was used with an insider theft timeline composed of geometric distributions. The convolution of these distributions was computed using LHS sampling. This approach to determine an insider theft timeline reflects the uncertainty in an insider's theft timeline as well as an analyst's lack of knowledge about possible insider theft timelines.

In LHS, the convolution for the composite MAA/PA timeline was determined by sampling 2000 observations for two each of the distributions (MAATHEFT and PATHEFT) for a geometric distribution with three different values for probability of failure. The geometric distribution was selected because it represents the number of 
successful trials that might be observed before a failure occurs. For insider theft, the probability of failure is the probability that the facility will be in a vulnerable state that the malicious insider will find favorable enough to attempt to move material to the next physical protection layer. Figure 33 is a plot of each of the three geometric distributions over their first 30 days. In each composite theft timeline, the distributions for the MAA and PA theft timelines are the same. Thus the three composite timelines considered in this analysis are each composed of two identical, but uncorrelated geometric distributions with failure probabilities of $0.20,0.50$, and 0.80 , respectively.

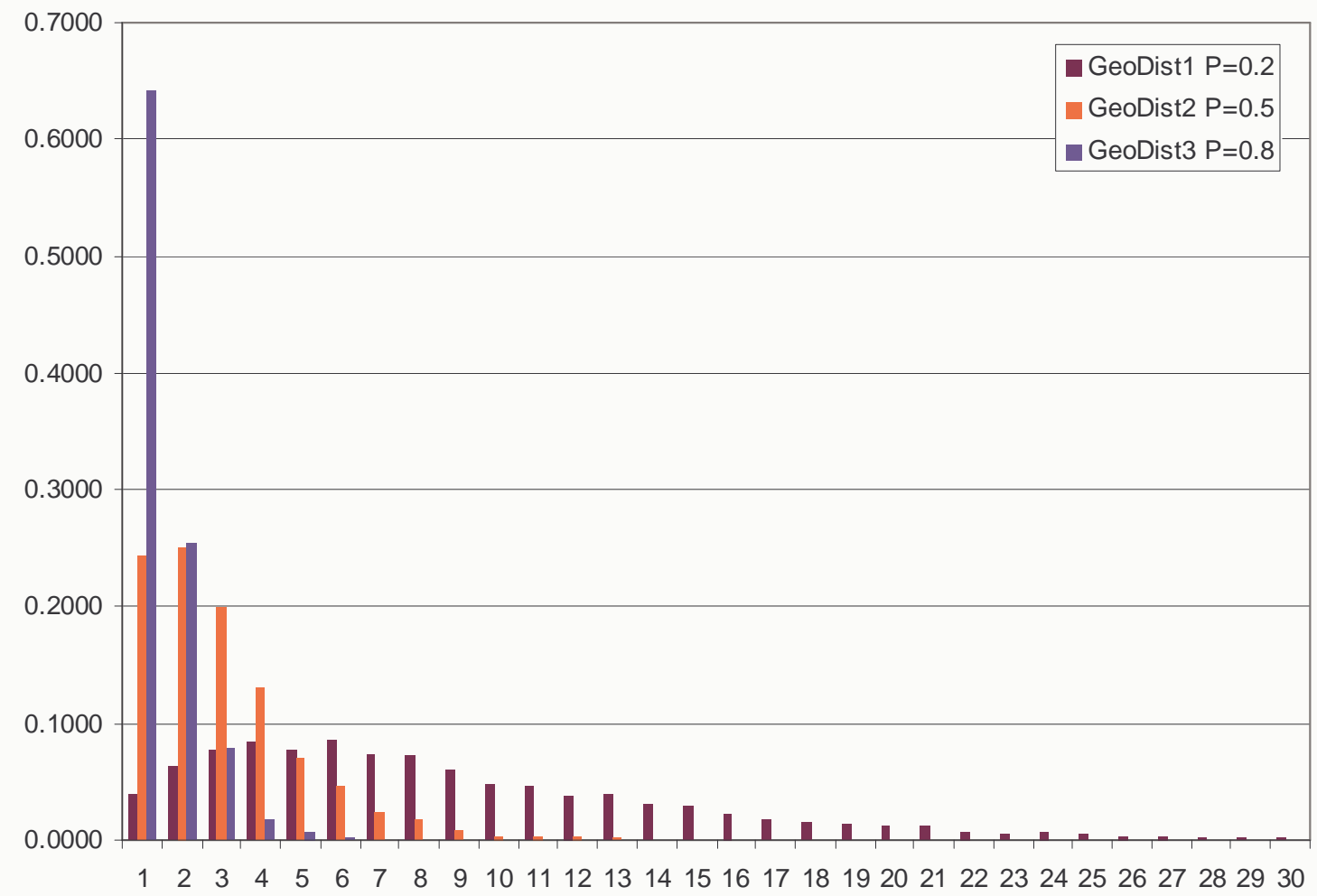

Figure 33: Geometric distributions for theft timeline generated from LHS.

To perform the calculations for timely MC\&A detection in the MAA, each geometric distribution was used in the calculations as described in Section 3.6.2. The 
calculations for timely detection in the PA require convolution of the distributions for the MAA theft timeline and the PA theft timeline, which was done as follows. LHS was used to draw 2000 observations for each distribution, and the values for each distribution were summed on an observation-by-observation basis to obtain observations for the total duration of the theft timeline. The probability of each unique theft timeline value was determined through a frequency analysis of the resulting observation set. The resulting set of probabilities was used to represent the theft timeline in the calculation of timely MC\&A detection in the PA with an MC\&A detection timeline for a set of MC\&A activities as describe in Section 4.3.3. Table 14 provides the values for timely MC\&A detection in the MAA and PA. Table 15 provides the end state summary results from the ESD calculations for the three geometric timeline scenarios.

Table 14: Timely MC\&A detection in the MAA (Event 2) and the PA (Event 4) for a set of MC\&A activities and geometric distributions for theft timeline

\begin{tabular}{|l|c|c|}
\hline \multirow{2}{*}{ Composite MAA/PA Timeline } & \multicolumn{2}{|c|}{ Timely MC\&A Detection } \\
\cline { 2 - 3 } & MAA - Event 2 & PA - Event 4 \\
\hline $\begin{array}{l}\text { Geometric Distribution 1 } \\
\mathrm{P}=0.20\end{array}$ & 0.629 & 0.702 \\
\hline $\begin{array}{l}\text { Geometric Distribution 2 } \\
\mathrm{P}=0.50\end{array}$ & 0.241 & 0.328 \\
\hline $\begin{array}{l}\text { Geometric Distribution 3 } \\
\mathrm{P}=0.80\end{array}$ & 0.038 & 0.064 \\
\hline
\end{tabular}

\subsubsection{Mitigating Potential Malicious Insider Activity}

The application of HRA methods has provided a probabilistic basis for incorporating MC\&A activities in an extended path analysis methodology. One purpose for analyzing a PPS is to identify vulnerabilities or gain insights on the possible impacts of additional protection elements. The final application of HRA methods for characterizing MC\&A activities was an exercise to demonstrate how these methods might be used to explore strategies for mitigating malicious insider activity. This 
Table 15: Comparison of end state summary results for 5-day MAA/30-day PA timeline and geometric distributions for theft timeline for a set of MC\&A activities

\begin{tabular}{|c|c|c|c|}
\hline Composite MAA/PA Timeline & $\begin{array}{l}\text { Initial } \\
\boldsymbol{P}_{D, M C \& A}\end{array}$ & End State & $\begin{array}{c}\text { Sequence } \\
\text { Probability }\end{array}$ \\
\hline \multirow{4}{*}{$\begin{array}{l}\text { Geometric Distribution } 1 \\
\mathrm{P}=0.20 \text { (mean time before material is } \\
\text { removed }-4 \text { days) }\end{array}$} & \multirow{4}{*}{0.945} & Material Recovered & 0.617 \\
\hline & & Alert & 0.279 \\
\hline & & $\begin{array}{l}\text { Material Recovered } \\
+ \text { Alert }\end{array}$ & 0.996 \\
\hline & & Material Lost & 0.004 \\
\hline \multirow{4}{*}{$\begin{array}{l}\text { Geometric Distribution } 2 \\
\mathrm{P}=0.50 \text { (mean time before material is } \\
\text { removed }-1 \text { day) }\end{array}$} & \multirow{4}{*}{0.945} & Material Recovered & 0.341 \\
\hline & & Alert & 0.179 \\
\hline & & $\begin{array}{l}\text { Material Recovered } \\
+ \text { Alert }\end{array}$ & 0.520 \\
\hline & & Material Lost & 0.480 \\
\hline \multirow{4}{*}{$\begin{array}{l}\text { Geometric Distribution } 3 \\
\mathrm{P}=0.80 \text { (mean time before material is } \\
\text { removed }-<1 \text { day) }\end{array}$} & \multirow{4}{*}{0.945} & Material Recovered & 0.114 \\
\hline & & Alert & 0.039 \\
\hline & & $\begin{array}{l}\text { Material Recovered } \\
+ \text { Alert }\end{array}$ & 0.153 \\
\hline & & Material Lost & 0.847 \\
\hline \multirow{4}{*}{ 5-day Uniform/30-day Uniform } & \multirow{4}{*}{0.945} & Material Recovered & 0.750 \\
\hline & & Alert & 0.249 \\
\hline & & $\begin{array}{l}\text { Material Recovered } \\
+ \text { Alert }\end{array}$ & 0.999 \\
\hline & & Material Lost & 0.001 \\
\hline
\end{tabular}

analysis used the 5-day MAA/5-day PA scenario timeline with uniform distributions for the theft timelines and the detection timeline developed for a set of MC\&A activities. This scenario timeline has a two-day to ten-day possible duration and 25 possible composite timelines. Three cases for the MC\&A detection timeline were addressed: one for the baseline set of combined MC\&A activities described in Table 13; a second assuming a malicious insider performs activities 1 and 4, which have a high level of dependence; and a third assuming the dependency relationship is removed for activity 4. The baseline case assumes that the insider has access to the material, but is not in a position of performing MC\&A tasks.

For the first ten-day composite timeline, the detection timeline used the daily MC\&A detection probabilities for the first ten days from the baseline set of combined 
MC\&A activities (Table 13). In this baseline set of activities, it was assumed that activities 1 and 4 are performed by the same person on a daily basis, and therefore they are assigned a high level of dependence between recurrences of these activities. The next variation for this timeline assumes that the person who performs activities 1 and 4 is a malicious insider who is seeking to steal material. Consequently, the BHEP for these activities is set to 1 and the probability of detection is 0 because the thief is concealing the activities by misstating the results of the MC\&A tasks. In the third variation, the facility does not know about any malicious insider activity, but an operational change is made to remove the dependency relationship among these activities - instead of one person performing both activities, two people perform these activities. The person who performs activity 1 is still assumed to be the malicious insider, and activity 4 is assumed to have the high level of dependence, the same as for the baseline set of activities because a single person (but not the malicious insider) always performs these tasks.

Tables 16 and 17 provide the detection timelines for the variations with the malicious insider and the insider mitigation, respectively. Figure 34 is a plot of these detection timelines. The original BHEPs for activities 1 and 4 provided in Table 13 for the set of MC\&A activities no longer apply. For the case of the malicious insider, these values in Table 16 are set to 1.0, as the insider who performs both these activities is trying to conceal malicious activity. The probability of detection for these individual activities is zero. Because activities 1 and 4 are the only ones performed on days 1 and 2, the daily probability of MC\&A detection is also zero. Over the ten-day timeline for this case MC\&A detection occurs only on days 3, 6 and 9 when an activity other than 1 or 4 is performed. Activity 3 is performed on these days and is defined to have a high level of dependence for its performance. For the case with malicious insider mitigation for activity 4, the daily BHEP values reflect the removal of the dependency between activity 


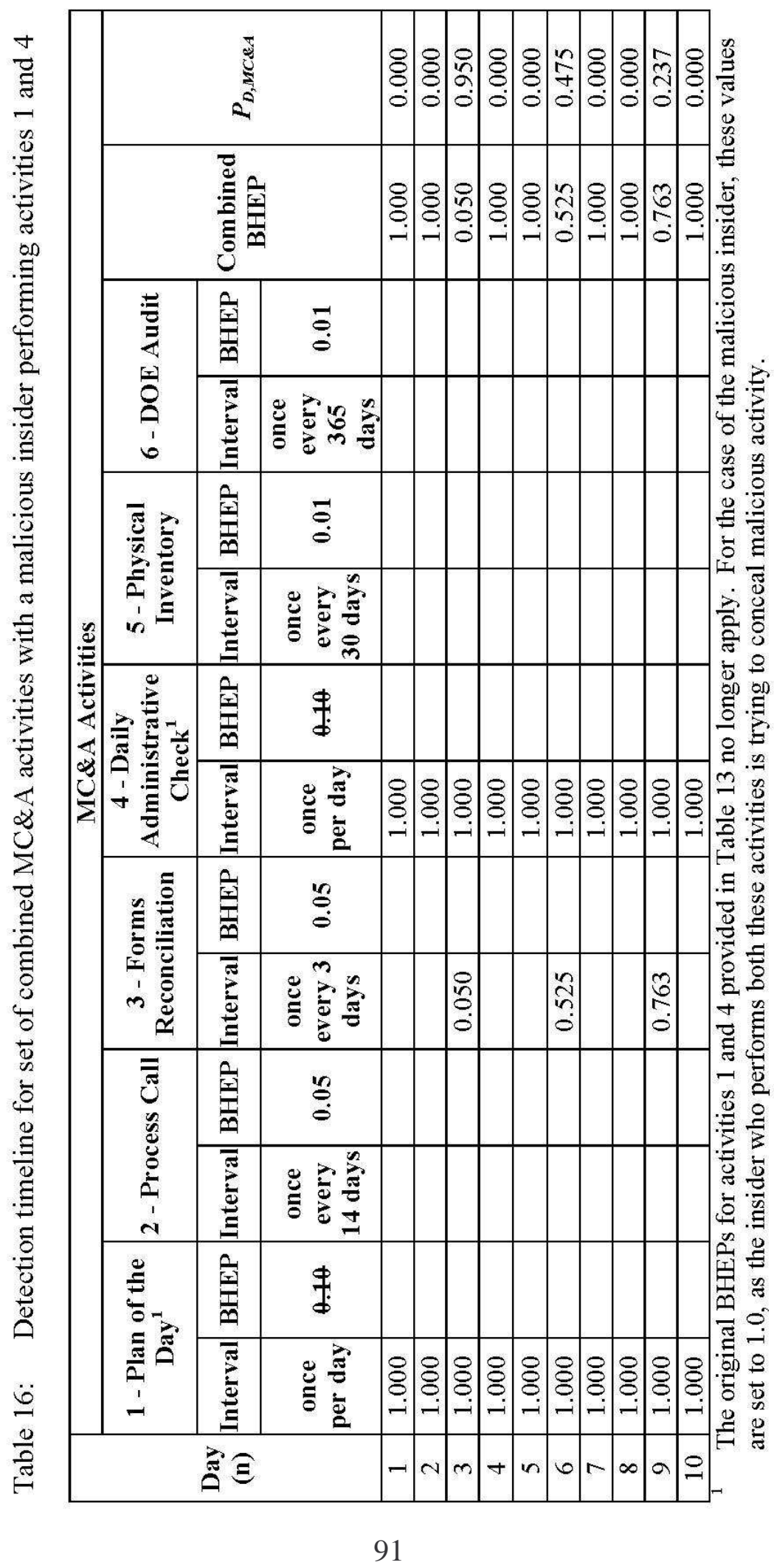




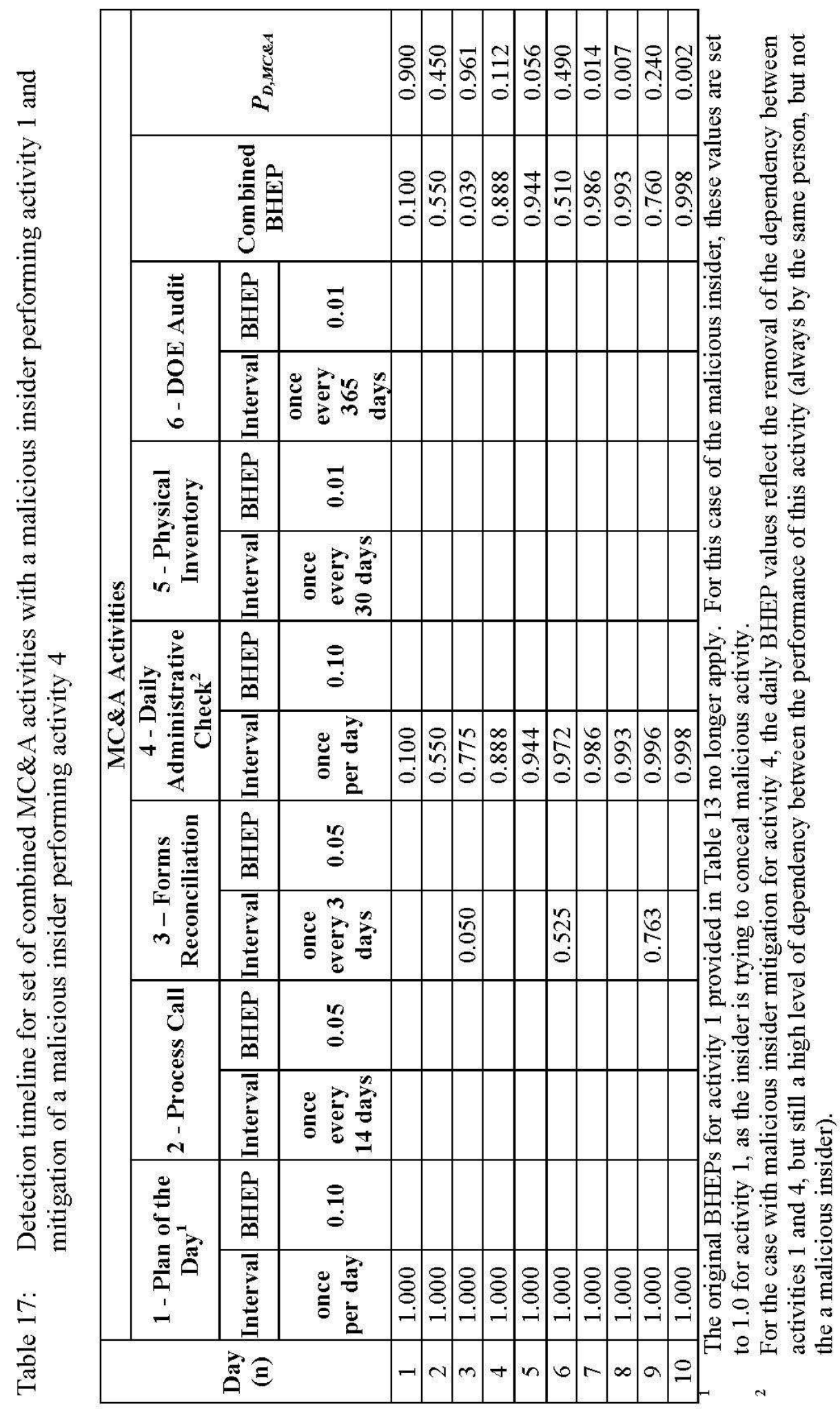




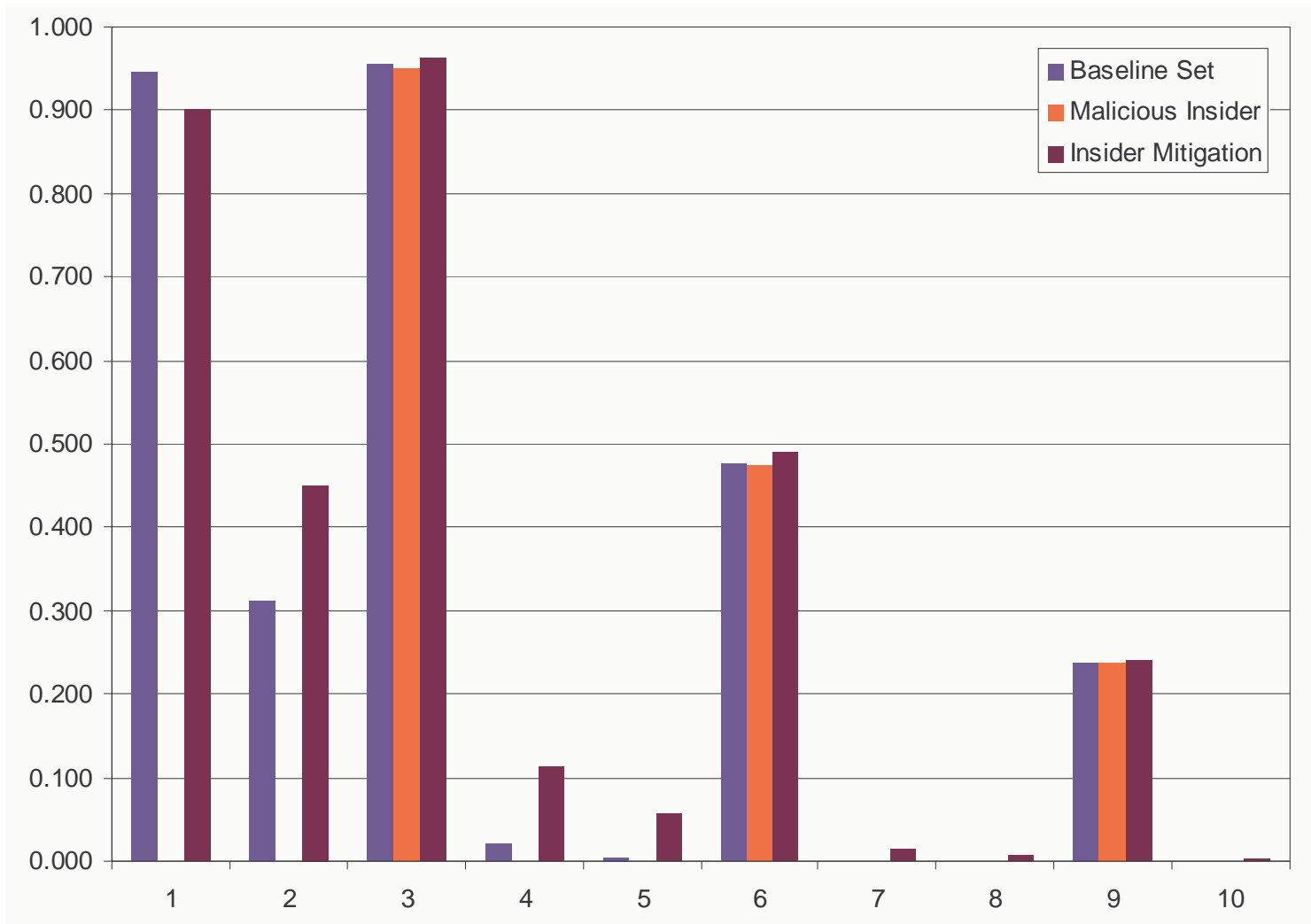

Figure 34: Detection timelines for baseline set of MC\&A activities, malicious insider, and insider mitigation.

1 and activity 4, but there is still a high level of dependence for the performance of activity 4 because the same person (although not a malicious insider) always performs this task. The operational change to remove the dependence between activities 1 and 4 to mitigate possible malicious insider actions results in additional daily MC\&A detection that is at least as high as or higher than the baseline case.

Table 18 provides the values for timely MC\&A detection in the MAA and PA and the end state summaries for each of the three cases. These results show that the case for malicious insider mitigation allows overall detection to recover up to the baseline case. These analyses demonstrate the application of the extended path analysis methodology to evaluate the effectiveness of a set of MC\&A activities, to identify possible vulnerabilities 
and to provide insights for operational strategies to address possible malicious insider activity.

Table 18: Timely MC\&A detection in the MAA (Event 2) and the PA (Event 4) and end state summary for baseline set of MC\&A activities, malicious insider activity and insider mitigation

\begin{tabular}{|c|c|c|c|c|}
\hline \multirow{2}{*}{$\begin{array}{l}\text { 5-day MAA/5-day PA } \\
\text { timeline scenario with } \\
\text { uniform theft distributions }\end{array}$} & \multicolumn{2}{|c|}{$\begin{array}{c}\text { Timely MC\&A } \\
\text { Detection }\end{array}$} & \multicolumn{2}{|c|}{ End State Summary } \\
\hline & $\begin{array}{c}\text { MAA } \\
\text { Event } 2\end{array}$ & $\begin{array}{c}\text { PA } \\
\text { Event } 4\end{array}$ & End State & Probability \\
\hline \multirow{4}{*}{$\begin{array}{l}\text { MC\&A detection timeline for } \\
\text { baseline set of activities and } \\
\text { dependency relationships }\end{array}$} & \multirow{4}{*}{0.980} & \multirow{4}{*}{0.507} & Material Recovered & 0.746 \\
\hline & & & Alert & 0.245 \\
\hline & & & $\begin{array}{l}\text { Material Recovered } \\
+ \text { Alert }\end{array}$ & 0.991 \\
\hline & & & Material Lost & 0.009 \\
\hline \multirow{4}{*}{$\begin{array}{l}\text { MC\&A detection timeline } \\
\text { assuming malicious insider } \\
\text { for daily activities } 1 \text { and } 4 \\
\text { with high dependence } \\
\text { relationship }\end{array}$} & \multirow{4}{*}{0.570} & \multirow{4}{*}{0.641} & Material Recovered & 0.583 \\
\hline & & & Alert & 0.272 \\
\hline & & & $\begin{array}{l}\text { Material Recovered } \\
+ \text { Alert }\end{array}$ & 0.855 \\
\hline & & & Material Lost & 0.145 \\
\hline \multirow{4}{*}{$\begin{array}{l}\text { MC\&A detection timelines } \\
\text { assuming insider mitigation } \\
\text { for activity } 4\end{array}$} & \multirow{4}{*}{0.968} & \multirow{4}{*}{0.699} & Material Recovered & 0.743 \\
\hline & & & Alert & 0.248 \\
\hline & & & $\begin{array}{l}\text { Material Recovered } \\
+ \text { Alert }\end{array}$ & 0.991 \\
\hline & & & Material Lost & 0.009 \\
\hline
\end{tabular}

\subsection{SUMMARY OF METHODS DEVELOPMENT FOR DAILY AND COMBINED MC\&A Detection AND MulTiPle PHySiCAl ProteCtion LAYERS}

The analyses presented in this chapter further demonstrate the use of the extended path analysis to model insider theft and integrated PPS and MC\&A protection elements and to quantify the effectiveness of these protection elements against an insider threat. The methods provide tools to evaluate the protection level MC\&A activities provide over time, identify gaps, and model potential insider activity. The results provide insights on how MC\&A activities can be implemented in facility operations to provide a desired level of protection over time. 


\section{Chapter 5: Conclusions and Recommendations}

The goal of this research was to develop a probabilistic basis and a new method to incorporate MC\&A protection elements explicitly within the existing probabilistic path analysis methodology to address insider theft. To accomplish this, three problem areas were addressed:

- "Detection" capabilities of MC\&A protections and quantitative probabilities of detection - individually, in combination, and as a function of time;

- Competing delay and detection timelines for insider theft versus facility detection; and

- Scenario development to integrate the evaluation of PPS and MC\&A protections within physical protection layers.

This work applied PRA methods to develop and demonstrate three key methods for incorporating MC\&A protection elements in to the existing probabilistic path analysis methodology, as follows:

1. HRA methods and HEPs for human performance of NPP operations to develop detection probabilities for MC\&A activities;

2. An object-based state paradigm to model the stages and timing for insider theft and to characterize insider theft as a race against detection by facility MC\&A activities; and

3. ESDs to incorporate MC\&A activities within the protection layers of a PPS, to develop insider theft scenarios, and to propagate detection probabilities for a theft scenario.

Using these approaches to characterize and evaluate MC\&A activities has demonstrated the importance of these activities as protection elements for insider theft. 
The application of HRA methods to define MC\&A detection probabilities also identified three key factors for "designing” MC\&A activities to address insider theft scenarios: (1) the type of operation based on a desired probability of detection, (2) the level of dependence in the performance of the operation, and (3) the scenario timelines of interest for achieving timely detection. While MC\&A activities do not indicate actual detection of an insider adversary, the timely detection afforded by MC\&A activities provides an alert that material is not where it should be. The possible end states (Material Recovered, Alert, and Material Lost) for each theft scenario provide additional insights about the status and recovery of critical assets. The "Material Recovered" end state indicates that an MC\&A activity alerted the facility that material was not where it should be and that subsequent actions recovered the material before it could be taken out of the facility. The "Material Lost" end state indicates that no MC\&A alert occurred, and the facility has no information that material is missing even at the end of the scenario timeline.

The demonstration analysis provides calculations for a range of initial MC\&A detection probabilities and several scenarios with different theft and MC\&A detection timelines. These calculations indicate that the methods developed in this work provide flexibility for application to a wide range of insider theft scenarios. In evaluating the results of the analysis, however, it is evident that these methods are likely to be most applicable for discontinuous timeline and protracted theft scenarios. Current methods are adequate for abrupt theft scenarios because these scenarios assume detection occurs almost immediately and thus can be analyzed using the exit path for an outside adversary attack.

The methods resulting from this work have been developed within the framework of the existing path analysis methodology, and as such can be integrated with existing 
methods and tools in a fairly straightforward manner. Additional work in the following areas will be required to accomplish this fully.

- Explore other approaches for developing insider adversary timelines and develop more concrete guidance for this part of the method.

- Develop a comprehensive MC\&A performance database to identify all possible MC\&A activities and the corresponding BHEPs, similar to the performance database that has been developed for PPS protection elements. Explore complementary aspects of the SFPI [20-22], Markov chain [53-56], and MSET [58-62] approaches that might provide the basis for performance data. The database would include the initial probabilities of detection, and would relate these activities to insider positions and their associated performance, as well as access, knowledge and authority. Development of an MC\&A performance data base will facilitate automation of this method in a software tool.

- Investigate the application of other HRA techniques in this methodology (e.g., Swain [64] and the NRC multidisciplinary framework [65]). These techniques could support more detailed characterization of some MC\&A activities to determine detection probabilities, as well as the development of the MC\&A performance data base. In addition, other HRA methods identify "error forcing contexts" and consider errors of omission as well as errors of commission for human operators. An insider who could create an error forcing context in an area of facility operations may be able to establish as system vulnerability that would facilitate a theft or diversion path.

- Develop metrics to be applied with the extended path analysis methodology to show the relative importance of particular MC\&A activities to preventing different types of insider theft scenarios. The method has provided significant 
insight for characterizing and evaluating a specific MC\&A program against specific theft scenarios. Importance metrics would extend these insights to allow analysts to better understand which MC\&A operations are useful and which may be an added burden or expense and support decision making to improve efficiency and save money.

- Analyze additional types of systems using this method to determine if more design heuristics for MC\&A systems could be identified.

- Use Monte Carle discrete event simulation methods to directly solve the problem for more complex timelines or MC\&A inspection regimes.

- Incorporate this method into a tool like ASSESS or ATLAS to automate insider path identification and link those paths to the generation of a discontinuous timeline and MC\&A activities in the performance data base to form more realistic estimates of $\mathrm{P}_{\mathrm{E}}$ without hand-crafting every scenario. Automated evaluation may also require linking with LHS so that the analyst has access to several types of probability distributions with which to represent the adversary timeline.

The methods developed in this work support the probabilistic basis for and have enabled the development of an extended path analysis methodology in which MC\&A protections can be combined with traditional sensor data in the calculation of PPS effectiveness. Explicitly incorporating MC\&A protection into the existing S\&S system evaluation provides a basis to measure the effectiveness measure of the PPS against insider threats. The resulting $\mathrm{P}_{\mathrm{E}}$ calculations will provide an integrated effectiveness measure that addresses both outsider and insider threats. 


\section{Appendix A: Combining Probability Distributions, Analytic, or Continuous Variable Case ${ }^{3}$}

Let $\mathrm{x}$ and $\mathrm{y}$ be independent variables having the probability density functions $\mathrm{p}_{\mathrm{x}}(\mathrm{x}), \mathrm{p}_{\mathrm{y}}(\mathrm{y})$. If $\mathrm{z}=\mathrm{x}+\mathrm{y}$, then the density function for $\mathrm{z}$ is expressed by the convolution integral

$$
p_{z}(z)=\int_{-\infty}^{\infty} p_{x}(x) p_{y}(z-x) d x
$$

Similarly, if

$$
z=x y
$$

then

$$
p_{z}(z)=\int_{-\infty}^{\infty} p_{x}(x) p_{y}\left(\frac{z}{x}\right) \frac{1}{x} d x
$$

(with any ambiguity at $\mathrm{x}=0$ handled by limit operations from both sides in the obvious way).

More generally, let

$$
z=f(x, y)
$$

where, for any specific values of $\mathrm{z}$ and $\mathrm{x}, \mathrm{y}$ has a specific value denoted by

$$
y=f^{-1}(z, x)
$$

that is

$$
z \equiv f\left[\left(x, f^{-1}(z, x)\right)\right]
$$

Then

$$
p_{z}(z)=\int p_{x}(x) p_{y}\left[f^{-1}(z, x)\right] \frac{\partial}{\partial z} f^{-1}(z, x) d x
$$

which may be thought of as a more general form of convolution. Again, there are obvious further generalizations possible but this is sufficient for our purposes.

In real life applied work, we rarely have the luxury of dealing with analytic forms and even in those rare cases may be unable to perform the integrations [Equation (A.3.8)] analytically. We are therefore led to seek approximate procedures.

3 The content here is an excerpt of Section A.3.1 from “Appendix A. PRA Methodology Detail” [47]. The equation numbers cited here correspond to the equation number from this reference 


\section{References}

[1] U.S. Department of Energy. 2006. Material Control and Accountability, DOE M 470.4-6. Washington DC: U.S. Department of Energy.

[2] Venkatesh, S., and C. Key. 2009. "A Self-Assessment of the Material Control and Accountability Implementation Function by National Nuclear Security Administration Headquarters (NNSA-HQ) Program Organization," in Proceedings of the $50^{\text {th }}$ Annual Meeting of the Institute for Nuclear Materials Management. Deerfield IL: Institute of Nuclear Materials Management.

[3] U.S. Department of Energy. 2005. Personnel Security, DOE M 470.4-5. Washington DC: U.S. Department of Energy.

[4] U.S. Department of Energy. 2009. Information Security, DOE M 470.4-4A. Washington DC: U.S. Department of Energy.

[5] Morzinski, J., and P. Dawson. 2000. "Designing Safeguards Performance Analysis to Determine and Validate Detection Probabilities, "Proceedings of the $41^{\text {st }}$ Annual Meeting of the Institute for Nuclear Materials Management. Deerfield IL: Institute of Nuclear Materials Management.

[6] Dawson, P.G., and P. Hester, 2006. "Real-Time Effectiveness Approach to Protecting Nuclear Materials," Proceedings of the $47^{\text {th }}$ Annual Meeting of the Institute for Nuclear Materials Management. Deerfield IL: Institute of Nuclear Materials Management.

[7] Dawson, P.G., P. Hester, T. Suski, and C. Williams. 2007. Real-Time Effectiveness Approach to Protecting Nuclear Materials, LCP SNL-1709, Unclassified Controlled Nuclear Information. Albuquerque NM: Sandia National Laboratories.

[8] Sandia National Laboratories. 2005. Department of Energy Office of Security and Safety Performance Assurance, Technology Transfer Manual, Vulnerability Assessment, SAND05-3929P, Unclassified Controlled Nuclear Information. Albuquerque NM: Sandia National Laboratories.

[9] International Atomic Energy Agency (IAEA). 1999. The Physical Protection of Nuclear Materials and Nuclear Facilities, IAEA-INFCIRC/225/Rev. 4 (Corrected). Vienna: IAEA.

[10] U.S. Dept. of the Army. 2001. Physical Security, Report FM 3-19.30. 
[11] IAEA, 2002. Handbook on the physical protection of nuclear materials and facilities, IAEA-TECDOC-1276, Official Use Only. Vienna: IAEA.

[12] Jaeger, C.D. 2002, "Risk Assessment Methodology for Chemical Facilities," in Probabilistic Safety Assessment and Management PSAM6, E. J. Bonano, A. L. Camp, M. J. Majors, and R. A. Thompson (eds.), pp. 1471-1476. Oxford: Elsevier Science Ltd.

[13] Bashurov, V V., V.O., Filimonenkov, A.A. Yaroslavtsev, and Y.I. Churikov. 2004. "Evaluation of Risk of Security Failure of a Protected Object," Journal of Nuclear Materials Management, Vol. XXXIII, No. 1, pp. 31-35. Deerfield IL: Institute of Nuclear Materials Management.

[14] Kim, C., S. Kwak, and C-H. Chung. 2005. "A Simulation Methodology for the Evaluation of the Physical Protection Systems in Nuclear Power Plants,"

Transactions, Vol. 93, pp. 320-321. Lagrange Park IL: American Nuclear Society.

[15] Whitehead, D.W., C.S. Potter, and S.L. O'Connor. 2007. Nuclear Power Plant Security Assessment Technical Manual, SAND2007-5591. Albuquerque NM: Sandia National Laboratories.

[16] U.S. Department of Energy. 2005. "Safeguards and Security Program References,” DOE M 470.4-1.Washington DC: U.S. Department of Energy.

[17] Garcia, M.L. 2008. The Design and Evaluation of Physical Protection Systems Second Edition, Boston: Butterworth-Heinemann.

[18] Garcia, M.L. 2005. Vulnerability Assessment of Physical Protection Systems. Boston: Elsevier Butterworth-Heinemann.

[19] Cipiti, B.B. 2009. "Virtual Safeguards Testing for Process Monitoring and Advanced Materials Accountancy," in Proceedings of the $50^{\text {th }}$ Annual Meeting of the Institute for Nuclear Materials Management. Deerfield IL: Institute of Nuclear Materials Management.

[20] Poorbaugh, J. 2009. "Comprehensive Analysis of Safeguards Strategies (COMPASS) as a Program Effectiveness Indicator," in Proceedings of the $50^{\text {th }}$ Annual Meeting of the Institute for Nuclear Materials Management. Deerfield IL: Institute of Nuclear Materials Management.

[21] Whitworth, A. 2009. "Safeguards First Principles Initiative (SFPI)," in Proceedings of the $50^{\text {th }}$ Annual Meeting of the Institute for Nuclear Materials Management. Deerfield IL: Institute of Nuclear Materials Management. 
[22] Johnson, G. 2008. "Criteria for Determination of MC\&A System Effectiveness," in Proceedings of the $49^{\text {th }}$ Annual Meeting of the Institute for Nuclear Materials Management. Deerfield IL: Institute of Nuclear Materials Management.

[23] Cipiti, B.B., P.E. Rexroth, and N.L. Ricker. 2007, Safeguards Performance Modeling of a UREX+1a Reprocessing Plant, SAND2007-6586. Albuquerque NM: Sandia National Laboratories.

[24] Parker, R. 2007. Inventory of Safeguards Software, LA-UR-07-6991. Los Alamos NM: Los Alamos National Laboratory.

[25] Hines, J.W., and J. Bowling. 2004. "An Expert System for Long-Term Monitoring of Special Nuclear Materials," Journal of Nuclear Materials Management, Vol. XXXIII, No. 4, pp. 13-21. Deerfield IL: Institute of Nuclear Materials Management.

[26] Raeder, C.R., D. Farmer, H. Burns, B. Trivett, and L. Bowers. 2004. "The Local Area Nuclear Material Accountability Software, (LANMAS)," Journal of Nuclear Materials Management, Vol. XXXIII, No. 1, pp. 55-58. Deerfield IL: Institute of Nuclear Materials Management.

[27] Janssens-Maenhout, G., and L. Dechamp. 2004. "Process Monitoring Appropriate for Near-Real-Time Accountancy," Journal of Nuclear Materials Management, Vol. XXXII, No. 3, pp. 10-16. Deerfield IL: Institute of Nuclear Materials Management.

[28] Waddoups, I.G., 1996, "National and International Nuclear Material Monitoring," presented at The $12^{\text {th }}$ Annual Joint Government-Industry Security Technology Symposium \& Exhibition, June-17-20, Williamsburg, VA.

[29] Jaeger, C., and I. Waddoups. 1995. "Nuclear Material Control in the United States," presented at the Workshop on Physical Protection, September 11-14, Moscow, Russia.

[30] Rodriguez, C. A., and I. Waddoups. 1993. "Safeguards Experience with Materials Monitoring Systems," Journal of Nuclear Materials Management. Deerfield IL: Institute of Nuclear Materials Management.

[31] Waddoups, I.G., and J.A. Abbott. 1993. "Material Control Evaluation," in Proceedings of the $34^{\text {th }}$ Annual Meeting of the Institute for Nuclear Materials Management. Deerfield IL: Institute of Nuclear Materials Management.

[32] Sandia National Laboratories. 2006. Advanced Vulnerability Analysis Overview Course, SAND2006-2096P. Albuquerque NM: Sandia National Laboratories. 
[33] Bennett, H. A. 1977. The EASI Approach to Physical Security Evaluation, SAND76-0500. Albuquerque NM: Sandia National Laboratories.

[34] Sandia National Laboratories 1989. SAVI: Systematic Analysis of Vulnerability to Intrusion, Vol. 1 and 2, SAND89-0926. Albuquerque NM: Sandia National Laboratories.

[35] ASSESS (Analytical System and Software for Evaluating Safeguards and Security), Version 2.56, Copyright 1989-2003. Livermore CA: Lawrence Livermore National Laboratory.

[36] ATLAS (Adversary Time-Line Analysis System) Software, Version 4.2. Build 171, developed at Sandia National Laboratories for the U.S. Department of Energy.

[37] Mullen, S. A., J.J. Davidson, and H.B. Jones, Jr. 1980. Potential Threat to Licensed Nuclear Activities from Insiders (Insider Study), NUREG-0703. Washington DC: U.S. Nuclear Regulatory Commission.

[38] Sutton, R H. 1983. Insider Adversary Study for the Office of Safeguards and Security, U.S. Department of Energy, Final Report, IEAL-294. Washington DC: International Energy Associates Limited.

[39] Hoffman, B., C. Meyer, B. Schwarz, and J. Duncan. 1990. Insider Crime: The Threat to Nuclear Facilities and Programs, R-3782-DOE, prepared for the U.S. Department of Energy. Santa Monica, CA: RAND Corporation.

[40] Brackney, R.C., and R.H. Anderson. 2004. Understanding the Insider Threat, Proceedings of a March 2004 Workshop. Santa Monica, CA: RAND Corporation.

[41] U.S. Department of Defense. 2000. DoD Insider Threat Mitigation, Final Report of the Insider Threat Integrated Process Team.

[42] Goodwin, K.E., J.C. Schleter, and M.D.K. Maltese. 1978. Diversion Path Analysis Handbook, HCP/D6010-01/1. Washington DC: U.S. Department of Energy.

[43] Boozer, D.D., and D. Engi. 1977. Insider Safeguards Effectiveness Model (ISEM) Users Guide, SAND77-0043. Albuquerque NM: Sandia National Laboratories.

[44] Lawrence Livermore National Laboratory. 1984. Safeguards Evaluation Method Insider Threat, UCID-20145, Rev. 2. Livermore CA: University of California. 
[45] U.S. Nuclear Regulatory Commission. 1975. Reactor Safety Study - An Assessment of Accident Risks in U.S. Commercial Nuclear Power Plants, WASH-1400 (NUREG-75/014). Washington DC: U.S. Nuclear Regulatory Commission.

[46] Breeding, R.J., J.C. Helton, E.D. Gorham, and F.T. Harper. 1992. "Summary Description of the Methods Using in the Probabilistic Risk Assessment for NUREG-1150," Nuclear Engineering and Design 135, pp. 1-27. Amsterdam: North Holland.

[47] Houston Lighting and Power Company. 1989. South Texas Project Probabilistic Safety Assessment, PLG-0675. Houston TX: Houston Lighting and Power Company.

[48] U.S. Nuclear Regulatory Commission. 1995. "Use of Probabilistic Risk Assessment Methods in Nuclear Activities: Final Policy Statement," Federal Register, Vol. 60, p. 42622, August 16.

[49] Jaeger, C.D. 2002. "Risk Assessment Methodology for Chemical Facilities," in Probabilistic Safety Assessment and Management PSAM6, E. J. Bonano, A. L. Camp, M. J. Majors, and R. A. Thompson (eds.), pp. 1471-1476. Oxford: Elsevier Science Ltd.

[50] Lockheed Martin Missiles \& Space. 1997. GPHS-RTGs in Support of the Cassini Mission Final Safety Analysis Report (FSAR). Philadelphia PA: Lockheed Martin.

[51] Miller, D. and J. Forester. 2000. Aviation Safety Human Reliability Analysis Method (ASHRAM), SAND2000-2955. Albuquerque NM: Sandia National Laboratories.

[52] Wheeler, T.A., K. Gawande, K. and S. Bespalko. 1997. "Development of RiskBased Ranking Measures of Effectiveness for the United States Coast Guard's Vessel Inspection Program," Risk Analysis, Vol. 17, No. 3. Society for Risk Analysis.

[53] Yue, M., L. Cheng, and R. Bari. 2005. Application of Probabilistic Methods to Proliferation Resistance: Misuse, Diversion, and Abrogation Scenarios. Upton NY: Brookhaven National Laboratory.

[54] Yue, M., L. Cheng, I. Papazoglou, M. Azarm, and R. A. Bari. 2005. "Calculations of Proliferation Resistance for Generation III Nuclear Energy Systems," Proceedings of Global 2005 International Conference on Nuclear Energy System for Future Generation and Global Sustainability, Tsukuba Japan. 
[55] Yue, M., L. Cheng, and R. A. Bari. 2008. "A Markov Model Approach to Proliferation-Resistance Assessment of Nuclear Energy Systems," Nuclear Technology, Vol. 162, pp. 26-44.

[56] Yue, M., L. Cheng, and R. A. Bari. 2009. "Relative Proliferation Risks for Different Fuel Cycle Arrangements," Nuclear Technology, Vol. 165, pp. 1-17.

[57] "Evaluation Methodology for Proliferation Resistance and Physical Protection of Generation IV Nuclear Energy Systems." 2006. Proliferation Resistance and Physical Protection Evaluation Method Experts Group, available on the Internet at http://www.gen-4.org/Technology/horizontal/PRPPEM.pdf.

[58] Sviridov, A.S., A.A. Petrov, I.N. Sazonov ,V.V. Erastov, A.V. Stepashko, A.A. Voronkov, B. Jensen, R. Elwood, L. Neymotin, and C. Roche. 2009. "Application of MSET Method for Assessing Effectiveness of the Material Control \& Accounting System at a Nuclear Facility," in Proceedings of the $50^{\text {th }}$ Annual Meeting of the Institute for Nuclear Materials Management. Deerfield IL: Institute of Nuclear Materials Management.

[59] Elwood, R., R. Brown, B.J. Campbell, C. Duncan, G.M. Fuller, G. Hammond, D. Hyde, B. Jensen, E. Owings, W. Brunsdon, M. Fontana, W. Kenna, G. Klopp, L. Neymotin, and C. Roche. 2008. "Benchmarking MSET: A Progress Report On The MC\&A System Effectiveness Tool," in Proceedings of the $49^{\text {th }}$ Annual Meeting of the Institute for Nuclear Materials Management. Deerfield IL: Institute of Nuclear Materials Management.

[60] Meppen, B., T. Bean, R. Haga, K. Moedl, J. Sanders, M.A. Thom. 2008. "Validation of Nuclear Material Control and Accountability (MC\&A) System Effectiveness Tool (MSET) at Idaho National Laboratory (INL)," in Proceedings of the $49^{\text {th }}$ Annual Meeting of the Institute for Nuclear Materials Management. Deerfield IL: Institute of Nuclear Materials Management.

[61] Elwood, R., B.J. Campbell, G.M. Fuller, G. Hammond, D. Hyde, B. Jensen, E. Owings, W. Brunsdon, M. Fontana, W. Kenna, G. Klopp, and C. Roche. 2007. "Nuclear Material Control And Accountability (MC\&A) Functional Model And MC\&A System Effectiveness Tool (MSET)," in Proceedings of the $48^{\text {th }}$ Annual Meeting of the Institute for Nuclear Materials Management. Deerfield IL: Institute of Nuclear Materials Management.

[62] Klopp, G.T., M. Fontana, C.T. Roche, W. Brunsdon, R.H. Elwood, B.A. Jensen, G.M. Fuller, B.J. Campbell, Hammond, E. Owings, W. Kenna. 2007. "The Use of Probabilistic Risk Assessment Technology for Evaluating MC\&A Effectiveness and Relative Risk Contributions," in Proceedings of the $48^{\text {th }}$ Annual Meeting of the Institute for Nuclear Materials Management. Deerfield IL: Institute of Nuclear Materials Management. 
[63] Wyss, G.D., and F.A. Durán. 2001. OBEST: The Object-Based Event Scenario Tree Methodology, SAND2001-0828. Albuquerque NM: Sandia National Laboratories.

[64] Swain III, A.D., and H.E. Guttmann. 1983. Handbook of Human Reliability Analysis with Emphasis on Nuclear Power Plants, SAND80-0200. Albuquerque NM: Sandia National Laboratories.

[65] Barriere, M.T., J.Wreathall, S.E. Cooper, D.C. Bley, W.J. Luckas, and A. RameySmith. 1995. Multidisciplinary Framework for Human Reliability Analysis with an Application to Errors of Commission and Dependencies, NUREG/CR-6526. Washington DC: U.S. Nuclear Regulatory Commission.

[66] Cooper, S.E., A.M. Ramey-Smith, J. Wreathall, G.W. Parry, D.C. Bley, W.J. Luckas, J.H. Taylor, and M.T. Barriere. 1996. A Technique for Human Error Analysis (ATHEANA), NUREG/CR-6350. Washington DC: U.S. Nuclear Regulatory Commission. 


\section{Vita}

Felicia Angelica Durán is a nuclear engineer in the Security Systems Analysis Department at Sandia National Laboratories (Sandia) in Albuquerque, New Mexico. She joined Sandia in September 1995 and was promoted to Principal Member of Technical Staff in August 2002. Felicia is a member of the Institute of Nuclear Materials Management (INMM), serves as Associated Editor for Physical Protection of the Journal of Nuclear Materials Management, and is on the Executive Committee of the Southwest Chapter of INMM. She earned her Masters Degree in Nuclear Engineering in December 1995 from The University of New Mexico (UNM) and was inducted into the UNM chapter of Tau Beta Pi, the National Engineering Honor Society. In May 1985, she received her Bachelors Degree in Materials Science and Engineering from the Massachusetts Institute of Technology (MIT), where she was an active student leader,

president of the Hispanic student group, a Charter Member of Alpha Phi (the first sorority at MIT) and an officer of the Intrafraternity Council, among other activities. She was awarded the MIT Karl Taylor Compton Award in 1985 for outstanding sustained community contribution.

Felicia has more than 20 years of professional experience supporting technical research programs for the U.S. Nuclear Regulatory Commission (NRC) and the U.S. Department of Energy (DOE). Most recent technical areas of experience include: integrated safeguards and security; security systems analysis; insider threat analysis; systems reliability and risk analysis; risk-informed regulation; methodology and software development for (1) nuclear reactor equipment condition monitoring and reliability, (2) object-based risk analysis methods with applications to aviation safety, and (3) dose modeling for decontamination and decommissioning; and technical project management. 
Other areas of experience include Level 2 probabilistic risk analysis (PRA) for boiling water reactors; environmental decision support; conceptual model development and treatment of uncertainty for performance assessment of waste management sites; expert judgement elicitation; regulatory compliance integration; waste management program support; geologic repository technology; nuclear waste transportation systems; emergency response training; and arms treaty verification.

She was born in Cuba, New Mexico, the oldest of four children. Her father was a coach with Cuba Schools and a coach, educator and middle school counselor with Albuquerque Public Schools in New Mexico. Her mother was a secretary for the Cuba Schools Superintendent, and for Longfellow, Mountain View and Dolores Gonzales Elementary Schools in Albuquerque. Felicia attended Armijo Elementary School and Ernie Pyle Middle School, and graduated Valedictorian of her class from Rio Grande High School in Albuquerque. She is the mother of a 14-year old daughter. Felicia has coached and plays soccer, and is an active community volunteer. She plays racquetball and has served on the local organizing committee for the World Senior Racquetball Championships since 1988. In 2003, she was one of four women nominated by Sandia for the Outstanding Women of Color in Research and Technology Awards; as one of the first 40 women to receive Emerald Honors, she was honored for Professional Achievement. In 2005, she received one of the Mayor Martin Chavez's first Move Up awards in recognition for her volunteer service in Albuquerque.

Permanent address: 11501 Paseo del Oso NE, Albuquerque, NM 87111

This dissertation was typed by the author. 